TRANSACTIONS OF THE

AMERICAN MATHEMATICAL SOCIETY

Volume 358, Number 7 , Pages 3133-3167

S 0002-9947(06)03845-1

Article electronically published on January 24, 2006

\title{
MAXIMAL FAMILIES OF GORENSTEIN ALGEBRAS
}

\author{
JAN O. KLEPPE
}

\begin{abstract}
The purpose of this paper is to study maximal irreducible families of Gorenstein quotients of a polynomial ring $R$. Let $\operatorname{GradAlg}^{H}(R)$ be the scheme parametrizing graded quotients of $R$ with Hilbert function $H$. We prove there is a close relationship between the irreducible components of $\operatorname{GradAlg}^{H}(R)$, whose general member is a Gorenstein codimension $(c+1)$ quotient, and the irreducible components of $\operatorname{GradAlg}^{H^{\prime}}(R)$, whose general member $B$ is a codimension $c$ Cohen-Macaulay algebra of Hilbert function $H^{\prime}$ related to $H$. If the Castelnuovo-Mumford regularity of the Gorenstein quotient is large compared to the Castelnuovo-Mumford regularity of $B$, this relationship actually determines a well-defined injective mapping from such "Cohen-Macaulay" components of GradAlg ${ }^{H^{\prime}}(R)$ to "Gorenstein" components of $\operatorname{GradAlg}^{H}(R)$, in which generically smooth components correspond. Moreover the dimension of the "Gorenstein" components is computed in terms of the dimension of the corresponding "Cohen-Macaulay" component and a sum of two invariants of $B$. Using linkage by a complete intersection we show how to compute these invariants. Linkage also turns out to be quite effective in verifying the assumptions which appear in a generalization of the main theorem.
\end{abstract}

\section{INTRODUCTION}

The main goal of this paper is to contribute to the classification of graded Gorenstein quotients of a polynomial ring $R$ of dimension $n+c$ over an algebraically closed field $k$. Let $\operatorname{GradAlg}^{H}(R)$ be the scheme parametrizing graded quotients $A$ of $R$ of $\operatorname{depth} A \geq \min (1, \operatorname{dim} A)$ and with Hilbert function $H$. We let $\mathrm{CM}_{c}^{H}(R)$ (resp. $\left.\operatorname{Gor}_{c}^{H}(R)\right)$ be the open subscheme parametrizing Cohen-Macaulay (resp. Gorenstein) quotients of codimension $c$ in $R$. In the Artinian case $\operatorname{Gor}_{c}^{H}(R)$ and the scheme PGor $(H)$ defined by certain catalecticant minors ([21]) are essentially the same (see Theorem 11). We call an irreducible component of $\operatorname{GradAlg}^{H}(R)$ which has non-empty intersection with $\mathrm{CM}_{c}^{H}(R)$ (resp. $\operatorname{Gor}_{c}^{H}(R)$ ) a "Cohen-Macaulay (resp. "Gorenstein) codimension $c$ " component. The main theorem of this paper establishes a strong connection between "Gorenstein codimension $(c+1)$ " components of $\operatorname{GradAlg}^{H}(R)$ and "Cohen-Macaulay codimension $c$ " components of $\operatorname{GradAlg}^{H^{\prime}}(R)$ for some $H^{\prime}$. Indeed the connection between these components allows us to deduce the generic smoothness and the dimension of the "Gorenstein

Received by the editors August 13, 2004.

2000 Mathematics Subject Classification. Primary 14C05, 13D10, 13D03, 13D07, 13C40.

Key words and phrases. Parametrization, Gorenstein algebra, Artinian algebra, liaison, licci, Cohen-Macaulay, canonical module, normal module, Hilbert scheme. 
codimension $(c+1)$ " component from the corresponding property and the number for the "Cohen-Macaulay codimension $c$ " component, and vica versa. If the socle degree of an Artinian reduction of the Gorenstein quotient is large enough (about twice the Castelnuovo-Mumford regularity, $\operatorname{reg}(B)$, of $B$ ), we then prove that this connection actually determines a well-defined injective mapping between the set of "Cohen-Macaulay codimension $c$ " components of $\operatorname{GradAlg}^{H^{\prime}}(R)$ and the set of "Gorenstein codimension $(c+1)$ " components of $\operatorname{GradAlg}^{H}(R)$ whose general members satisfy the Weak Lefschetz property (Theorem 24).

We all know the nice description of $\mathrm{CM}_{2}^{H}(R)$ given by the Hilbert-Burch theorem, and of $\operatorname{Gor}_{3}^{H}(R)$ proved by Buchsbaum and Eisenbud, in terms of the maximal minors (resp. the Pfaffians) of a suitable matrix. In this case the schemes $\mathrm{CM}_{2}^{H}(R)$ and $\operatorname{Gor}_{3}^{H}(R)$ are smooth and irreducible. There is, in our opinion, little hope of finding such a single nice description of $\operatorname{Gor}_{4}^{H}(R)$ in codimension 4, because this scheme has several irreducible components (3] and 22]). We think a more promising strategy should consist of classifying the irreducible components of $\mathrm{Gor}_{4}^{H}(R)$ together with describing the members of each family concretely. In this context the main theorems show that the corresponding classification of $\mathrm{CM}_{3}^{H^{\prime}}(R)$ is central for the classification of $\operatorname{Gor}_{4}^{H}(R)$, and they provide a method for answering Iarrobino and Srinivasan's question ([22]) about whether the non-irreducibility of $\operatorname{Gor}_{4}^{H}(R)$ is a rare phenomenon or not. In fact we give several classes of examples of reducible schemes $\operatorname{Gor}_{4}^{H}(R)$ whose general members are one-dimensional Gorenstein quotients of $R$ (Example 26 and Remark 27).

More precisely, let the graded algebra $A$ be a codimension one quotient of $B$, well defined by a twist of the canonical module $K_{B}$, by which we mean that there is a graded exact sequence

$$
0 \rightarrow K_{B}(-s) \rightarrow B \rightarrow A \rightarrow 0,
$$

where $B$ is assumed generically Gorenstein (see the beginning of Section 2). Let $K_{B}^{*}$ be the $B$-dual of the canonical module of $B=R / I_{B}$, let ${ }_{v} \operatorname{Ext}_{B}^{i}(-,-), i \geq 0$, be the graded piece of $\operatorname{Ext}_{B}^{i}(-,-)$ of degree $v$ and let ${ }_{v} \operatorname{ext}_{B}^{i}(-,-)$ be its dimension as a $k$-vectorspace. Then we show (cf. Theorem [16)

Theorem 1. Let $B=R / I_{B}$ be a graded Cohen-Macaulay quotient of a polynomial ring $R$, and let $A$ be a graded codimension one quotient of $B$, well defined by a twist of the canonical module $K_{B}$. and

A) If $B$ is licci, then $A$ is unobstructed as a graded $R$-algebra, $A$ is Gorenstein

$$
\operatorname{dim}_{(A)} \operatorname{GradAlg}^{H_{A}}(R)=\operatorname{dim}_{(B)} \operatorname{GradAlg}^{H_{B}}(R)+\operatorname{dim}\left(K_{B}^{*}\right)_{s}-1-\delta(B)_{-s},
$$

where

$$
\delta(B)_{-s}={ }_{-s} \operatorname{hom}_{B}\left(I_{B} / I_{B}^{2}, K_{B}\right)-{ }_{-s} \operatorname{ext}_{B}^{1}\left(I_{B} / I_{B}^{2}, K_{B}\right) .
$$

Moreover, if char $(k)=0$, then the codimension of the $H_{B}$-stratum of $A$ at $(B \rightarrow A)$ is $-\operatorname{ext}_{B}^{1}\left(I_{B} / I_{B}^{2}, K_{B}\right)$.

B) If $\operatorname{Proj}(B)$ is locally licci and $s>>0$, then $A$ is $H_{B}$-generic and Gorenstein, and

$$
\operatorname{dim}_{(A)} \operatorname{GradAlg}^{H_{A}}(R)=\operatorname{dim}_{(B)} \operatorname{GradAlg}^{H_{B}}(R)+\operatorname{dim}\left(K_{B}^{*}\right)_{s}-1 .
$$

Moreover, $A$ is unobstructed as a graded $R$-algebra if and only if $B$ is unobstructed as a graded $R$-algebra. 
If we assume $K_{B}(-s)_{v}=0$ for $v$ less than or equal to the largest degree of a minimal generator of $I_{B}$, then the codimension of the $H_{B}$-stratum of $A$ at $(B \rightarrow$ $A$ ), mentioned in Theorem [A), is just the codimension at $(A)$ of the stratum of $\operatorname{GradAlg}^{H_{A}}(R)$ of quotients $R \rightarrow A^{\prime}$ for which there exists some factorization $B^{\prime} \rightarrow A^{\prime}$ such that $B^{\prime}$ has Hilbert function $H_{B}$. Near $(A)$ this stratum turns out to be the stratum of quotients $A^{\prime}$ given by (10), with $A^{\prime}, B^{\prime}$ replacing $A, B$ in (1) (Lemma 7 and Proposition[13A). $A$ is $H_{B}$-generic if there is an open subset $U \ni(A)$ of $\operatorname{GradAlg}^{H_{A}}(R)$ contained in this stratum.

Note that if $B=R / I$ and $R / J$ are geometrically linked by a complete intersection, then $A=R /(I+J)$ is given by (1). In this case the unobstructedness of $A$ is known if $B$ is licci ([39]). It is standard to use sums of geometrically linked ideals to construct Gorenstein algebras (e.g. 8], 9], 10, 15]). By the corresponding more general construction of using geometric Gorenstein linkage, we also get Gorenstein algebras (e.g. [34; see also [35], Ex. 10.2). Since both constructions are given by (11) (e.g. 34], 30], p. 785), Theorem 1 applies in both cases.

In Theorem 14), $A$ is in fact shown to be unobstructed in the sense $\mathrm{H}^{2}(R, A, A)=$ 0 , a formula which also holds when $A$ is non-graded (Theorem 30). Moreover we prove a more general result that implies Theorem 1(Proposition 13). To use Proposition 13 we need to verify the vanishing of certain Ext-groups. These vanishings always hold in the licci case. We also prove that the scheme ZGor $(H)$ consisting of not necessarily graded Artinian Gorenstein quotients of $R$ is smooth at a graded quotient $(A)$ given by (11) provided $B$ is licci, and we give a formula for its dimension (Proposition 29). In the final Section 3 we show how we can use linkage to compute the mentioned Ext-groups as well as to find the other dimension invariants of Theorem 1 (Proposition 33 and Corollary 37, see Example 2 below).

In Theorem 1B), we may make " $s>>0$ " quite explicit. For instance if $\operatorname{Proj}(B)$ is a zero-dimensional scheme of length $d$, it may be replaced by " $s \geq 2 \operatorname{reg}\left(I_{B}\right)$ " (Remark 22, cf. (77)). In this case $\operatorname{dim}\left(K_{B}^{*}\right)_{s}=d$ and the $h$-vector of $A$ contains a consecutive subsequence of the form $(d, d, d)$. It is precisely in such ranges of $s$ that Theorem 24 applies, telling that there is a well-defined injective application $\pi$ from the set of irreducible components of $\operatorname{GradAlg}^{H_{B}}(R)$, whose general members satisfy the assumptions of Theorem $1 \mathrm{~B})$, to the set of irreducible "Gorenstein components" of $\operatorname{GradAlg}^{H_{A}}(R)$, whose general members satisfy the Weak Lefschetz property. This implies in particular that the $h$-vector of $A$ is an SI-sequence ([15]). In this range of $s$ one knows that any codimension one Gorenstein quotient is given by (11) (4]). Theorem 24 is a consequence of a thoroughly studied correspondence (Proposition 23). If the codimension of $B$ is $c=2$, then Theorem $1 \mathrm{~B}$ ), the application $\pi$ and the mentioned correspondence are well understood by Iarrobino-Kanev's results on "annihilating schemes", in which they construct the algebra $B$ from a given $A$ ([21, Ch. 5). Their approach applies also to $c \geq 3$ and leads e.g. to reducible $\operatorname{Gor}_{c+1}^{H}(R)$ for $c \geq 4$ ([21], Ch. 6) and to a dimension formula of $\operatorname{Gor}_{c+1}^{H}(R)$, provided the "first half" of $H$ coincides with the Hilbert function of a complete intersection (21], Thm. 4.17). The application $\pi$ is surjective for $c=2$, while Boij points out that such a surjectivity is not always true in higher codimensions ([3]). Also, results on the codimension of the Betti-strata can be related to the codimension statement in Theorem 11A) (see [21, Sect. 5.3.5 and Remark 25b)). Note that it is known that not every codimension 3 quotient is obtained by (11) ([36], [10]), i.e. that maximal families of quotients given by (10) may correspond to proper 
strata. This fits nicely with Theorem[A) in which the number $\left.{ }_{-s} \operatorname{ext}_{B}^{1}\left(I_{B} / I_{B}^{2}, K_{B}\right)\right)$ essentially measures the codimension of such strata.

In order to prove our results we study in full generality deformations of a quotient $A$ of $R$ which themselves are quotients of some deformation of $B$. First we recall and further develop some partially known results on the unobstructedness and the "family-dimension" of such a quotient $A$ of $R$ with fixed Hilbert function $H_{A}$ (Theorem [5). Here we neither assume $B$ to be Cohen-Macaulay nor $A$ to be Gorenstein. In this generality the deformation theory developed by Laudal is central (31]). Theorem 5 is important for the main application of this paper because it simultaneously treats the two cases of interest: when the general member of an irreducible family $R \rightarrow A$ factors via some deformation of $B \rightarrow A$ (i.e. $A$ is $H_{B}$-generic), and when it does not.

To illustrate some of our results we consider the following example. Here the Hilbert function, $H_{B}(v):=\operatorname{dim}_{k} B_{v}$, of $B$ is written as a vector $\left(H_{B}(0), H_{B}(1)\right.$, $\left.H_{B}(2), \ldots\right)$. If $A$ is Artinian, we call $\left(H_{A}(0), H_{A}(1), \ldots, H_{A}(s)\right)$ the $h$-vector of $A$, where $s$, the socle degree of $A$, is by definition the smallest number such that $H_{A}(v)=0$ for $v>s$.

Example 2. Let $B=R / I_{B}$ be a one-dimensional almost complete intersection obtained by linking a complete intersection (c.i.) $B^{\prime}$ of three hypersurfaces of degree $2,2,2$ to $B$ via a c.i. $D$ of three hypersurfaces of degree $3,3,3$. A standard mapping cone construction yields

$$
0 \rightarrow R(-7)^{3} \rightarrow R(-6)^{3} \oplus R(-5)^{3} \rightarrow R(-3)^{4} \rightarrow I_{B} \rightarrow 0 .
$$

Hence the Castelnuovo-Mumford regularity $\operatorname{reg}\left(I_{B}\right)=5$, and it is easy to see that $(1,4,10,16,19,19,19, \ldots)$ and $(1,4,7,8,8,8, \ldots)$ are the Hilbert functions of $B$ and $B^{\prime}$, respectively. Now $B$ is obviously licci, and Theorem 11 applies to any quotient $A$ given by (1). Using (10) and duality one shows $H_{A}(v)=H_{B}(v)+$ $H_{B}(s-v)-19$. It follows that $s$ is the socle degree of $A$ and that the $h$-vector of $A$ is the $(s+1)$-tuple $(1,4,7,7,4,1),(1,4,10,13,10,4,1),(1,4,10,16,16,10,4,1)$, $(1,4,10,16,19,16,10,4,1)$ and $(1,4,10,16,19 \ldots, 19,16,10,4,1)$ for $s=5,6,7,8$, and $\geq 9$, respectively. It is known ([27, Proposition 1.7 or Proposition 33 of this paper) how to compute $\operatorname{dim}\left(N_{B}\right)_{0}$, the dimension of the tangent space of $\operatorname{GradAlg}^{H_{B}}(R)$ at $(B)$, from $\operatorname{dim}\left(N_{B^{\prime}}\right)_{0}$. Indeed

$$
\operatorname{dim}\left(N_{B}\right)_{0}=\operatorname{dim}\left(N_{B^{\prime}}\right)_{0}+3 H_{B}(3)-3 H_{B^{\prime}}(3) .
$$

Since $N_{B^{\prime}} \simeq B^{\prime}(2)^{\oplus 3}$ we get $\operatorname{dim}\left(N_{B}\right)_{0}=45$. Moreover, due to Remark 22, we have $\delta(B)_{-s}=0$ for $s \geq 2 \operatorname{reg}\left(I_{B}\right)=10$ and $\operatorname{dim}\left(K_{B}^{*}\right)_{s}=19$ for $s \geq 2 \operatorname{reg}\left(I_{B}\right)-3=$ 7. Hence, for $s \geq 10$ we get that $A$ is unobstructed and $H_{B}$-generic and that $\operatorname{dim}_{(A)} \operatorname{GradAlg}^{H_{A}}(R)=\operatorname{dim}_{(A)} \operatorname{PGor}\left(H_{A}\right)=63$. Note that the conclusions so far only use Theorem $1 \mathrm{~B}$ ) because we may replace the assumption $s>>0$ of Theorem $1 \mathrm{~B})$ by $s \geq 2 \operatorname{reg}\left(I_{B}\right)$.

However, to deal with $s<10$ we need Theorem 14) and that $B$ is licci (or that the explicitly mentioned Ext-groups of Proposition $13 \mathrm{~A}$ ) vanish). We also have to compute the numbers $\operatorname{dim}\left(K_{B}^{*}\right)_{s}$ and $\delta(B)_{-s}$. To do so, we have a nice connection between these invariants using liaison. Indeed by Proposition 33

$$
\operatorname{dim}\left(K_{B}^{*}\right)_{v}=\operatorname{dim} B_{v-5}+3 \operatorname{dim}\left(I_{B / D}\right)_{v-2}-\delta\left(B^{\prime}\right)_{v-10}
$$

and symmetrically, $\delta(B)_{v-10}=\operatorname{dim} B_{v-5}^{\prime}+3 \operatorname{dim}\left(I_{B^{\prime} / D}\right)_{v-2}-\operatorname{dim}\left(K_{B^{\prime}}^{*}\right)_{v}$ for any $v$. Since $B^{\prime}$ is a c.i., we easily compute $\delta\left(B^{\prime}\right)_{v}$ and $\left(K_{B^{\prime}}^{*}\right)_{v}$. Indeed $K_{B^{\prime}} \simeq B^{\prime}(2)$ 
and $\delta\left(B^{\prime}\right)_{v}={ }_{v} \operatorname{hom}_{B^{\prime}}\left(I_{B^{\prime}} / I_{B^{\prime}}^{2}, K_{B^{\prime}}\right) \simeq \operatorname{dim}\left(K_{B^{\prime}}\right)_{v+2}^{\oplus 3}$. We get that $\operatorname{dim}\left(K_{B}^{*}\right)_{v}=$ $H_{B}(v-5)+3 H_{B^{\prime}}(v-5)-3 H_{B^{\prime}}(v-6)$, i.e. $\operatorname{dim}\left(K_{B}^{*}\right)_{s}=4,13,19,19, \ldots$ for $s=5,6,7,8, \ldots$, respectively. Correspondingly, $\delta(B)_{-s}=20,2,-4,-1,0,0, \ldots$ for $s=5,6,7,8,9,10, \ldots$, respectively. Hence

$$
\operatorname{dim}_{(A)} \operatorname{PGor}\left(H_{A}\right)=\operatorname{dim}_{(A)} \operatorname{GradAlg}^{H_{A}}(R)=28,55,67,64,63,63, \ldots,
$$

for $s=5,6,7,8,9,10, \ldots$, respectively. If $s \geq 7$ we see from the resolution of $I_{B}$ that ${ }_{-s} \operatorname{hom}_{B}\left(I_{B} / I_{B}^{2}, K_{B}\right)=0$, and hence that ${ }_{-s} \operatorname{ext}_{B}^{1}\left(I_{B} / I_{B}^{2}, K_{B}\right)=-\delta(B)_{-s}=$ $4,1,0$ for $s=7,8,9$, respectively. By Theorem 11A) the numbers 4,1,0 represent the codimensions of the strata of quotients $R \rightarrow A^{\prime}, H_{A^{\prime}}=H_{A}$, given by (10). It follows that these strata form a 63-dimensional irreducible family at $(A)$ for all $s \geq 7$, while the dimension of the corresponding irreducible component is $63+$ ${ }_{-s} \operatorname{ext}_{B}^{1}\left(I_{B} / I_{B}^{2}, K_{B}\right)$. That the conclusion of Theorem 1A) holds for $s=7,8$, shows the existence of a quotient $A$ with Hilbert function $H_{A}$ which is not given by (1) for some $B$ with Hilbert function $H_{B}$ ! For a complete analysis on any codimension 3 almost c.i. $B$, see Example 40 ,

In this work the author was inspired by joint work with Chris Peterson, where we constructed Gorenstein quotients of $R$ in various ways. We showed for instance that if $M$ is a maximal Cohen-Macaulay $B$-module of rank $r$ and $\bigwedge^{r} \widetilde{M} \simeq \widetilde{K}_{B}(s)$ as sheaves (in a large enough open set $U$ of $\operatorname{Proj}(B)$ ), then any regular section of $\widetilde{M}^{*}(s)$ defines a Gorenstein quotient $R \rightarrow A$ of codimension $r+c$ provided $\mathrm{H}_{*}^{0}\left(U, \bigwedge^{i} \widetilde{M}\right)$ for $2 \leq i \leq r / 2$ are maximally Cohen-Macaulay (cf. 30] for details). The idea of this paper is to look at the construction and to use deformation theory to vary every object and morphism in the construction as much as possible, in order to see how large the corresponding stratum in $\operatorname{GradAlg}^{H}(R)$ will be. This paper looks at the $r=1$ case because so far it is only when $r=1$ that the author is able to show his results also for Artinian Gorenstein rings. In a forthcoming paper the author generalizes the results of this paper considerably by considering deformations of regular sections of a maximal Cohen-Macaulay module $M$ of any rank, but, unfortunately, there we must partially restrict to positively dimensional Gorenstein algebras. We thank Olav A. Laudal, Roy Skjelnes and Johannes Kleppe for clarifying discussions. The author heartily thanks the referee for many valuable comments and questions.

For the convenience of the reader we include a section of preliminaries where we give some space to the theory of deformations. Indeed it is not always easy to find a nice reference for the results we use because treatments in the literature are often either too general and concern general cohomology groups of algebras, or too special, assuming certain groups are zero or very manageable.

1.1. Preliminaries. Let $B$ be an $n$-dimensional graded quotient of a polynomial $k$-algebra $R$ ( $k$ is algebraically closed) in $n+c$ variables (of degree 1 ), and let $M$ and $N$ be finitely generated graded $B$-modules. Let $\operatorname{depth}_{J} M$ denote the length of a maximal $M$-sequence in a homogeneous ideal $J$ and let depth $M=\operatorname{depth}_{\mathfrak{m}} M$, where $\mathfrak{m}$ is the irrelevant maximal ideal. Let $\mathrm{H}_{J}^{i}(-)$ be the right derived functor of the functor, $\Gamma_{J}(-)$, of sections with support in $\operatorname{Spec}(B / J)$. Recall that $\operatorname{depth}_{J} M=$ $\inf \left\{\operatorname{depth} M_{\wp} \mid \wp \supseteq J, \wp\right.$ graded $\}$ and that $\operatorname{depth}_{J} M \geq r$ iff $\mathrm{H}_{J}^{i}(M)=0$ for $i<r$; cf. [12] or [16]. 
If $Y=\operatorname{Proj}(B)$ and $Z$ is closed in $Y$ and $U=Y-Z$, we let $\mathrm{H}_{*}^{0}(U, \widetilde{M})=$ $\bigoplus_{v} \mathrm{H}^{0}(U, \widetilde{M}(v))$. Then we have an exact sequence

$$
0 \rightarrow \mathrm{H}_{I(Z)}^{0}(M) \rightarrow M \rightarrow \mathrm{H}_{*}^{0}(U, \widetilde{M}) \rightarrow \mathrm{H}_{I(Z)}^{1}(M) \rightarrow 0
$$

and isomorphisms $\mathrm{H}_{I(Z)}^{i}(M) \simeq \mathrm{H}_{*}^{i-1}(U, \widetilde{M})$ for $i \geq 2$. In the case $\operatorname{depth}_{I(Z)} N \geq$ 2 the graded $\operatorname{Ext}_{B}^{1}(M, N)$ injects into the corresponding global $\operatorname{Ext}_{\mathcal{O}_{U}}^{1}$-group of sheaves. Indeed we have in this case an exact sequence in degree zero ([12, exp. $\mathrm{VI})$

$$
\begin{aligned}
{ }_{0} \operatorname{Ext}_{B}^{1}(M, N) & \hookrightarrow \operatorname{Ext}_{\mathcal{O}_{U}}^{1}\left(\left.\widetilde{M}\right|_{U},\left.\widetilde{N}\right|_{U}\right) \rightarrow{ }_{0} \operatorname{Hom}_{B}\left(M, \mathrm{H}_{I(Z)}^{2}(N)\right) \\
& \rightarrow{ }_{0} \operatorname{Ext}_{B}^{2}(M, N) \rightarrow \operatorname{Ext}_{\mathcal{O}_{U}}^{2}\left(\left.\widetilde{M}\right|_{U},\left.\widetilde{N}\right|_{U}\right),
\end{aligned}
$$

where the form of the middle term comes from a spectral sequence also treated in 12 .

A Cohen-Macaulay (resp. maximal Cohen-Macaulay) $B$-module satisfies depth $M$ $=\operatorname{dim} M($ resp. $\operatorname{depth} M=\operatorname{dim} B)$ by definition, or equivalently, $\mathrm{H}_{\mathfrak{m}}^{i}(M)=0$ for $i<\operatorname{dim} M($ resp. $i<\operatorname{dim} B)$. If $B$ is Cohen-Macaulay and $K_{B}=\operatorname{Ext}_{R}^{c}(B, R(-n-c))$ is the canonical module of $B$, we know by Gorenstein duality that the $v$-graded piece of $\mathrm{H}_{\mathfrak{m}}^{i}(M)$ satisfies

$$
{ }_{v} \mathrm{H}_{\mathfrak{m}}^{i}(M) \simeq{ }_{-v} \operatorname{Ext}_{B}^{n-i}\left(M, K_{B}\right)^{\vee} .
$$

Recall that two graded quotients, $R / J$ and $R / J^{\prime}$, are said to be linked by a complete intersection if there exists a homogeneous complete intersection ideal $L$ such that $J=L: J^{\prime}$ and $J^{\prime}=L: J$ (with $L \subseteq J \cap J^{\prime}$ ). The relationship of being linked generates the equivalence relation, "linkage". $B=R / I_{B}$ is said to be licci (and hence Cohen-Macaulay) if it is in the linkage class of a complete intersection (cf. 34] for a survey). We define licci for a quotient of a regular local ring correspondingly. Moreover, $\operatorname{Proj}(B)$ is said to be locally licci if each of its local rings are licci.

Even though we in this paper try to avoid the algebraic (co)homology groups $\mathrm{H}_{2}(R, B, B)$ and $\mathrm{H}^{2}(R, B, B)$ and instead use the corresponding Tor- or Ext-groups, we will occasionally need them. We recall that the former group is given by an exact sequence

$$
0 \rightarrow \mathrm{H}_{2}(R, B, B) \rightarrow \mathrm{H}_{1} \rightarrow G_{1} \otimes_{R} B \rightarrow I_{B} / I_{B}^{2} \rightarrow 0,
$$

in which $G_{1}$ is $R$-free, $G_{1} \rightarrow I_{B}$ is surjective and minimal, and $\mathrm{H}_{1}=\mathrm{H}_{1}\left(I_{B}\right)$ is the degree-one Koszul homology of $I_{B}$ [38. An ideal $I_{B}$ of $R$ is called syzygetic if $\mathrm{H}_{2}\left(R, R / I_{B}, R / I_{B}\right)=0$. If $I_{B}$ is syzygetic, then $\left(I_{B}\right)_{\wp}$ is syzygetic for any prime ideal $\wp$ of $R$. $I_{B}$ is called generically syzygetic if $\left(I_{B}\right)_{\wp}$ is syzygetic for any graded prime $\wp$ of $A s s(B)$. We define genericity of other properties similarly. Using (44) one shows that if $R \rightarrow B$ is generically a complete intersection and $B$ is licci, then $\mathrm{H}_{2}(R, B, B)=0$ because $\mathrm{H}_{1}$ is a maximal Cohen-Macaulay module in the licci case $[20$. Thus, generically complete intersection licci ideals are syzygetic. For the graded group $\mathrm{H}^{2}(R, B, B)$ we remark that there is an exact sequence

$$
0 \rightarrow{ }_{0} \operatorname{Ext}_{B}^{1}\left(I_{B} / I_{B}^{2}, N\right) \rightarrow{ }_{0} \mathrm{H}^{2}(R, B, N) \rightarrow{ }_{0} \operatorname{Hom}_{B}\left(\mathrm{H}_{2}(R, B, B), N\right) \rightarrow .
$$

This comes from the spectral sequence $\operatorname{Ext}_{B}^{p}\left(\mathrm{H}_{q}(R, B, B), N\right)$, which converges to $\mathrm{H}^{p+q}(R, B, N)$ and $I_{B} / I_{B}^{2} \simeq \mathrm{H}_{1}(R, B, B)$; cf. [1], Prop. 16.1, or [31]. 
A Cohen-Macaulay quotient $B=R / I_{B}$ of codimension $c=\operatorname{dim} R-\operatorname{dim} B$ in $R$ has a minimal $R$-free resolution of the following form (cf. [7]):

$$
0 \rightarrow G_{c} \rightarrow \ldots \rightarrow G_{1} \rightarrow R \rightarrow B \rightarrow 0, \quad G_{j}=\bigoplus_{i=1}^{r_{j}} R\left(-n_{j, i}\right)
$$

In this case the Castelnuovo-Mumford regularity of $I_{B}$ is given by (cf. 34, p. 8)

$$
\operatorname{reg}\left(I_{B}\right)=\max _{j, i}\left\{n_{j, i}-j+1\right\}=\max _{i}\left\{n_{c, i}\right\}-c+1 .
$$

Let $G_{i}^{*}$ be the $R$-dual of $G_{i}$. Since $K_{B}=\operatorname{Ext}_{R}^{c}(B, R(-n-c))$, the $R$-dual complex of (6) yields a minimal $R$-free resolution of the twisted canonical module $K_{B}(n+c)$. In particular if $c=2$, it induces a complex

$$
0 \rightarrow N_{B} \rightarrow G_{1}^{*} \otimes_{R} B \rightarrow G_{2}^{*} \otimes_{R} B \rightarrow K_{B}(n+c) \rightarrow 0
$$

which is exact by e.g. 30, p. 795 .

Let $\operatorname{Hilb}^{p}(\mathbb{P})$ be Grothendieck's Hilbert scheme parametrizing closed subschemes $Y$ of $\mathbb{P}=\operatorname{Proj} R$ with Hilbert polynomial $p \in \mathbb{Q}[t]$; cf. [11. A $k$-point of $\operatorname{Hilb}^{p}(\mathbb{P})$ which corresponds to the subscheme $Y$ is usually denoted by $(Y \subset \mathbb{P})$ or just $(Y)$. A closed subscheme $Y$ of $\mathbb{P}$ is called unobstructed if $\operatorname{Hilb}^{p}(\mathbb{P})$ is smooth at $(Y \subset \mathbb{P})$.

Let $\operatorname{GradAlg}{ }^{H}:=\operatorname{GradAlg}^{H}(R)$ be the stratum of $\operatorname{Hilb}^{p}(\mathbb{P})$ given by deforming $Y \subset \mathbb{P}$ with constant Hilbert function $H_{Y}=H$ (i.e. its functor deforms both $Y \subset \mathbb{P}$ and its homogeneous coordinate ring, B, flatly); cf. [27. $\operatorname{GradAlg}^{H}(R)$ allows a natural scheme structure whose tangent (resp. "obstruction") space at $(Y \subset \mathbb{P})$ is ${ }_{0} \operatorname{Hom}_{B}\left(I_{B} / I_{B}^{2}, B\right) \simeq{ }_{0} \operatorname{Hom}_{R}\left(I_{B}, B\right) \quad\left(\right.$ resp. $\left.{ }_{0} \mathrm{H}^{2}(R, B, B)\right)$, i.e. it is given by deforming $B$ as a graded $R$-algebra [24. In the case when $H(v)$ does not vanish for large $v$ (i.e. $B$ is non-Artinian), we may look upon $\operatorname{GradAlg}^{H}(R)$ as parametrizing graded $R$-quotients, $R \rightarrow B$, satisfying $\operatorname{depth}_{\mathfrak{m}} B \geq 1$ and with Hilbert function $H_{B}=H$. If $B$ is Artinian, $\operatorname{GradAlg}^{H}(R)$ still represents a functor parametrizing graded $R$-quotients with Hilbert function $H_{B}=H$ (see Proposition 9), and its open subscheme of Gorenstein quotients coincides, at least topologically and infinitesimally, with PGor $(H)$ (the corresponding scheme of forms with "catalecticant structure"; see Theorem 111 below and the material before it). $B$ is called unobstructed as a graded $R$-algebra iff $\operatorname{GradAlg}^{H_{B}}(R)$ is smooth at $(R \rightarrow B)$. This definition of unobstructedness coincides with the one given in Remark 3 below by 31] or [37, p. 151. By [24, Remark 3.7,

$$
\operatorname{GradAlg}^{H}(R) \simeq \operatorname{Hilb}^{p}(\mathbb{P}) \text { at }(Y \subset \mathbb{P}),
$$

provided ${ }_{0} \operatorname{Hom}_{R}\left(I_{B}, \mathrm{H}_{\mathfrak{m}}^{1}(B)\right)=0$. This happens for quotients $B$ of $\operatorname{depth}_{\mathfrak{m}} B \geq 2$.

Similarly we let $\operatorname{Grad} \operatorname{Alg}\left(H_{B}, H_{A}\right)$ be the representing object of the functor deforming flags (surjections) $B \rightarrow A$ of graded quotients of $R$ of positive depth (for non-Artinian quotients) and with Hilbert functions $H_{B}$ and $H_{A}$ of $B$ and $A$, respectively. The tangent space ${ }_{0} A_{B \rightarrow A}^{1}$ of $\operatorname{GradAlg}\left(H_{B}, H_{A}\right)$ at $(B \rightarrow A)$ and the tangent maps $T_{p}$ and $T_{q}$ of the second projection $p: \operatorname{GradAlg}\left(H_{B}, H_{A}\right) \rightarrow$ $\operatorname{GradAlg}^{H_{A}}(R)$ (essentially given by $\left.p\left(\left(B^{\prime} \rightarrow A^{\prime}\right)\right)=\left(A^{\prime}\right)\right)$ and the first projection $q: \operatorname{GradAlg}\left(H_{B}, H_{A}\right) \rightarrow \operatorname{GradAlg}^{H_{B}}(R)$ are given by the cartesian square in the 
following diagram of exact sequences:

(10)

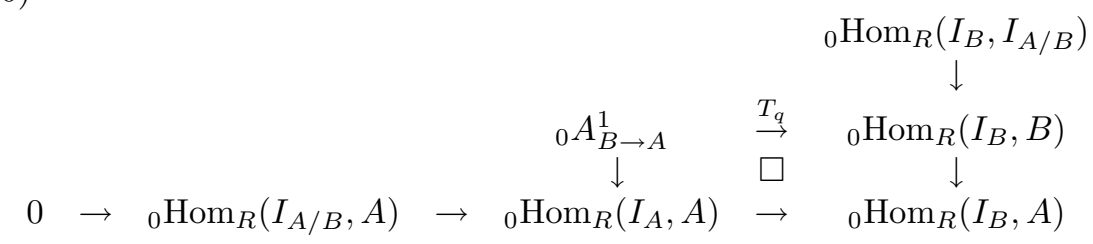

Here $B \rightarrow A \simeq R / I_{A}$ is a surjection of graded $R$-algebras with kernel $I_{A / B}$. Since the lower sequence in the diagram above may be continued by ${ }_{0} \mathrm{H}^{2}(B, A, A)$ to make a long exact sequence of algebra cohomology groups, and since ${ }_{0} \mathrm{H}^{2}(B, A, A)$ injects in general into ${ }_{0} \operatorname{Ext}_{B}^{1}\left(I_{A / B}, A\right)$ ([14], exp. VI), it follows that ${ }_{0} \operatorname{Hom}_{R}\left(I_{A}, A\right) \rightarrow$ ${ }_{0} \operatorname{Hom}_{R}\left(I_{B}, A\right)$ is surjective provided ${ }_{0} \operatorname{Ext}_{B}^{1}\left(I_{A / B}, A\right)=0$. One may also prove this surjectivity in a more elementary manner by dualizing the exact sequence $0 \rightarrow I_{B} \otimes_{R} A \simeq I_{B} / I_{A} I_{B} \rightarrow I_{A} \otimes_{R} B \rightarrow I_{A / B} \rightarrow 0$.

Remark 3. Let $R \rightarrow B$ be a graded quotient, and let $\left(T, \mathfrak{m}_{T}\right) \rightarrow\left(S, \mathfrak{m}_{S}\right)$ be a small Artinian surjection (i.e. of local Artinian $k$-algebras with residue fields $k$ whose kernel $\mathfrak{a}$ satisfies $\mathfrak{a} \cdot \mathfrak{m}_{T}=0$ ). A graded deformation $B_{S}$ of $B$ to $S$ is a graded $S$-flat quotient of $R \otimes_{k} S$ satisfying $B_{S} \otimes_{S} k \simeq B$. A graded deformation $B_{T}$ of $B_{S}$ to $T$ is $T$-flat and satisfies $B_{T} \otimes_{T} S \simeq B_{S}$.

A flag (i.e. a surjection) of graded quotients of $R, B \rightarrow A$, is said to be unobstructed if for every small Artinian surjection $T \rightarrow S$ and for every graded deformation $B_{S} \rightarrow A_{S}$ of $B \rightarrow A$ to $S$, there exists a graded deformation $B_{T} \rightarrow A_{T}$ (of $T$-flat quotients) reducing to $B_{S} \rightarrow A_{S}$ via $(-) \otimes_{T} S$. Similarly a quotient $B$ of $R$ is unobstructed if every graded deformation $B_{S}$ deforms further to $T$.

The obstruction, $o_{0}\left(B_{S}\right)$, to deforming $B_{S}$ to $T$ sits in ${ }_{0} \mathrm{H}^{2}(R, B, B) \otimes_{k} \mathfrak{a}$, and ${ }_{0} \mathrm{H}^{1}(R, B, B) \otimes_{k} \mathfrak{a}$ corresponds to the set of graded deformations (hence ${ }_{0} \operatorname{Hom}_{R}\left(I_{B}, B\right) \simeq{ }_{0} \mathrm{H}^{1}(R, B, B)$ is the tangent space of $\left.\operatorname{GradAlg}^{H_{B}}(R)\right)$. More precisely if we fix a graded deformation $B_{T}$ of $B_{S}$ to $T$, then $B_{T}$ defines a bijection from ${ }_{0} \mathrm{H}^{1}(R, B, B) \otimes_{k} \mathfrak{a}$ onto the set of graded deformations of $B_{S}$ to $T$, allowing us to interpret $B_{T}+\lambda$ as another graded deformation of $B_{S}$ to $T$ for every non-trivial $\lambda \in{ }_{0} \mathrm{H}^{1}(R, B, B) \otimes_{k} \mathfrak{a}$; cf. [31, Thm. 2.2.5, or [24], Thm. 1.5. Similarly the obstruction to deforming $B_{S} \rightarrow A_{S}$ to a given graded deformation $B_{T}$ of $B_{S}$ sits in ${ }_{0} \mathrm{H}^{2}(B, A, A) \otimes_{k} \mathfrak{a}$, and ${ }_{0} \mathrm{H}^{1}(B, A, A) \otimes_{k} \mathfrak{a} \simeq{ }_{0} \operatorname{Hom}_{B}\left(I_{A / B}, A\right) \otimes_{k} \mathfrak{a}$ corresponds to the set of such deformations. Note that, by definition, the vanishing of the obstruction is equivalent to the existence of the corresponding desired deformation. Hence the vanishing of the obstruction groups ("spaces") leads to the unobstructedness of the corresponding objects.

Finally let $\phi_{S}: B_{S} \rightarrow A_{S}$ be a graded deformation of $\phi: B \rightarrow A$ to $S$, and let $B_{T}$ and $A_{T}$ be given graded deformations of $B_{S}$ and $A_{S}$, respectively, to $T$. By [31, Thm. 2.3.3, or [24], Thm. 1.6, there is an obstruction $o_{0}\left(\phi_{S} ; B_{T}, A_{T}\right) \in$ ${ }_{0} \mathrm{H}^{1}(R, B, A) \otimes_{k} \mathfrak{a}$ which vanishes if and only if there exists a graded morphism $\phi_{T}: B_{T} \rightarrow A_{T}$ such that $\phi_{T} \otimes_{T} i d_{S}=\phi_{S}$. Looking closer to Laudal's proof of [31, Thm. 2.3.3, one may see

$$
o_{0}\left(\phi_{S} ; B_{T}^{\prime}, A_{T}\right)-o_{0}\left(\phi_{S} ; B_{T}, A_{T}\right)=\left(\phi_{*} \otimes i d_{\mathfrak{a}}\right)(\lambda),
$$

where $B_{T}+\lambda=B_{T}^{\prime}$ and $\phi_{*}$ is the morphism ${ }_{0} \mathrm{H}^{1}(R, B, B) \rightarrow{ }_{0} \mathrm{H}^{1}(R, B, A)$ induced by $\phi$. 
One may consider non-graded deformations of e.g. a quotient $B$ of $R$ in which case the whole algebra cohomology group $\operatorname{Hom}_{R}\left(I_{B}, B\right) \simeq \mathrm{H}^{1}(R, B, B$ ) (resp. $\left.\mathrm{H}^{2}(R, B, B)\right)$ serves as tangent (resp. "obstruction") space of the deformations of $B$ as an $R$-algebra. For graded deformations it is the degree zero piece of the cohomology groups which counts. For a generically complete intersection, $R \rightarrow B$ (or more generally, for a quotient $B$ which satisfies $\left.{ }_{0} \operatorname{Hom}_{B}\left(\mathrm{H}_{2}(R, B, B), B\right)=0\right)$, its obstructions sit in ${ }_{0} \operatorname{Ext}_{B}^{1}\left(I_{B} / I_{B}^{2}, B\right) \otimes \mathfrak{a}$ by (5).

The following proposition is a main result of the author's unpublished thesis. It (as well as the contents of (10) ) is just an application, with a few new ideas, of the general deep results of Laudal ([31] and 32, Sect. 2) on deformations of categories as briefly explained in [25], Sect. 2 and its appendix (see also [26], Sect. 1). Since the proofs of [25] or [26] are not in the generality we need in this paper, we include a proof.

Proposition 4. Let $B$ be a graded quotient of $R$ and let $\phi: B \rightarrow A$ be a graded surjection, inducing morphisms ${ }_{0} \operatorname{Hom}_{R}\left(I_{B}, B\right) \rightarrow{ }_{0} \operatorname{Hom}_{R}\left(I_{B}, A\right)$ and ${ }_{0} \mathrm{H}^{2}(R, B, B)$ $\rightarrow{ }_{0} \mathrm{H}^{2}(R, B, A)$.

(i) If ${ }_{0} \operatorname{Ext}_{B}^{1}\left(I_{A / B}, A\right)=0$ (or if ${ }_{0} \mathrm{H}^{2}(B, A, A)=0$ ), then the first projection $q$ : $\operatorname{GradAlg}\left(H_{B}, H_{A}\right) \rightarrow \operatorname{GradAlg}^{H_{B}}(R)$ is smooth with fiber dimension ${ }_{0} \operatorname{hom}_{B}\left(I_{A / B}, A\right)$ at $(B \rightarrow A)$.

(ii) If ${ }_{0} \operatorname{Hom}_{R}\left(I_{B}, B\right) \rightarrow{ }_{0} \operatorname{Hom}_{R}\left(I_{B}, A\right)$ is surjective and ${ }_{0} \mathrm{H}^{2}(R, B, B) \rightarrow$ ${ }_{0} \mathrm{H}^{2}(R, B, A)$ is injective, then the second projection $p: \operatorname{GradAlg}\left(H_{B}, H_{A}\right) \rightarrow$ $\operatorname{GradAlg}^{H_{A}}(R)$ is smooth with fiber dimension ${ }_{0} \operatorname{hom}_{R}\left(I_{B}, I_{A / B}\right)$ at $(B \rightarrow A)$.

(iii) If ${ }_{0} \operatorname{Hom}_{R}\left(I_{B}, B\right) \rightarrow{ }_{0} \operatorname{Hom}_{R}\left(I_{B}, A\right)$ is surjective and $B$ is unobstructed as a graded $R$-algebra, then $p$ is again smooth with fiber dimension ${ }_{0} \operatorname{hom}_{R}\left(I_{B}, I_{A / B}\right)$ at $(B \rightarrow A)$.

(iv) If both ${ }_{0} \operatorname{Hom}_{R}\left(I_{B}, B\right) \rightarrow{ }_{0} \operatorname{Hom}_{R}\left(I_{B}, A\right)$ and the connecting homomorphism ${ }_{0} \operatorname{Hom}_{R}\left(I_{B}, A\right) \rightarrow{ }_{0} \mathrm{H}^{2}(B, A, A)$ (cf. (10)) are surjective and if $B$ is unobstructed as a graded $R$-algebra, then $A$ is unobstructed as a graded $R$-algebra.

Proof. Using the definition of smoothness of a morphism (e.g. 24, p. 212) and the results of Remark 3, it is straightforward to get Proposition $4(\mathrm{i})$ from ${ }_{0} \mathrm{H}^{2}(B, A, A)$ $=0$ and the injection ${ }_{0} \mathrm{H}^{2}(B, A, A) \hookrightarrow{ }_{0} \operatorname{Ext}_{B}^{1}\left(I_{A / B}, A\right)$.

To see (ii) and (iii) let $\phi_{S}: B_{S} \rightarrow A_{S}$ be any graded deformation of $\phi$ to $S$, and let $A_{T}$ be a graded deformations of $A_{S}$ to $T$. By the definition of smoothness, it suffices to prove the existence of a graded deformation $B_{T}$ of $B_{S}$ to $T$ such that $o_{0}\left(\phi_{S} ; B_{T}, A_{T}\right)=0$. In (iii) the existence of some deformation $B_{T}^{\prime}$ of $B_{S}$ follows by assumption. In (ii) we utilize that the two morphisms ${ }_{0} \mathrm{H}^{2}(R, B, B) \otimes_{k} \mathfrak{a} \rightarrow$ ${ }_{0} \mathrm{H}^{2}(R, B, A) \otimes_{k} \mathfrak{a}$ and ${ }_{0} \mathrm{H}^{2}(R, A, A) \otimes_{k} \mathfrak{a} \rightarrow{ }_{0} \mathrm{H}^{2}(R, B, A) \otimes_{k} \mathfrak{a}$ induced by $\phi$ map the obstructions $o_{0}\left(B_{S}\right)$ and $o_{0}\left(A_{S}\right)$ onto the same element in ${ }_{0} \mathrm{H}^{2}(R, B, A) \otimes_{k} \mathfrak{a}$ by 31], proof of Thm. 4.1.14. Since $o_{0}\left(A_{S}\right)=0$ and the first morphism is injective by assumption, we get $o_{0}\left(B_{S}\right)=0$ and hence the existence of some deformation $B_{T}^{\prime}$ in this case as well. Now since $o_{0}\left(\phi_{S} ; B_{T}^{\prime}, A_{T}\right) \in{ }_{0} \mathrm{H}^{1}(R, B, A) \otimes_{k}$ $\mathfrak{a} \simeq \operatorname{Hom}_{R}\left(I_{B}, A\right) \otimes_{k} \mathfrak{a}$ and since $\phi_{*}$ is surjective, there is an element such that $\left(\phi_{*} \otimes i d_{\mathfrak{a}}\right)(\lambda)=o_{0}\left(\phi_{S} ; B_{T}^{\prime}, A_{T}\right)$. We get the smoothness of $p$ by the displayed formula of Remark 3 . Since smooth morphisms have smooth fibers, the fiber dimension is as claimed by [24], Thm. 1.6. 
Finally (iv) follows from (iii), Remark 3 and the fact that ${ }_{0} \mathrm{H}^{2}(B, A, A) \otimes_{k} \mathfrak{a} \rightarrow$ ${ }_{0} \mathrm{H}^{2}(R, A, A) \otimes_{k} \mathfrak{a}$ maps obstructions to obstructions (by 31, Cor. 4.1.15). We leave the details as an exercise since we do not fully need (iv) in this paper.

Let $\phi: B \rightarrow A$ be a graded surjection. Using Proposition 4, we get the following theorem which, at least in the geometric case and with various assumptions on $\operatorname{Proj}(B)$, is not new (25], Sect. 2, 26], Sect. 1, 29], Ch. 9, e.g. Thm. 9.4 and Prop. 9.14). Note that part B) of Theorem 5 has as a consequence that the generic member of an irreducible family of quotients $R \rightarrow A$ comes from a member of a family of quotients $R \rightarrow B \rightarrow A$. Since it is in general not true that a general member factors via a deformation of $B$ even though a special member does, in part A) of the theorem below we determine the codimension of the stratum of quotients which factors. To be precise, let $U \subset \operatorname{GradAlg}^{H_{A}}(R)$ be a sufficiently small open subset containing $(A)$. The $k$-points of the subset $p\left(p^{-1}(U)\right)$ of $U$ correspond to quotients $R \rightarrow A^{\prime}$ with Hilbert function $H_{A}$ for which there exists some factorization $B^{\prime} \rightarrow A^{\prime}$ such that $B^{\prime}$ has Hilbert function $H_{B}$. We will call $p\left(p^{-1}(U)\right)$ a stratum of $H_{B}$-factorizations around $(A)$, and $\operatorname{dim} U-\operatorname{dim} p\left(p^{-1}(U)\right)$ the codimension of the $H_{B}$-stratum of $A$. At least if $U$ is smooth, it is the ordinary codimension of $p\left(p^{-1}(U)\right)$ in $U$. $A$ is called $H_{B}$-generic if there is an open subset $U_{A}$ of $\operatorname{GradAlg}^{H_{A}}(R)$ such that $(A) \in U_{A} \subset p\left(p^{-1}(U)\right)$. The codimension of the $H_{B^{-}}$ stratum of $A$ at $(B \rightarrow A)$ is defined to be $\operatorname{dim} U-\operatorname{dim} p\left(U^{\prime}\right)$, where $U^{\prime} \subset p^{-1}(U)$ is the intersection of the irreducible components of $p^{-1}(U)$ which contains $(B \rightarrow A)$. Using small letters for the $k$-dimension of ${ }_{0} \operatorname{Hom}(-,-)$ and ${ }_{0} \operatorname{Ext}_{B}^{1}(-,-)$ we have

Theorem 5. Let $R$ be a graded polynomial $k$-algebra and let $B \simeq R / I_{B} \rightarrow A \simeq$ $B / I_{A / B}$ be a graded morphism of quotients of $R$ such that ${ }_{0} \operatorname{Ext}_{B}^{1}\left(I_{A / B}, A\right)=0$.

A) If ${ }_{0} \operatorname{Ext}_{B}^{1}\left(I_{B} / I_{B}^{2}, A\right)=0$ and $\left(I_{B}\right)_{\wp}$ is syzygetic for any graded prime $\wp$ of Ass $(A)$, then $A$ is unobstructed as a graded R-algebra. Moreover, if $\operatorname{depth}_{\mathfrak{m}} A \geq$ $\min (1, \operatorname{dim} A)$, then

$$
\begin{aligned}
\operatorname{dim}_{(A)} \operatorname{GradAlg}^{H_{A}}(R)= & { }_{0} \operatorname{hom}_{R}\left(I_{B}, B\right)+{ }_{0} \operatorname{hom}_{B}\left(I_{A / B}, A\right)-{ }_{0} \operatorname{hom}_{R}\left(I_{B}, I_{A / B}\right) \\
& +{ }_{0} \operatorname{ext}_{B}\left(I_{B} / I_{B}^{2}, I_{A / B}\right)-{ }_{0} \operatorname{ext}_{B}\left(I_{B} / I_{B}^{2}, B\right) .
\end{aligned}
$$

Furthermore let $B$ be unobstructed as a graded $R$-algebra and let $k$ be of characteristic zero. Then the codimension of the $H_{B}$-stratum of $A$ at $(B \rightarrow A)$ is

$$
{ }_{0} \operatorname{ext}_{B}^{1}\left(I_{B} / I_{B}^{2}, I_{A / B}\right)-{ }_{0} \operatorname{ext}_{B}^{1}\left(I_{B} / I_{B}^{2}, B\right)
$$

B) If ${ }_{0} \operatorname{Ext}_{B}^{1}\left(I_{B} / I_{B}^{2}, I_{A / B}\right)=0$ and $\left(I_{B}\right)_{\wp}$ is syzygetic for any graded prime $\wp$ of Ass $\left(I_{A / B}\right)$, then $A$ is $H_{B}$-generic. Moreover, $A$ is unobstructed as a graded $R$ algebra if and only if $B$ is unobstructed as a graded $R$-algebra. Indeed if $\operatorname{depth}_{\mathfrak{m}} A \geq$ $\min (1, \operatorname{dim} A)$ and $\operatorname{depth}_{\mathfrak{m}} B \geq \min (1, \operatorname{dim} B)$, then

$$
\begin{aligned}
& { }_{0} \operatorname{hom}_{R}\left(I_{A}, A\right)-\operatorname{dim}_{(A)} \operatorname{GradAlg}^{H_{A}}(R) \\
& \quad={ }_{0} \operatorname{hom}_{R}\left(I_{B}, B\right)-\operatorname{dim}_{(B)} \operatorname{GradAlg}^{H_{B}}(R),
\end{aligned}
$$

and

$$
\begin{aligned}
\operatorname{dim}_{(A)} \operatorname{GradAlg}^{H_{A}}(R)= & \operatorname{dim}_{(B)} \operatorname{GradAlg}^{H_{B}}(R) \\
& +{ }_{0} \operatorname{hom}_{B}\left(I_{A / B}, A\right)-{ }_{0} \operatorname{hom}_{R}\left(I_{B}, I_{A / B}\right) .
\end{aligned}
$$


Proof. A) By the definition of a syzygetic ideal we have $\mathrm{H}_{2}(R, B, B)_{\wp}=0$ for any $\wp$ of $A s s(A)$. It follows that ${ }_{0} \operatorname{Hom}_{B}\left(\mathrm{H}_{2}(R, B, B), A\right)=0$ and hence that ${ }_{0} \mathrm{H}^{2}(R, B, A)=0$ by (5). Since we have in general an injection ${ }_{0} \mathrm{H}^{2}(B, A, A) \hookrightarrow$ ${ }_{0} \operatorname{Ext}_{B}^{1}\left(I_{A / B}, A\right)\left(\left[14\right.\right.$, exp. VI), we get ${ }_{0} \mathrm{H}^{2}(B, A, A)=0$ by the general assumption of Theorem 5. It follows that ${ }_{0} \mathrm{H}^{2}(R, A, A)=0$ by the long exact sequence of algebra cohomology (e.g. [31, Thm. 3.3.4)

$$
\rightarrow{ }_{0} \mathrm{H}^{2}(B, A, A) \rightarrow{ }_{0} \mathrm{H}^{2}(R, A, A) \rightarrow{ }_{0} \mathrm{H}^{2}(R, B, A) \rightarrow .
$$

This proves the unobstructedness of $A$ and $\operatorname{dim}_{(A)} \operatorname{GradAlg}^{H_{A}}(R)={ }_{0} \operatorname{hom}_{R}\left(I_{A}, A\right)$. Now, looking to (10), we see that the vanishing of ${ }_{0} \operatorname{Ext}_{B}^{1}\left(I_{A / B}, A\right)$ translates to a certain surjectivity in the cartesian square of (10). Since the vertical sequence of Hom maps in (10) may be continued by

$$
\rightarrow{ }_{0} \operatorname{Ext}_{B}^{1}\left(I_{B} / I_{B}^{2}, I_{A / B}\right) \rightarrow{ }_{0} \operatorname{Ext}_{B}^{1}\left(I_{B} / I_{B}^{2}, B\right) \rightarrow{ }_{0} \operatorname{Ext}_{B}^{1}\left(I_{B} / I_{B}^{2}, A\right) \rightarrow,
$$

it is straightforward to get the dimension formula from the vanishing assumption of A).

To see that ${ }_{0} \operatorname{ext}_{B}^{1}\left(I_{B} / I_{B}^{2}, I_{A / B}\right)-{ }_{0} \operatorname{ext}_{B}^{1}\left(I_{B} / I_{B}^{2}, B\right)$ measures the codimension, we observe that $B \rightarrow A$ is unobstructed and hence that $\operatorname{dim}_{(B \rightarrow A)} \operatorname{Grad} \operatorname{Alg}\left(H_{A}, H_{B}\right)$ $=\operatorname{dim}{ }_{0} A_{B \rightarrow A}^{1}$ by Proposition 4 (i). It follows that

$$
p: \operatorname{GradAlg}\left(H_{B}, H_{A}\right) \rightarrow \operatorname{GradAlg}^{H_{A}}(R)
$$

is a morphism between smooth schemes (i.e. smooth at $(B \rightarrow A)$ and $(A)$, respectively) and with fiber dimension ${ }_{0} \operatorname{hom}_{B}\left(I_{B} / I_{B}^{2}, I_{A / B}\right)$, from which we may conclude by using the theorem of generic smoothness to $p$ at $(B \rightarrow A)$; cf. 29, first part of the proof of Prop. 9.14 for more details from a similar proof.

B) Since $\mathrm{H}_{2}(R, B, B)_{\wp}=0$ for any $\wp \in \operatorname{Ass}\left(I_{A / B}\right)$ by assumption, we get ${ }_{0} \mathrm{H}^{2}\left(R, B, I_{A / B}\right)=0$ from (5). By the long exact sequence of algebra cohomology associated to $0 \rightarrow I_{A / B} \rightarrow B \rightarrow A \rightarrow 0$, we get precisely that the assumptions of Proposition 4(ii) are satisfied. By Proposition 4 (i) and (ii), both projection morphisms $p$ and $q$ are smooth, and we deduce the $H_{B}$-genericity of $A$ and the equivalence of the unobstructedness of $A$ and $B$. Looking to (10) and the statement about the fiber dimensions of $p$ and $q$ in Proposition 4 (i) and (ii), we get the dimension formulas of Theorem [5A) as well.

Remark 6. a) Note that the proof of Theorem 5A) leads to ${ }_{0} \mathrm{H}^{2}(R, A, A)=0$ (and in fact to ${ }_{v} \mathrm{H}^{2}(R, A, A)=0$ by twisting the two $\operatorname{Ext}_{B}^{1}$ vanishing assumptions by $\left.v\right)$.

b) It is possible to weaken the assumption ${ }_{0} \operatorname{Ext}_{B}^{1}\left(I_{A / B}, A\right)=0$ in Theorem 5 to " ${ }_{0} \mathrm{H}^{2}(B, A, A)=0$ " and still reach the same conclusions as in Theorem [5 A) and $5 \mathrm{~B})$. One may also weaken " $\left(I_{B}\right)_{\wp}$ is syzygetic for any $\wp$ of $\operatorname{Ass}(A)$ " in part A) to " ${ }_{0} \operatorname{Hom}_{B}\left(\mathrm{H}_{2}(R, B, B), A\right)=0$ " and still reach the same conclusions as in Theorem [5A). Similarly we may in part B) replace " $\left(I_{B}\right)_{\wp}$ is syzygetic for any $\wp$ of $\operatorname{Ass}\left(I_{A / B}\right)$ " by " ${ }_{0} \operatorname{Hom}_{B}\left(\mathrm{H}_{2}(R, B, B), I_{A / B}\right)=0$ " or the entire assumption " ${ }_{0} \operatorname{Ext}_{B}^{1}\left(I_{B} / I_{B}^{2}, I_{A / B}\right)=0$ and $\left(I_{B}\right) \wp$ is syzygetic for any graded prime $\wp$ of $A s s\left(I_{A / B}\right)$ " by " ${ }_{0} \operatorname{Ext}_{B}^{1}\left(I_{B} / I_{B}^{2}, I_{A / B}\right) \rightarrow{ }_{0} \operatorname{Ext}_{B}^{1}\left(I_{B} / I_{B}^{2}, B\right)$ is injective, and $B$ is unobstructed as a graded $R$-algebra" and still get the conclusions of Theorem $5 \mathrm{~B}$ ). There is no real change in the proofs, except when $B$ is unobstructed, in which case we need to use Proposition 4 (iii) in the proof instead of Proposition 4(ii). These variations turn out to have an application, notably to the case that $A$ is Artinian. 
c) Continuing (12), we see that the condition ${ }_{0} \operatorname{Ext}_{B}^{1}\left(I_{B} / I_{B}^{2}, A\right)=0$ of part A) is satisfied provided that ${ }_{0} \operatorname{Ext}_{B}^{1}\left(I_{B} / I_{B}^{2}, B\right)=0$ and ${ }_{0} \operatorname{Ext}_{B}^{2}\left(I_{B} / I_{B}^{2}, I_{A / B}\right) \hookrightarrow$ ${ }_{0} \operatorname{Ext}_{B}^{2}\left(I_{B} / I_{B}^{2}, B\right)$ is injective.

By applying Theorem [5A) the codimension of the $H_{B}$-stratum of $A$ at $(B \rightarrow A)$ is often just the codimension of the $H_{B}$-stratum of $A$, due to

Lemma 7. Let $B \simeq R / I_{B} \rightarrow A \simeq B / I_{A / B}$ be a graded morphism of quotients of $R$ and let $m(B)$ be the largest degree of the minimal generators of $I_{B}$.

a) If $\left(I_{A / B}\right)_{v}=0$ for all $v \leq m(B)$, then ${ }_{0} \operatorname{Hom}_{R}\left(I_{B}, I_{A / B}\right)=0$ and $p^{-1}((A))$ consists of a single $k$-point (i.e. if $B^{\prime} \rightarrow A$ is a quotient such that $H_{B^{\prime}}=H_{B}$, then $B^{\prime}=B$ ).

b) If ${ }_{0} \operatorname{Ext}_{B}^{1}\left(I_{A / B}, A\right)=0$ (or if ${ }_{0} \mathrm{H}^{2}(B, A, A)=0$ ) and $\left(I_{A / B}\right)_{v}=0$ for all $v \leq m(B)$, then there is an open subset $U$ of $\operatorname{GradAlg}\left(H_{B}, H_{A}\right)$ containing $(B \rightarrow A)$ such that the restriction of the first projection $q: \operatorname{GradAlg}\left(H_{B}, H_{A}\right) \rightarrow$ $\operatorname{GradAlg}^{H_{B}}(R)$ to $U$ is smooth, and the restriction of the second projection $p$ to $U$ is unramified and injective. Moreover $U=p^{-1}(p(U))$. In particular if $B \rightarrow A$ satisfies the assumptions of Theorem [5A) (except for char $(k)=0$ ), then the codimension of the $H_{B}$-stratum of $A$ is

$$
{ }_{0} \operatorname{ext}_{B}^{1}\left(I_{B} / I_{B}^{2}, I_{A / B}\right)-{ }_{0} \operatorname{ext}_{B}^{1}\left(I_{B} / I_{B}^{2}, B\right) \text {. }
$$

Proof. a) Clearly ${ }_{0} \operatorname{Hom}_{R}\left(I_{B}, I_{A / B}\right)=0$ and $\left(I_{B}\right)_{v}=\left(I_{A}\right)_{v}$ for all $v \leq m(B)$ by assumption. Since $\left(I_{B^{\prime}}\right)_{v} \subset\left(I_{A}\right)_{v}$, we get $\left(I_{B}\right)_{v}=\left(I_{B^{\prime}}\right)_{v}$ for $v \leq m(B)$ because $H_{B}=H_{B^{\prime}}$. Hence $I_{B^{\prime}}$ contains all minimal generators of $I_{B}$, i.e. $I_{B} \subset I_{B^{\prime}}$, and we get equality by using $H_{B}=H_{B^{\prime}}$.

b) By Proposition 4(i) there is an open subset $U$ of $\operatorname{GradAlg}\left(H_{B}, H_{A}\right)$ such that the restriction of $q$ to $U$ is smooth. By possibly shrinking $U$, we get $m\left(B^{\prime}\right) \leq m(B)$ for any $k$-point $\left(B^{\prime}\right) \in q(U)$ by semicontinuity. Hence for any $\left(B^{\prime} \rightarrow A^{\prime}\right) \in U$ we get $\left(I_{A^{\prime} / B^{\prime}}\right)_{v}=0$ for all $v \leq m\left(B^{\prime}\right)$ because $H_{B}=H_{B^{\prime}}$ and $H_{A}=H_{A^{\prime}}$, and we see by (i) that $p$ is unramified on $U$ and injective on the $k$-points of $p^{-1}(U)$. Now we do not need to use the theorem of generic smoothness because the codimension of the stratum must be $\operatorname{dim}_{(A)} \operatorname{GradAlg}^{H_{A}}-\operatorname{dim}_{(B \rightarrow A)} \operatorname{GradAlg}\left(H_{A}, H_{B}\right)$, and we conclude by (10) and (12) and the unobstructedness of $A$ and $B \rightarrow A$.

In the next section we will need to combine (91) with the following result, which in fact is a corollary (29, Cor. 9.10) of a geometric variation (29, Thm. 9.4) of Theorem $5 \mathrm{~B})$. Below $s(C / F)$ is the minimal degree of the generators of the homogeneous ideal $I_{C / F}$.

Proposition 8. Let $C_{0} \subset \mathbb{P}^{c+1}$ be an arithmetically Cohen-Macaulay curve of degree $d_{0}$ and arithmetic genus $g_{0}$, sitting on a smooth rational surface $F \subset \mathbb{P}^{c+1}$ of degree $f$. Let $K$ be the canonical divisor of $F$. If $t$ is an integer such that $t \geq 5-s\left(C_{0} / F\right)$ and $t>\left(C_{0} \cdot K\right) / d_{0}$, then any member $C=\operatorname{Proj}(B)$ of the linear system $\left|C_{0}+t H\right|$ is unobstructed and

$$
\operatorname{dim}_{(C)} \operatorname{Hilb}^{p}\left(\mathbb{P}^{c+1}\right)=h^{0}\left(\mathcal{N}_{F}\right)+g_{0}-C_{0} \cdot K+t\left(d_{0}+c+1-f / 2\right)+t^{2} f / 2-1 .
$$

Moreover, if $F$ is nondegenerate, then $h^{0}\left(\mathcal{N}_{F}\right)=c^{2}+4 c+13-2 K^{2}$.

We finish this section by looking more closely at deformations of an Artinian algebra $A$. First we recall the representability of the functor (sheaf) $\operatorname{GRADALG}^{H}(-)$, locally given by letting $\operatorname{GRADALG}^{H}(\operatorname{Spec}(S))(S$ a noetherian $k$-algebra) be the 
set of graded $S$-flat quotients $R_{S} \rightarrow A_{S}$ such that $H_{A_{s}}=H$ and $\operatorname{depth}_{\mathfrak{m}} A_{s} \geq$ $\min \left(1, \operatorname{dim} A_{s}\right)$ for any $s \in \operatorname{Spec}(S)$. Here $R_{S}=R \otimes_{k} S$ and $A_{s}$ is the "fiber" of $\operatorname{Spec}\left(A_{S}\right) \rightarrow \operatorname{Spec}(S)$ at $s \in \operatorname{Spec}(S)$. To simplify and clarify 27] (being more careful with the topologies involved), we sketch proofs. Note that the representability below (27], Thm. 1.1) has later been generalized by Haiman and Sturmfels ([19]).

Proposition 9. The functor $\mathrm{GRADALG}^{H}(-)$ is representable. Its representing object, $\operatorname{GradAlg}^{H}(R)$, is a $k$-scheme of finite type.

Proof. Here we sketch a proof which is slightly different from that in [27] in the Artinian case (i.e. when $H(v)=0$ for $v>>0$ ). Indeed the flattening stratification described in 37] and Grothendieck's representability theorems of the Hilbert functor are the results we need to conclude. In the non-Artinian case, $H(v)$ is a polynomial $p(v)$ for $v>>0$, and $\operatorname{GradAlg}^{H}(R)$ is the stratum of $\operatorname{Hilb}^{p}\left(\mathbb{P}^{n+c-1}\right)$ of constant postulation (cf. [27, Thm. 1.1, for details). If $H(v)=0$ for $v>>0$ and $t=$ $\sum_{v} H(v)$, then there exists a scheme Hilb $:=\operatorname{Hilb}^{t}\left(\mathbb{A}^{n+c}\right)$ of finite type which parametrizes quotients $R \rightarrow A$ of length $t$. The restriction (pullback) $R_{S} \rightarrow A_{S}$ of the universal quotient to $\operatorname{Spec}(S) \rightarrow$ Hilb defines the subscheme $\operatorname{GradAlg}^{H}(R)$ of Hilb by requiring $\operatorname{ker}\left(\left(R_{S}\right)_{v} \rightarrow A_{S}\right)$ to be flat over $S$, of $\operatorname{rank} \operatorname{dim} R_{v}-H(v)$ for every $0 \leq v \leq t$ at each fiber of $\operatorname{Spec}(S)$. Since such a subscheme $\operatorname{GradAlg}^{H}(R)$ of finite type exists by [37, Lect. 8, or [33], VI, Prop. 1.1, we are done.

Remark 10. We may in the case $H(v)=0$ for $v>>0$ use flattening stratification to show the existence of the scheme $\mathrm{Z}(\mathrm{H})$ of not necessarily graded Artinian quotients $A$ of $R$ with Hilbert function $H$ (by requiring $\operatorname{gr}\left(A_{S}\right)$ to be $S$-flat, of Hilbert function $H$ at each fiber of $\operatorname{Spec}(S)$ ), to get $\mathrm{Z}(\mathrm{H})$ as a locally closed subscheme (stratum) of $\operatorname{Hilb}^{t}\left(\mathbb{A}^{n+c}\right)$. Its local deformation functor at a quotient $A \simeq R / I_{A}$ (with $\sqrt{I_{A}}=\mathfrak{m}$ ) of $R$, defined on the category of local Artinian $k$-algebras with residue field $k$, consists of quotients $A_{S}$ of $R_{S}=S\left[X_{1}, \ldots, X_{n+c}\right]$ (or equivalently, of $\left.S\left[\left[X_{1}, \ldots, X_{n+c}\right]\right]\right)$ such that $A_{S}$ and $\operatorname{gr}\left(A_{S}\right)$ are deformations of $A$ and $\operatorname{gr}(A)$, respectively. If $R \rightarrow A$ is graded and satisfies ${ }_{v} \mathrm{H}^{2}(R, A, A)=0$ for $v \geq 0$, then [27, Thm. 1.10 and Rem. 1.11, shows that $\mathrm{Z}(\mathrm{H})$ is smooth at $(R \rightarrow A)$ and $\operatorname{dim}_{(A)} \mathrm{Z}(\mathrm{H})=\sum_{v \geq 0} \operatorname{hom}_{R}\left(I_{A}, A\right)$.

Inside $\operatorname{GradAlg}^{H}(R)$ there is an open set (and hence a scheme $\operatorname{Gor}_{c}^{H}(R)$ with induced scheme structure) consisting of graded Gorenstein quotients $R \rightarrow A$ with Hilbert function $H . \operatorname{Gor}_{c}^{H}(R)$ is empty unless the difference $\Delta^{\operatorname{dim} A} H$, i.e. the $h$-vector of $A$, is a symmetric Gorenstein sequence. In the Artinian case there is another known scheme parametrizing graded Gorenstein quotients with suitable Hilbert function $H$, namely the determinantal loci PGor $(H)$ parametrizing forms $F$ of degree $s$ in $\mathbb{P}^{N}, N=\left(\begin{array}{c}s+n+c-1 \\ n+c-1\end{array}\right)-1$, given by requiring the "catalecticant matrices" of $F$ to have ranks given by the Hilbert function (see Iarrobino-Kanev's book [21, Sect. 1.1). We denote the restriction of the generic $s$-form of $\mathbb{P}^{N}$ to PGor $(H)$ by $F_{\mathrm{PGor}(H)}$ (21], Def. 1.3). Then the underlying sets of closed points of $\operatorname{Gor}_{c}^{H}(R)$ and PGor $(H)$ are the same by apolarity (the Macaulay correspondence), and one may see from the proof of our next result that they are in fact isomorphic as topological spaces. Moreover, since Iarrobino and Kanev have proved that the tangent space of $\mathrm{PGor}(H)$ at $A$ is isomorphic to ${ }_{0} \operatorname{Hom}_{A}\left(I_{A} / I_{A}^{2}, A\right)$ (21], Thm. 3.9) and it is well known that the tangent space of $\operatorname{GradAlg}^{H}(R)$ is the same group [24, we get the following theorem. 
Theorem 11. Let $R \rightarrow A$ be a graded Artinian Gorenstein quotient with Hilbert function $H$. Then $\operatorname{dim}_{(A)} \operatorname{GradAlg}^{H}(R)=\operatorname{dim}_{(A)} \operatorname{PGor}(H)$. Hence PGor $(H)$ is smooth at $(R \rightarrow A)$ if and only if $\operatorname{GradAlg}^{H}(R)$ is smooth at $(R \rightarrow A)$. In particular $\operatorname{PGor}(H)$ is smooth at $(R \rightarrow A)$ provided ${ }_{0} \mathrm{H}^{2}(R, A, A)=0$, i.e. provided the natural surjection $\left(S_{2} I_{A}\right)_{s} \rightarrow\left(I_{A}^{2}\right)_{s}$ from the second symmetric power to the second power of $I_{A}$ is an isomorphism in the socle degree $s$ of $A$.

Proof. By duality, ${ }_{0} \mathrm{H}^{2}(R, A, A)=0$ is equivalent to ${ }_{s} \mathrm{H}_{2}(R, A, A)=0$ which again is equivalent to $\left(S_{2} I_{A}\right)_{s} \simeq\left(I_{A}^{2}\right)_{s}$ by (28) below. Hence we only need to prove $\operatorname{dim}_{(A)} \operatorname{GradAlg}^{H}(R)=\operatorname{dim}_{(A)} \operatorname{PGor}(H)$, because we know their tangent spaces are isomorphic. Let $V \subset$ PGor $(H)$ be a closed irreducible subset (e.g. of the form $V=\operatorname{Spec}(T)$ ), and let $V$ have the reduced scheme structure. By the definition of PGor $(H)$, the restriction $F_{V}$ of the "universal" $s$-form $F_{\mathrm{PGor}(H)}$ to $V$ defines via apolarity a family of graded Artinian Gorenstein quotients over $V$ (e.g. the family $A_{T} \simeq R_{T} \circ F_{V}$ over $V=\operatorname{Spec}(T)$, where $R_{T}$ is interpreted as the $T$-algebra of contractions, "partial derivations without coefficients") with constant Hilbert function $H$. Since $V$ is integral, it follows that the family (e.g. the morphism $\left.\operatorname{Spec}\left(A_{T}\right) \rightarrow \operatorname{Spec}(T)\right)$ is flat (37, Lect. 6). Hence we have a morphism $\pi: V \rightarrow$ $\operatorname{GradAlg}^{H}(R)$ by the universal property of $\operatorname{GradAlg}^{H}(R) . \pi(V)$ is irreducible and closed in $\operatorname{Gor}_{c}^{H}(R)$ (it is closed because an "inverse" $\left(\operatorname{Gor}_{c}^{H}(R)\right)_{r e d} \rightarrow \operatorname{PGor}(H)$ on closed points exists by [21], Ch. 8). So chains of closed irreducible subsets in $\operatorname{PGor}(H)$ and $\operatorname{Gor}_{c}^{H}(R)$ correspond, and we are done.

Problem 12. It would be interesting to prove that PGor $(H)$ and $\operatorname{Gor}_{c}^{H}(R)$ are isomorphic as schemes; cf. [27, Rem.1.9. The arguments in the proof above, which easily lead to an isomorphism of $\operatorname{PGor}(H)$ and $\operatorname{Gor}_{c}^{H}(R)$ as topological spaces, are mainly contained in [27, Rem. 1.9. In that remark we also claimed that these arguments showed the existence of a natural morphism $\Pi: \operatorname{PGor}(H) \rightarrow \operatorname{Gor}_{c}^{H}(R)$. Unfortunately an index "red" is missing in Rem.1.9, i.e. from the arguments we can only be sure of the existence of a natural morphism $\pi:(\operatorname{PGor}(H))_{r e d} \rightarrow \operatorname{Gor}_{c}^{H}(R)$, and the existence of $\Pi$ above remains to be proved.

\section{FAMILIES OF CODIMENSION ONE GORENSTEIN QUOTIENTS of Cohen-Macaulay Algebras}

The main purpose of this paper is to study families of graded Gorenstein quotients $A$ obtained by taking a regular section $\sigma$ of the anticanonical system $\left(K_{B}^{*}\right)_{s}$, i.e. quotients $A$ given by

$$
0 \rightarrow K_{B}(-s) \stackrel{\sigma^{*}}{\rightarrow} B \rightarrow A \rightarrow 0
$$

for some integer $s$. Our main results apply to Artinian Gorenstein algebras as well as to Gorenstein quotients of higher dimension. In what follows $B$ is CohenMacaulay. In order to make the description of $A$ in (13) meaningful, we must suppose that $B=R / I_{B}$ is generically Gorenstein (cf. [29], Lemma 5.2 and 5.4). Then we say " $A$ is a codimension one quotient of $B$, well-defined by a twist of the canonical module". If $A$ is such a quotient of $B$, then a stratum of quotients given by (13) around $(A)$ is just a maximal (flat) family consisting of quotients $\left(A^{\prime}\right)$ of GradAlg ${ }^{H_{A}}(R)$ for which there exists a factorization $B^{\prime} \rightarrow A^{\prime}$ as in (13), where $\left(B^{\prime}\right)$ (resp. the sections) belong to some open neighbourhood of $(B)$ in $\operatorname{GradAlg}^{H_{B}}(R)$ 
(resp. of $\sigma$ in the linear system $\left.\left(K_{B^{\prime}}^{*}\right)_{s} \subset \mathrm{H}^{0}\left(\operatorname{Proj}\left(B^{\prime}\right), \widetilde{K_{B^{\prime}}}{ }^{*}(s)\right)\right)$, making each $A^{\prime}$ well-defined in the sense above. Looking to the proof of Proposition 13, one may see that such a stratum is just the image $p(U)$ of some open $U$ of $\operatorname{Grad} \operatorname{Alg}\left(H_{B}, H_{A}\right)$ containing $(B \rightarrow A)$ via the second projection $p$, provided ${ }_{s} \operatorname{Ext}_{B}^{1}\left(S_{2}\left(K_{B}\right), K_{B}\right)=0$.

The following result determines $\operatorname{dim}_{(A)} \operatorname{GradAlg}^{H_{A}}(R)$ in terms of invariants of $B$, such as $N_{B}:=\operatorname{Hom}_{B}\left(I_{B} / I_{B}^{2}, B\right)$ and

$$
K_{B}^{*}:=\operatorname{Hom}_{B}\left(K_{B}, B\right) \simeq \operatorname{Hom}_{B}\left(S_{2}\left(K_{B}\right), K_{B}\right)
$$

(here $S_{2}\left(K_{B}\right)$ is the second symmetric power of $\left.K_{B}\right)$, and leads to the main theorems of this section. Since Proposition 13 is a variation of Theorem 5 with $I_{A / B}=K_{B}(-s)$, Remark 6 (except the first sentence of Remark 6b) ) will apply as a remark to Proposition 13 as well.

Proposition 13. Let $B$ be a generically Gorenstein, graded Cohen-Macaulay quotient of a polynomial ring $R$, let $A$ be a codimension one quotient of $B$, defined by (13) for some $s$, and let ${ }_{s} \operatorname{Ext}_{B}^{1}\left(S_{2}\left(K_{B}\right), K_{B}\right)=0$.

A) If ${ }_{0} \operatorname{Ext}_{B}^{1}\left(I_{B} / I_{B}^{2}, A\right)=0$ and $\left(I_{B}\right)_{\wp}$ is syzygetic for any graded prime $\wp$ of Ass $(A)$, then $A$ is unobstructed as a graded $R$-algebra, $A$ is Gorenstein and

$$
\operatorname{dim}_{(A)} \operatorname{GradAlg}^{H_{A}}(R)=\operatorname{dim}\left(N_{B}\right)_{0}+\operatorname{dim}\left(K_{B}^{*}\right)_{s}-1-\delta(B)_{-s}-{ }_{0} \operatorname{ext}_{B}^{1}\left(I_{B} / I_{B}^{2}, B\right),
$$

where $\delta(B)_{-s}={ }_{-s} \operatorname{hom}_{R}\left(I_{B}, K_{B}\right)-{ }_{-s} \operatorname{ext}_{B}^{1}\left(I_{B} / I_{B}^{2}, K_{B}\right)$. Moreover if $B$ is unobstructed as a graded $R$-algebra and char $(k)=0$, then the codimension of the $H_{B}$-stratum of $A$ at $(B \rightarrow A)$ is

$$
{ }_{-s} \operatorname{ext}_{B}^{1}\left(I_{B} / I_{B}^{2}, K_{B}\right)-{ }_{0} \operatorname{ext}_{B}^{1}\left(I_{B} / I_{B}^{2}, B\right) .
$$

This number also equals the codimension of the stratum of quotients given by (13) at $(A)$.

B) If ${ }_{-s} \operatorname{Ext}_{B}^{1}\left(I_{B} / I_{B}^{2}, K_{B}\right)=0$ and $I_{B}$ is generically syzygetic, then $A$ is Gorenstein and $H_{B}$-generic. Moreover $A$ is unobstructed as a graded $R$-algebra if and only if $B$ is unobstructed as a graded $R$-algebra. Indeed

${ }_{0} \operatorname{hom}_{R}\left(I_{A}, A\right)-\operatorname{dim}_{(A)} \operatorname{GradAlg}^{H_{A}}(R)={ }_{0} \operatorname{hom}_{R}\left(I_{B}, B\right)-\operatorname{dim}_{(B)} \operatorname{GradAlg}^{H_{B}}(R)$, and

$\operatorname{dim}_{(A)} \operatorname{GradAlg}^{H_{A}}(R)=\operatorname{dim}_{(B)} \operatorname{GradAlg}^{H_{B}}(R)+\operatorname{dim}\left(K_{B}^{*}\right)_{s}-1-{ }_{-s} \operatorname{hom}_{R}\left(I_{B}, K_{B}\right)$.

Remark 14. a) With the notations $0 \rightarrow \bigoplus_{i} R\left(-n_{c, i}\right) \rightarrow \ldots \rightarrow \bigoplus_{i} R\left(-n_{1, i}\right) \rightarrow I_{B} \rightarrow$ 0 of (6) , recalling $\operatorname{dim} R=n+c$ and $\operatorname{dim} B=n$, we claim that

$$
\delta(B)_{-s}=0 \text { provided } s>2 \operatorname{reg}\left(I_{B}\right)-n .
$$

By (7) it suffices to prove ${ }_{-s} \operatorname{Ext}_{B}^{i}\left(I_{B} / I_{B}^{2}, K_{B}\right)=0$ for $i=0$ and 1 provided $s>\max n_{c, i}+\max n_{2, i}-n-c$. Indeed dualizing (6) we get a surjection $\bigoplus_{i} R\left(n_{c, i}\right) \rightarrow$ $K_{B}(n+c)$, and it follows that $\left(K_{B}\right)_{-v}=0$ for $v>\max n_{c, i}-n-c$. We conclude by applying ${ }_{0} \operatorname{Hom}_{B}\left(-, K_{B}\right)$ to the right-exact sequence $\bigoplus_{i} B\left(-n_{2, i}\right) \rightarrow$ $\bigoplus_{i} B\left(-n_{1, i}\right) \rightarrow I_{B} / I_{B}^{2} \rightarrow 0$ and the definition of $\operatorname{Ext}_{B}^{i}\left(I_{B} / I_{B}^{2}, K_{B}\right)$.

b) If $\operatorname{dim} B=1$, we have $\operatorname{Ext}_{B}^{i}\left(-, K_{B}\right)=0$ for $i \geq 2$ by Gorenstein duality. In this case ${ }_{0} \operatorname{Ext}_{B}^{1}\left(I_{B} / I_{B}^{2}, B\right)=0$ implies ${ }_{0} \operatorname{Ext}_{B}^{1}\left(I_{B} / I_{B}^{2}, A\right)=0$ by Remark 6 r). Moreover looking to Remark [6]) and using that $A \simeq K_{A}(-s)$ and Gorenstein duality, we may weaken " $I_{B}$ is syzygetic for any graded prime $\wp$ of $A s s(A)$ " to " $\left(\mathrm{H}_{2}(R, B, B) \otimes_{B} A\right)_{s}=0 "$. Hence if we suppose

$$
{ }_{0} \operatorname{Ext}_{B}^{1}\left(I_{B} / I_{B}^{2}, B\right)=\left(\mathrm{H}_{2}(R, B, B) \otimes_{B} A\right)_{s}=0
$$


we still have the conclusion concerning the unobstructedness of $A$ and the formula for $\operatorname{dim}_{(A)} \operatorname{GradAlg}^{H_{A}}(R)$ of Proposition [13A). Furthermore if $I_{B}$ is generically syzygetic (this is always true if $c \leq 3$ since $B$ is generically Gorenstein; cf. the proof of Theorem [16), then $B$ is unobstructed as a graded algebra and the codimension formula of Proposition [13A) holds as well (in the characteristic zero case).

c) Slightly reformulating Proposition [13A) (by replacing the vanishing of the two ${ }_{0} \operatorname{Ext}_{B}^{1}$ groups by the corresponding vanishing of $\operatorname{Ext}_{B}^{1}$ ) one may use the proof below to show that $A$ is unobstructed as an $R$-algebra in the sense $\mathrm{H}^{2}(R, A, A)=0$; cf. Remark 6. A further slight reformulation (replacing Proj by Spec and skipping the twists) allows us to prove a corresponding result for non-graded objects as well. If $B$ is licci, then the three $\operatorname{Ext}_{B}$ groups, $\operatorname{Ext}_{B}^{1}\left(S_{2}\left(K_{B}\right), K_{B}\right), \operatorname{Ext}_{B}^{1}\left(I_{B} / I_{B}^{2}, B\right)$ and $\operatorname{Ext}_{B}^{2}\left(I_{B} / I_{B}^{2}, K_{B}\right)$, vanish ([5], 20], 38, Thm. 4.2.6, or Corollary 37 of this paper), and we get the unobstructedness of the Gorenstein quotient $A$ by Remark 6r). Indeed $S_{2}\left(K_{B}\right)$ is Cohen-Macaulay if $B$ is licci. In the interesting codimension $c=3$ case, the Cohen-Macaulayness of $S_{2}\left(K_{B}\right)$ and of the Koszul homology group $\mathrm{H}_{1}=\mathrm{H}_{1}\left(I_{B}\right)$ are equivalent 38], Thm. 4.2.9. This Cohen-Macaulayness holds in particular if the Cohen-Macaulay type of $B$ is 2 [38], Cor. 4.2.11.

d) If $I_{B}$ is generically syzygetic, then $\operatorname{Hom}_{B}\left(\mathrm{H}_{2}(R, B, B), K_{B}\right)=0$. Hence applying $\operatorname{Hom}_{B}\left(-, K_{B}\right)$ to (4), we see that ${ }_{-s} \operatorname{Ext}_{B}^{2}\left(I_{B} / I_{B}^{2}, K_{B}\right) \hookrightarrow_{-s} \operatorname{Ext}_{B}^{1}\left(\mathrm{H}_{1}, K_{B}\right)$ is injective and that

$$
\delta(B)_{-s}=\operatorname{dim}\left(G_{1}^{*} \otimes_{R} K_{B}\right)_{-s}-{ }_{-s} \operatorname{hom}_{B}\left(\mathrm{H}_{1}, K_{B}\right),
$$

allowing us to restate the dimension formula in terms of $\mathrm{H}_{1}$ and $G_{1}$. If $B$ is licci, then ${ }_{-s} \operatorname{Hom}_{B}\left(\mathrm{H}_{1}, K_{B}\right) \simeq\left(\mathrm{H}_{r-1}\right)_{-t-s}$ by [30, Prop. 18, where $r$ and $t$ are given by $\left.\bigwedge^{r}{\widetilde{\mathrm{H}_{1}}}_{\left.\right|_{U}} \simeq \widetilde{K}_{B}(t)\right|_{U}$.

Proof. Note that we can show that $A \simeq K_{A}(-s)$ and hence that $A$ is Gorenstein by applying the mapping cone construction to (13) (or see [29], Lemma 5.2 and 5.4). We claim that Proposition 13 above follows from Theorem 5 and Remark 6 by letting $I_{A / B}=K_{B}(-s)$ and by paying some extra attention to the codimension of the strata. Indeed it is straightforward to obtain the assumptions of Theorem 5 from the assumptions of Proposition 13, except possibly the assumption ${ }_{0} \operatorname{Ext}_{B}{ }_{B}\left(I_{A / B}, A\right)=0$ of Theorem 5. Suppose temporarily that $B$ is Gorenstein in codimension $\leq 1$. Then ${ }_{0} \operatorname{Ext}_{B}^{1}\left(K_{B}(-s), B\right) \simeq{ }_{0} \operatorname{Ext}_{B}^{1}\left(K_{B} \otimes K_{B}(-s), K_{B}\right) \simeq{ }_{0} \operatorname{Ext}_{B}^{1}\left(S_{2}\left(K_{B}\right)(-s), K_{B}\right)=0$ by a well-known spectral sequence ([18, Satz 1.2). Moreover, since $K_{B}$ is a maximal Cohen-Macaulay $B$-module, one knows ${ }_{0} \operatorname{Ext}_{B}^{i}\left(K_{B}, K_{B}\right)=0$ for $i>0$ and we get ${ }_{0} \operatorname{Ext}_{B}^{1}\left(K_{B}(-s), A\right)=0$ by applying ${ }_{0} \operatorname{Hom}\left(K_{B}(-s),-\right)$ to (13), i.e. we get the claim. In the general case where $B$ is generically Gorenstein we get the conclusion of Theorem 5 by combining Lemma 15 and Remark [6]), recalling $K_{B}(-s) \simeq I_{A / B}$. Furthermore to get the dimension formulas we note that ${ }_{0} \operatorname{Hom}\left(K_{B}(-s), K_{B}(-s)\right)$ is one dimensional. Hence by again applying ${ }_{0} \operatorname{Hom}\left(K_{B}(-s),-\right)$ to (13), we get the dimension formulas from the corresponding formulas of Theorem 5 .

It remains to see that the codimension may also be computed from the stratum of quotients given by (13). Now looking to the definition of the codimension of the $H_{B^{-}}$ stratum of $A$ at $(B \rightarrow A)$ before Theorem $\left[5\right.$ it suffices to show that any $\left(B^{\prime} \rightarrow A^{\prime}\right)$ in a small enough open neighbourhood of $t:=(B \rightarrow A)$ in $G:=\operatorname{GradAlg}\left(H_{B}, H_{A}\right)$ is given by (13). Let $\left(S, m_{S}\right)$ be the local ring of $G$ at $t$ and let $S_{i}=S / m_{S}^{i}$. Since $G$ is a scheme of finite type which represents a certain functor of graded flat quotients, 
there exists a universal quotient whose pullback to $\operatorname{Spec}(S)$ is denoted by $B_{S} \rightarrow A_{S}$. Its kernel $I_{A_{S} / B_{S}}$ is a flat deformation of the $B$-module $I_{A / B} \simeq K_{B}(-s)$ to $S$. By the theory of deformations of the module $I_{A / B}$ its tangent and obstruction spaces are given by ${ }_{0} \operatorname{Ext}_{B}^{i}\left(K_{B}(-s), K_{B}(-s)\right)$ for $i=1$ and 2, respectively. Since both of these Ext-groups vanish, there is a unique deformation of $I_{A / B}$ to $B_{S_{i}}$, i.e. we get isomorphisms $l_{i}: I_{A_{S} / B_{S}} \otimes_{S} S_{i} \simeq K_{B_{S}}(-s) \otimes_{S} S_{i}$ which commute with $l_{i-1}$ because $K_{B_{S}}:=\operatorname{Ext}_{R_{S}}^{c}\left(B_{S}, R_{S}(-n-c)\right)$, where $R_{S}:=R \otimes_{k} S$, is $S$-flat (cf. [23], Prop. A1). It suffices to show that there exists a morphism $l: I_{A_{S} / B_{S}} \rightarrow K_{B_{S}}(-s)$ which reduces to $l_{i} \bmod m_{S}^{i}$ because $l$ must then be an isomorphism which extends to an isomorphism in an open neighbourhood of $t$ in $G$. To show the existence of $l$, we claim that $K_{B_{S}}^{*}(s) \rightarrow K_{B}^{*}(s)$ is surjective. By the proof of [26], Prop. 2.4, it suffices to show that $\eta_{i}: K_{B_{S_{i}}}^{*}(s) \rightarrow K_{B_{S_{i-1}}}^{*}(s)$ is surjective. If ${ }_{0} \operatorname{Ext}_{B}^{1}\left(K_{B}(-s), B\right)=0$ (e.g. $B$ is Gorenstein in codimension $\leq 1$; cf. the first part of the proof), this follows by applying ${ }_{0} \operatorname{Hom}\left(K_{B_{S_{i}}}(-s),-\right)$ onto $0 \rightarrow B \otimes_{k} \mathfrak{a} \rightarrow B_{S_{i}} \rightarrow B_{S_{i-1}} \rightarrow 0$ where $\mathfrak{a}:=m_{S}^{i-1} / m_{S}^{i}$. In the general case, replacing every $B$ by $A$ in the latter sequence and applying ${ }_{0} \operatorname{Hom}\left(K_{B_{S_{i}}}(-s),-\right) \simeq{ }_{0} \operatorname{Hom}\left(I_{A_{S_{i}} / B_{S_{i}}} \otimes_{B_{S_{i}}} A_{S_{i}},-\right)$ to that sequence, we get the surjectivity of $\eta_{i}$ from ${ }_{0} \operatorname{Ext}_{A}^{1}\left(I_{A / B} / I_{A / B}^{2}, A\right)=0$ and ${ }_{0} \operatorname{Ext}_{B}^{1}\left(K_{B}(-s), K_{B}(-s)\right)=0$. Note that we have the last-mentioned vanishings by (5), Lemma 15, and ${ }_{s} \operatorname{Ext}^{1}\left(S_{2}\left(K_{B}\right), K_{B}\right)=0$, and the claim is proved. Now using the proven claim we get a morphism $K_{B_{S}}(-s) \rightarrow B_{S}$ whose cokernel is an S-flat deformation of $A$. By the universal property of $G$, there is a morphism $\operatorname{Spec}(S) \rightarrow \operatorname{Spec}(S)$ whose pullback induces the desired map $I_{A_{S} / B_{S}} \rightarrow K_{B_{S}}(-s)$, and the proof is complete.

Lemma 15. Let $B$ be a graded Cohen-Macaulay quotient of $R$ and let $A \simeq B / I_{A / B}$ be a graded Gorenstein quotient such that $A \simeq K_{A}(-s)$ and $\operatorname{dim} B-\operatorname{dim} A=r$. If $r>0$, then ${ }_{0} \mathrm{H}^{2}(B, A, A)$ and ${ }_{-s} \operatorname{Ext}_{B}^{r}\left(S_{2}\left(I_{A / B}\right), K_{B}\right)$ vanish simultaneously.

Proof. Using (5) we see that the vanishing of ${ }_{0} \mathrm{H}^{2}(B, A, A)$ is equivalent to

$$
{ }_{0} \operatorname{Ext}_{A}^{1}\left(I_{A / B} / I_{A / B}^{2}, A\right)=0
$$

and the injectivity of the natural map

$$
{ }_{0} \operatorname{Hom}_{A}\left(\mathrm{H}_{2}(B, A, A), A\right) \hookrightarrow{ }_{0} \operatorname{Ext}_{A}^{2}\left(I_{A / B} / I_{A / B}^{2}, A\right) .
$$

Moreover, since $A \simeq K_{A}(-s)$ we have by Gorenstein duality (applied to both $A$ and $B$ ) that

$$
{ }_{0} \operatorname{Ext}_{A}^{i}\left(I_{A / B} / I_{A / B}^{2}, A\right) \simeq{ }_{s} \mathrm{H}_{\mathfrak{m}}^{\operatorname{dim} A-i}\left(I_{A / B} / I_{A / B}^{2}\right)^{\vee} \simeq{ }_{-s} \operatorname{Ext}_{B}^{i+r-1}\left(I_{A / B}^{2}, K_{B}\right)
$$

for $i \geq 1$ because the middle term is isomorphic to ${ }_{s} \mathrm{H}_{\mathfrak{m}}^{\operatorname{dim} A+1-i}\left(I_{A / B}^{2}\right)$ by the long exact sequence of local cohomology applied to $0 \rightarrow I_{A / B}^{2} \rightarrow I_{A / B} \rightarrow I_{A / B} / I_{A / B}^{2} \rightarrow$ 0 . Correspondingly, ${ }_{0} \operatorname{Hom}_{A}\left(\mathrm{H}_{2}(B, A, A), A\right) \simeq{ }_{-s} \operatorname{Ext}_{B}^{r}\left(\mathrm{H}_{2}(B, A, A), K_{B}\right)$. Hence ${ }_{0} \mathrm{H}^{2}(B, A, A)=0$ is equivalent to ${ }_{-} \operatorname{Ext}_{B}^{r}\left(I_{A / B}^{2}, K_{B}\right)=0$ and the injectivity of the natural map ${ }_{-s} \operatorname{Ext}_{B}^{r}\left(\mathrm{H}_{2}(B, A, A), K_{B}\right) \hookrightarrow{ }_{-s} \operatorname{Ext}_{B}^{r+1}\left(I_{A / B}^{2}, K_{B}\right)$. Recalling the exact sequence

$$
0 \rightarrow \mathrm{H}_{2}(B, A, A) \rightarrow S_{2}\left(I_{A / B}\right) \rightarrow I_{A / B}^{2} \rightarrow 0
$$

(cf. [1, p. 106, or 38, Sect. 2.1) and that ${ }_{-s} \operatorname{Ext}_{B}^{r-1}\left(\mathrm{H}_{2}(B, A, A), K_{B}\right)=0$ since $\mathrm{H}_{2}(B, A, A)$ is supported at the codimension $r$ quotient $A$ of $B$, we get the 
conclusion of Lemma 15 by the long exact sequence of ${ }_{-s} \operatorname{Ext}_{B}^{i}\left(-, K_{B}\right)$ applied to (14).

Theorem 16. Let $B=R / I_{B}$ be a graded Cohen-Macaulay quotient of a polynomial ring $R$, and let $A$ be a graded codimension one quotient of $B$, well-defined by a twist of the canonical module $K_{B}$.

A) If $B$ is licci, then $A$ is unobstructed as a graded $R$-algebra, $A$ is Gorenstein and,

$$
\operatorname{dim}_{(A)} \operatorname{GradAlg}^{H_{A}}(R)=\operatorname{dim}_{(B)} \operatorname{GradAlg}^{H_{B}}(R)+\operatorname{dim}\left(K_{B}^{*}\right)_{s}-1-\delta(B)_{-s},
$$

where

$$
\delta(B)_{-s}={ }_{-s} \operatorname{hom}_{B}\left(I_{B} / I_{B}^{2}, K_{B}\right)-{ }_{-s} \operatorname{ext}_{B}^{1}\left(I_{B} / I_{B}^{2}, K_{B}\right) .
$$

Moreover, if $\operatorname{char}(k)=0$, then the codimension of the $H_{B}$-stratum of $A$ at $(B \rightarrow A)$ is ${ }_{-s} \operatorname{ext}_{B}^{1}\left(I_{B} / I_{B}^{2}, K_{B}\right)$. This number also equals the codimension of the stratum of quotients given by (13) around $(A)$.

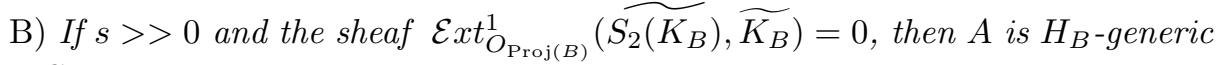
and Gorenstein, and

$$
\operatorname{dim}_{(A)} \operatorname{GradAlg}^{H_{A}}(R)=\operatorname{dim}_{(B)} \operatorname{GradAlg}^{H_{B}}(R)+\operatorname{dim}\left(K_{B}^{*}\right)_{s}-1 .
$$

Moreover, $A$ is unobstructed as a graded $R$-algebra if and only if $B$ is unobstructed as a graded $R$-algebra.

Note that if $Y=\operatorname{Proj}(B)$ is locally licci, then the sheaf $\widetilde{S_{2}\left(K_{B}\right)}$ is maximally Cohen-Macaulay by Remark [14 $)$, i.e. $\mathcal{E} x t_{O_{Y}}^{1}\left(\widetilde{S_{2}\left(K_{B}\right)}, \widetilde{K_{B}}\right)=0$, and we get Theorem 1 The sheaf also vanishes if $Y$ is (locally) Gorenstein, e.g. if $\operatorname{dim} Y=0$.

Proof. A) Since $B$ is licci and generically Gorenstein, $I_{B}$ is generically syzygetic by [29], Prop. 6.17, which implies $\mathrm{H}^{2}(R, B, B)_{\wp}=0$ provided $\operatorname{dim} B_{\wp}=0$. This in turn is equivalent to $\mathrm{H}_{2}(R, B, B)_{\wp}=0$ by Gorenstein duality and the spectral sequence relating algebra-homology to algebra-cohomology, i.e. $I_{B}$ is generically syzygetic. Then $I_{B}$ is in fact syzygetic by (4) because $\mathrm{H}_{1}$ is a maximal CohenMacaulay module in the licci case. Since all $\operatorname{Ext}_{B}^{1}$ groups of Proposition 13A) vanish by Remark 14k) and Remark 6r), we conclude by Proposition 13 ,

B) If $\mathcal{E} x t_{O_{Y}}^{1}\left(\widetilde{S_{2}\left(K_{B}\right)}, \widetilde{K_{B}}\right)=0$, we see that ${ }_{s} \operatorname{Ext}_{B}^{1}\left(S_{2}\left(K_{B}\right), K_{B}\right)$ vanishes for large $s$ because of the exact sequence

$$
\begin{aligned}
& \rightarrow{ }_{s} \operatorname{Hom}_{B}\left(S_{2}\left(K_{B}\right), \mathrm{H}_{\mathfrak{m}}^{1}\left(K_{B}\right)\right) \rightarrow{ }_{s} \operatorname{Ext}_{B}^{1}\left(S_{2}\left(K_{B}\right), K_{B}\right) \\
& \rightarrow \mathrm{H}^{1}\left(Y, \mathcal{H o m}_{B}\left(\widetilde{S_{2}\left(K_{B}\right)}, \widetilde{K_{B}}\right)(s)\right)
\end{aligned}
$$

(which is analogous to (3) ). Since the vanishing of ${ }_{-s} \operatorname{Hom}_{B}\left(\mathrm{H}_{2}(R, B, B), K_{B}\right)=0$ and ${ }_{-s} \operatorname{Ext}_{B}^{i}\left(I_{B} / I_{B}^{2}, K_{B}\right), i=0,1$, are obviously true for $s>>0$, we conclude by Proposition [13B) and Remark 6b).

To apply Proposition 13 and Theorem 16, we need to compute $\operatorname{dim}\left(K_{B}^{*}\right)_{s}$ and verify its assumptions. In several cases it is easy to compute $\operatorname{dim}\left(K_{B}^{*}\right)_{s}$ at least for large $s$. For instance if $B$ is 2-dimensional and $C=\operatorname{Proj}(B)$ is smooth, then $\widetilde{K}_{B}^{*}$ is just the tangent sheaf (sheaf of derivations), $\theta_{C}$ of $C$, and we can get the information we need from the vanishing of cohomology and the Riemann-Roch theorem. In this case the most difficult assumption to verify is perhaps the unobstructedness of $B$ 
(or ${ }_{0} \operatorname{Ext}_{B}^{1}\left(I_{B} / I_{B}^{2}, B\right)=0$ ), for which we point out a couple of important cases where the unobstructedness is known:

(1) ${ }_{0} \operatorname{Ext}_{B}^{1}\left(I_{B} / I_{B}^{2}, B\right)=0$, provided $C$ is linked (in possibly several steps) to a generically Gorenstein curve $C^{\prime}=\operatorname{Proj}\left(B^{\prime}\right)$ satisfying ${ }_{0} \operatorname{Ext}_{B^{\prime}}^{1}\left(I_{B^{\prime}} / I_{B^{\prime}}^{2}, B^{\prime}\right)$ $=0$ (cf. next section).

(2) $B$ is unobstructed if $C=\operatorname{Proj}(B)$ sits on a smooth rational surface in $\mathbb{P}^{4}$ and the degree of $C$ is $\geq 24$ ([29], Prop. 9.13; the statement also follows from Proposition 8).

By (1) we get ${ }_{0} \operatorname{Ext}_{B}^{1}\left(I_{B} / I_{B}^{2}, B\right)=0$ if $B$ is licci (e.g. if $C^{\prime}$ above is a complete intersection), or if $C^{\prime}$ is a local complete intersection curve and $\mathrm{H}^{1}\left(\mathcal{N}_{C^{\prime}}\right)=0$ (because ${ }_{0} \operatorname{Ext}_{B^{\prime}}^{1}\left(I_{B^{\prime}} / I_{B^{\prime}}^{2}, B^{\prime}\right) \hookrightarrow \mathrm{H}^{1}\left(\mathcal{N}_{C^{\prime}}\right)$ is injective by (3) $)$. Indeed for a local complete intersection curve $C=\operatorname{Proj}(B)$ we have $\left.\mathrm{H}^{1}\left(\mathcal{N}_{C}(v)\right) \simeq \operatorname{Ext}_{\mathcal{O}_{C}}^{1} \widetilde{\left(I_{B} / I_{B}^{2}\right.}, \widetilde{B}(v)\right)$ and by (3) an exact sequence

$$
\begin{aligned}
{ }_{v} \operatorname{Ext}_{B}^{1}\left(I_{B} / I_{B}^{2}, B\right) & \hookrightarrow \mathrm{H}^{1}\left(\mathcal{N}_{C}(v)\right) \rightarrow{ }_{v} \operatorname{Hom}_{B}\left(I_{B} / I_{B}^{2}, \mathrm{H}_{\mathfrak{m}}^{2}(B)\right) \\
& \rightarrow{ }_{v} \operatorname{Ext}_{B}^{2}\left(I_{B} / I_{B}^{2}, B\right) \rightarrow 0 .
\end{aligned}
$$

We illustrate Theorem 16 by taking a relatively simple example, remarking that far more complex examples can be given by the same methods, by increasing $s$.

Example 17. Let $B$ be the homogeneous coordinate ring of a smooth rational arithmetically Cohen-Macaulay curve $C$ of degree $d=4$ in $\mathbb{P}^{4}=\operatorname{Proj}(R)$, with minimal resolution

$$
0 \rightarrow R(-4)^{3} \rightarrow R(-3)^{8} \rightarrow R(-2)^{6} \rightarrow R \rightarrow B \rightarrow 0,
$$

and let $A$ be defined by a regular section of $\left(K_{B}^{*}\right)_{s}$ for $s \geq 0$. Then $C$ is the determinantal curve given by the maximal minors of a 2 by 4 matrix with linear entries, and it is straightforward to see that the Hilbert function $H_{A}$ of $A$ is given by $\operatorname{dim} A_{v}=4 v+1$ for $0 \leq v \leq s$ and $\operatorname{dim} A_{v}=4 s+2$ for $v>s$. Applying $\operatorname{Hom}_{R}(-, R(-5))$ onto (16) we get the minimal resolution of $K_{B}$, which combined with (13) and the mapping cone construction yields the resolution

$$
\begin{aligned}
0 & \rightarrow R(-s-5) \rightarrow R(-s-3)^{6} \oplus R(-4)^{3} \rightarrow R(-s-2)^{8} \oplus R(-3)^{8} \\
& \rightarrow R(-s-1)^{3} \oplus R(-2)^{6} \rightarrow I_{A} \rightarrow 0 .
\end{aligned}
$$

Even though $B$ is not licci, Theorem 16 applies for $s>0$. Indeed, looking to Proposition 13 and Remark 14a), we claim that the conclusions of Theorem 16 B) hold for $s \geq 3$ (and those of Theorem [16A) for $s \geq 0$, see Example 35] in the next section). Indeed we need only to verify its assumptions. Since $C$ is smooth and $\theta_{C}:=\widetilde{K}_{B}^{*}$ is of degree $\operatorname{deg} \theta_{C}=-\operatorname{deg} \widetilde{K}_{B}=2-2 g(g=$ the genus of $C)$, we get $\mathrm{H}^{1}\left(\theta_{C}(v)\right)=0$ and $h^{0}\left(\theta_{C}(v)\right)=d v+3-3 g=4 v+3$ for $v \geq 0$ (37, Lect. 11). Since we have an exact sequence

$$
\left.0 \rightarrow \theta_{C} \rightarrow \theta_{\mathbb{P}}\right|_{C} \rightarrow \mathcal{N}_{C} \rightarrow 0
$$

where $\left.\theta_{\mathbb{P}}\right|_{C}=\operatorname{coker}\left(\mathcal{O}_{C} \rightarrow \mathcal{O}_{C}(1)^{\oplus 5}\right)$ is the tangent sheaf of $\mathbb{P}=\mathbb{P}^{4}$ restricted to $C$, we get ${ }_{0} \operatorname{Ext}_{B}^{1}\left(I_{B} / I_{B}^{2}, B\right)=0$ from $\mathrm{H}^{1}\left(\mathcal{N}_{C}\right)=0$ and (15). Moreover,

$$
{ }_{v} \operatorname{Ext}_{B}^{1}\left(S_{2}\left(K_{B}\right), K_{B}\right)^{\vee} \simeq{ }_{-v} \mathrm{H}_{\mathfrak{m}}^{1}\left(S_{2}\left(K_{B}\right)\right)
$$


and since $\mathrm{H}_{*}^{0}\left(C, \widetilde{S_{2}\left(K_{B}\right)}\right) \rightarrow \mathrm{H}_{\mathfrak{m}}^{1}\left(S_{2}\left(K_{B}\right)\right)$ is surjective and

$$
\mathrm{H}^{0}\left(C, \widetilde{S_{2}\left(K_{B}\right)}(-v)\right)^{\vee} \simeq \mathrm{H}^{1}\left(\theta_{C}(v)\right),
$$

we get

$$
{ }_{v} \operatorname{Ext}_{B}^{1}\left(S_{2}\left(K_{B}\right), K_{B}\right)=0 \quad \text { for } \quad v \geq 0 .
$$

By Remark 14a), we know ${ }_{-s} \operatorname{Ext}_{B}^{1}\left(I_{B} / I_{B}^{2}, K_{B}\right)=0$ for $s>2$, and the claim is proved for $s \geq 3$. In conclusion the quotient $A$ is an unobstructed Gorenstein algebra for $s \geq 3$ and

$$
\begin{aligned}
\operatorname{dim}_{(A)} \operatorname{GradAlg}^{H_{A}}(R) & =h^{0}\left(\mathcal{N}_{C}\right)+h^{0}\left(\theta_{C}(s)\right)-1 \\
& =5 d+1-g+d s+3-3 g-1=4 s+23 .
\end{aligned}
$$

Remark 18. This example shows that if $\operatorname{Proj}(B)$ is a smooth curve of degree $d$ and genus $g$, and if we let $v$ be such that $2-2 g+v d>2 g-2$, i.e. $v>(4 g-4) / d$, then $\mathrm{H}^{1}\left(\theta_{C}(v)\right)=0$ and we get both ${ }_{v} \operatorname{Ext}_{B}^{1}\left(S_{2}\left(K_{B}\right), K_{B}\right)=0$ and $\operatorname{dim}\left(K_{B}^{*}\right)_{v}=$ $h^{0}\left(\theta_{C}(v)\right)=d v+3-3 g$. In particular if $s>\max \left\{2 \operatorname{reg}\left(I_{B}\right)-2,(4 g-4) / d\right\}$ and $B$ is unobstructed, then $A$ is unobstructed and $H_{B}$-generic by Proposition [13B) and Remark 14a) and

$$
\operatorname{dim}_{(A)} \operatorname{GradAlg}^{H_{A}}(R)=\operatorname{dim}\left(N_{B}\right)_{0}+d s+2-3 g .
$$

Hence we may replace " $s>>0$ " in Theorems 1 and 16 by " $s>\max \left\{2 \operatorname{reg}\left(I_{B}\right)-\right.$ $2,(4 g-4) / d\} "$ in this case.

Now we consider Artinian Gorenstein quotients. In particular we will concentrate on Artinian reductions obtained from algebras $A$ similar to those in Example 17 through modding out by a general linear form $L$ (cf. Example 21 below). More generally, let $B \simeq R / I_{B}$ be an $n$-dimensional Cohen-Macaulay algebra, let $A \simeq$ $B / K_{B}(-s)$, and let $R_{L}:=R /(L) \rightarrow B_{L}=B /(L) \rightarrow A_{L}=A /(L)$ be their quotients modulo $(L)$. The Hilbert functions of $A_{L}$ and $B_{L}$ are given by the first differences of the Hilbert functions of $A$ and $B$, respectively, e.g. $H_{B_{L}}(v)=H_{B}(v)-H_{B}(v-1)=$ $\Delta H_{B}(v)$. Moreover an $R_{L}$-free minimal resolution of $B_{L}$ has the same graded Betti numbers as the $R$-free minimal resolution of $B$ (34, p. 28), i.e. their numbers $n_{j, i}$ given in (6) coincide. Dualizing (6) we see that the minimal resolutions of $K_{B}(n+c)$ and $K_{B_{L}}(n+c-1)$ also coincide, i.e. $K_{B} \otimes B_{L} \simeq K_{B_{L}}(-1)$. Looking to (13) it follows that $A_{L}$ is given by

$$
0 \rightarrow K_{B_{L}}(-s-1) \rightarrow B_{L} \rightarrow A_{L} \rightarrow 0
$$

(note $-s-1$ instead of $-s !$ ) and $A_{L}$ is a Gorenstein quotient, well defined by a twist of the canonical module. Hence we may apply Proposition 13 and Theorem[16. To compute $\operatorname{dim}\left(K_{B_{L}}^{*}\right)_{s+1}$ and verify its assumptions, we see, thanks to the arguments of Remark $14 \mathrm{a}$ ), that $\delta\left(B_{L}\right)_{-s-1}=0$ and ${ }_{-s-1} \operatorname{Ext}_{B_{L}}^{1}\left(I_{B_{L}} / I_{B_{L}}^{2}, K_{B_{L}}\right)=0$ (where $\left.I_{B_{L}}=\operatorname{ker}\left(R_{L} \rightarrow B_{L}\right)\right)$, provided $s+1>\max n_{c, i}+\max n_{2, i}-(n-1)-c$, which is equivalent to the inequality of that remark, used to show $\delta(B)_{-s}=0$. To check the other assumptions, we claim that

Lemma 19. (i) If ${ }_{v-1} \operatorname{Ext}_{B}^{1}\left(S_{2}\left(K_{B}\right), K_{B}\right)=0$, then $\operatorname{dim}\left(K_{B_{L}}^{*}\right)_{v+1}=\operatorname{dim}\left(K_{B}^{*}\right)_{v}-$ $\operatorname{dim}\left(K_{B}^{*}\right)_{v-1}$.

(ii) If ${ }_{v} \operatorname{Ext}_{B}^{1}\left(S_{2}\left(K_{B}\right), K_{B}\right)={ }_{v-1} \operatorname{Ext}_{B}^{2}\left(S_{2}\left(K_{B}\right), K_{B}\right)=0$, then

$$
{ }_{v+1} \operatorname{Ext}_{B_{L}}^{1}\left(S_{2}\left(K_{B_{L}}\right), K_{B_{L}}\right)=0 .
$$


(iii) If $B$ is an unobstructed graded R-algebra and the $\operatorname{map}{ }_{-1} \operatorname{Ext}_{B}^{1}\left(I_{B} / I_{B}^{2}, B\right) \hookrightarrow$ ${ }_{0} \operatorname{Ext}_{B}^{1}\left(I_{B} / I_{B}^{2}, B\right)$ induced by $L$ is injective, then $B_{L}$ is an unobstructed graded $R_{L^{-}}$ algebra and

$$
\operatorname{dim}\left(N_{B_{L}}\right)_{0}=\operatorname{dim}\left(N_{B}\right)_{0}-\operatorname{dim}\left(N_{B}\right)_{-1} .
$$
$=0$.

(iv) If ${ }_{0} \operatorname{Ext}_{B}^{1}\left(I_{B} / I_{B}^{2}, B\right)={ }_{-1} \operatorname{Ext}_{B}^{2}\left(I_{B} / I_{B}^{2}, B\right)=0$, then ${ }_{0} \operatorname{Ext}_{B_{L}}^{1}\left(I_{B_{L}} / I_{B_{L}}^{2}, B_{L}\right)$

Proof. (i) and (ii). Apply $\operatorname{Hom}_{B}\left(S_{2}\left(K_{B}\right),-\right)$ to the exact sequence $0 \rightarrow K_{B} \rightarrow$ $K_{B}(1) \rightarrow K_{B_{L}} \rightarrow 0$ and use that $S_{2}\left(K_{B}\right) \otimes B_{L} \simeq S_{2}\left(K_{B_{L}}\right)(-2)$ and that $K_{B}^{*}=$ $\operatorname{Hom}_{B}\left(S_{2}\left(K_{B}\right), K_{B}\right)$.

(iii) The unobstructedness is just a very particular case of Theorem $5 \mathrm{~B}$ ) and Remark 6b) letting $I_{A / B} \simeq B(-1)$. To compute $N_{B_{L}}$, just apply ${ }_{0} \operatorname{Hom}_{B}\left(I_{B} / I_{B}^{2},-\right)$ to $0 \rightarrow B(-1) \rightarrow B \rightarrow B_{L} \rightarrow 0$. Continuing this sequence into a long exact sequence of Ext-groups, we prove (iv) as well because ${ }_{0} \operatorname{Ext}_{B_{L}}^{1}\left(I_{B_{L}} / I_{B_{L}}^{2}, B_{L}\right) \simeq$ ${ }_{0} \operatorname{Ext}_{B}^{1}\left(I_{B} / I_{B}^{2}, B_{L}\right)$.

Remark 20. a) In the case that $\operatorname{Proj}(B)$ is a curve $(n=2)$, we have by duality ${ }_{v} \operatorname{Ext}_{B}^{2}\left(S_{2}\left(K_{B}\right), K_{B}\right)^{\vee} \simeq{ }_{-v} \mathrm{H}_{\mathfrak{m}}^{0}\left(S_{2}\left(K_{B}\right)\right) \hookrightarrow S_{2}\left(K_{B}\right)_{-v}$. By Remark 14a) there is a surjection $S_{2}\left(\otimes R\left(n_{c, i}\right)\right) \rightarrow S_{2}\left(K_{B}(n+c)\right)$. Hence $S_{2}\left(K_{B}\right)_{-v}$ vanishes if $v>$ $2 \max n_{c, i}-2(n+c)$, i.e.

$$
{ }_{v} \operatorname{Ext}_{B}^{2}\left(S_{2}\left(K_{B}\right), K_{B}\right)=0 \quad \text { provided } \quad v>2 \operatorname{reg}\left(I_{B}\right)-2 n-2=2 \operatorname{reg}\left(I_{B}\right)-6 .
$$

b) If the curve $C=\operatorname{Proj}(B)$ is a local complete intersection, then (15) shows that ${ }_{-1} \operatorname{Ext}_{B}^{2}\left(I_{B} / I_{B}^{2}, B\right)=0$ provided $\mathrm{H}^{1}\left(\mathcal{O}_{C}\left(n_{1, i}-1\right)\right) \simeq \mathrm{H}^{0}\left(\widetilde{K}_{B}\left(-n_{1, i}+1\right)\right)^{\vee}=0$, i.e.

$$
{ }_{-1} \operatorname{Ext}_{B}^{2}\left(I_{B} / I_{B}^{2}, B\right)=0 \quad \text { provided } \min n_{1, i}>\operatorname{reg}\left(I_{B}\right)-n=\operatorname{reg}\left(I_{B}\right)-2 .
$$

Example 21. We consider the Artinian reduction $A_{L}$ of the Gorenstein algebra of Example 17. Since $B$ is 2-dimensional we get the vanishing of ${ }_{0} \operatorname{Ext}_{B_{L}}^{1}\left(I_{B_{L}} / I_{B_{L}}^{2}, B_{L}\right)$, and hence the unobstructedness of $B_{L}$, from Lemma 19(iii) and Remark 20b). Moreover, since we have seen that Remark 14a) also applies to the Artinian reduction, we get ${ }_{-s-1} \operatorname{Ext}_{B_{L}}^{1}\left(I_{B_{L}} / I_{B_{L}}^{2}, K_{B_{L}}\right)=0$ for $s>2$. Finally Lemma 19(ii) and Remark 20k) show that ${ }_{v+1} \operatorname{Ext}_{B_{L}}^{1}\left(S_{2}\left(K_{B_{L}}\right), K_{B_{L}}\right)=0$ for $v \geq 0$. By Remark 14b) all assumptions of Proposition $13 \mathrm{~B}$ ) concerning $B_{L}$ are satisfied. It follows that $A_{L}$ is an unobstructed Artinian Gorenstein algebra for $s \geq 3$, i.e. $\operatorname{PGor}\left(H_{L}\right)$ is smooth at $\left(A_{L}\right)$ by Theorem 11 and

$$
\operatorname{dim}_{\left(A_{L}\right)} \operatorname{PGor}\left(H_{L}\right)=\operatorname{dim}\left(N_{B_{L}}\right)_{0}+\operatorname{dim}\left(K_{B_{L}}^{*}\right)_{s+1}-1=3 d+d-1=15 .
$$

Note that the Hilbert function of $B_{L}$ is given by $(1,4,4,4, \ldots)$. Since the $h$-vector of $A_{L}$ is the $(s+2)$-tuple $(1,4,4, \ldots, 4,1)$, we get that the value of $\operatorname{dim}_{\left(A_{L}\right)} \operatorname{PGor}\left(H_{L}\right)$ above coincides with the dimension given in [21, Thm. 2.6 and Thm. 4.10A.

Finally we claim that this conclusion about $A_{L}$ holds for any Artinian Gorenstein quotient $A$ (well-defined by a twist of the canonical module of $B$ ) with the same graded Betti numbers as $A_{L}$. Indeed to use Remark [14a) we only need the graded Betti numbers of the minimal resolution. Since we may prove Remark 20 for a one-dimensional $B$ by replacing $\operatorname{Ext}_{B}^{2}(-,-)$ by the corresponding $\operatorname{Ext}_{B}^{1}(-,-)$ (in b) we use ${ }_{0} \operatorname{Ext}_{B}^{1}\left(I_{B} / I_{B}^{2}, B\right) \simeq{ }_{0} \operatorname{Ext}_{B}^{1}\left(I_{B} \otimes_{R} K_{B}, K_{B}\right) \simeq{ }_{0} \mathrm{H}_{\mathfrak{m}}^{0}\left(I_{B} \otimes_{R} K_{B}\right)^{\vee}$ in order to see that this group vanishes for $\left.\min n_{1, i}>\operatorname{reg}\left(I_{B}\right)-1\right)$, we get the claim. 
Remark 22. Pushing the arguments of this example a little further, we get that if $Y=\operatorname{Proj}(B)$ is a zero-scheme of degree $d$ and if $v \geq 2 \operatorname{reg}\left(I_{B}\right)-3$, then ${ }_{v} \operatorname{Ext}_{B}^{1}\left(S_{2}\left(K_{B}\right), K_{B}\right)=0$ and $\operatorname{dim}\left(K_{B}^{*}\right)_{v}=d$. Indeed with $v$ as above we have $S_{2}\left(K_{B}\right)_{-v}=0$ and ${ }_{-v} \mathrm{H}_{\mathfrak{m}}^{0}\left(S_{2}\left(K_{B}\right)\right)=0$ (cf. Remark 20k) ) and it follows that ${ }_{v} \operatorname{Ext}_{B}^{1}\left(S_{2}\left(K_{B}\right), K_{B}\right)=0$ by Gorenstein duality. Hence it suffices to show $\left(K_{B}^{*}\right)_{v} \simeq$ $\mathrm{H}^{0}\left(Y, \widehat{K_{B}^{*}}(v)\right)$, i.e. to show ${ }_{v} \mathrm{H}_{\mathfrak{m}}^{1}\left(K_{B}^{*}\right)=0$. By Gorenstein duality this group is dual to ${ }_{-v} \operatorname{Hom}_{B}\left(K_{B}^{*}, K_{B}\right)={ }_{-v} \operatorname{Hom}_{B}\left(\operatorname{Hom}_{B}\left(S_{2}\left(K_{B}\right), K_{B}\right), K_{B}\right) \simeq S_{2}\left(K_{B}\right)_{-v}=0$. In this case note that $\left(K_{B}^{*}\right)_{v}$ contains a regular section. In particular, if $s \geq 2 \operatorname{reg}\left(I_{B}\right)$ and $B$ is unobstructed, we have by Proposition [13 $\mathrm{B}$ ) and Remark [14 $\mathrm{a}$ ) that $A$ is unobstructed and $H_{B}$-generic and that

$$
\operatorname{dim}_{(A)} \operatorname{PGor}\left(H_{A}\right)=\operatorname{dim}_{(A)} \operatorname{GradAlg}^{H_{A}}(R)=\operatorname{dim}\left(N_{B}\right)_{0}+d-1 ;
$$

cf. Theorem 11. Hence we may replace " $s>>0$ " in Theorem1 (and in Theorem 16 provided $B$ is generically syzygetic) by " $s \geq 2 \operatorname{reg}\left(I_{B}\right)$ ".

Now we will study the case $s>>0$ of Theorem [16 to see that (13) actually determines a well-defined injective application from the set of irreducible components of $\operatorname{GradAlg}^{H_{B}}(R)$ containing locally licci, Cohen-Macaulay codimension $c$ quotients of $R$ (not necessarily unobstructed!), to the set of irreducible components of $\operatorname{GradAlg}^{H_{A}}(R)$ containing Gorenstein codimension $c+1$ quotients of $R$. To be precise let $U_{s} \subset \operatorname{GradAlg}^{H^{\prime}}(R)$ be an open (e.g. the largest open) subscheme whose corresponding k-points $(R \rightarrow B)$ are such that $B$ is CohenMacaulay and generically Gorenstein, $\operatorname{Proj}(B)$ is locally licci and satisfies $H_{B}=H^{\prime}$, ${ }_{s} \operatorname{Ext}_{B}^{1}\left(S_{2}\left(K_{B}\right), K_{B}\right)=0$ and $\left(K_{B}^{*}\right)_{s}$ contains a regular section. When we apply our results we usually take $U_{s}$ to be the open set in which " $B$ is generically Gorenstein and $\operatorname{Proj}(B)$ is locally licci" is replaced by "Proj $(B)$ is locally a complete intersection" or "Proj $(B)$ is smooth" or "Proj $(B)$ is locally Gorenstein and the codimension of $B$ is $c=3$ ", and with $s$ so large such that every such Cohen-Macaulay quotient $B$ satisfies ${ }_{s} \operatorname{Ext}_{B}^{1}\left(S_{2}\left(K_{B}\right), K_{B}\right)=0$ and $\left(K_{B}^{*}\right)_{s}$ contains a regular section (cf. Remarks 18 and 22). If we take a point $(R \rightarrow B)$ of $U_{s}$ and two particular quotients $B \rightarrow A_{1}$ and $B \rightarrow A_{2}$ (corresponding to two regular sections $\sigma_{i}$ of $\left(K_{B}^{*}\right)_{s}$ ), we have $I_{A_{1} / B} \simeq I_{A_{2} / B}$ as graded $B$-modules. Recalling that the ${ }_{0} \mathrm{H}^{2}(B, A, A)$-group of Lemma 7 is given by ${ }_{s} \operatorname{Ext}_{B}^{1}\left(S_{2}\left(K_{B}\right), K_{B}\right.$ ) (Lemma 15), we can take the open set $U$ of Lemma 7 to contain $\left(B \rightarrow A_{2}\right)$ provided it contains $\left(B \rightarrow A_{1}\right)$. Hence we can let $U$ be $q^{-1}\left(U_{s}\right)$ intersected by the space of those quotients $(B \rightarrow A)$ which correspond to the regular sections of $\left(K_{B}^{*}\right)_{s}$, where $q: \operatorname{GradAlg}\left(H_{B}, H_{A}\right) \rightarrow \operatorname{GradAlg}^{H_{B}}(R)$ is the first projection. Calling this intersection $q^{-1}\left(U_{s}\right)_{r e g}$, we have an incidence correspondence (cf. the text before (10)

$$
q^{-1}\left(U_{s}\right)_{\text {reg }} \stackrel{q_{\text {res }}}{\longrightarrow} U_{s} \subset \operatorname{GradAlg}^{H_{B}}(R)
$$

$$
\begin{gathered}
\downarrow^{p_{\text {res }}} \\
\operatorname{GradAlg}^{H_{A}}(R)
\end{gathered}
$$

of restricted projection morphisms in which $q_{r e s}$ and $p_{r e s}$ have nice properties. Note that $v=n-\operatorname{reg}\left(I_{B}\right), n=\operatorname{dim} B$, is the largest integer such that $\left(K_{B}\right)_{v}=0$ by Remark 14a). Letting $n_{j, i}$ be the number appearing in the minimal resolution (6) of $B$, we get the following proposition. 
Proposition 23. With notations as above, we have

i) The morphism $q_{\text {res }}$ in (18) is smooth and its fibers are geometrically connected, of fiber dimension $\operatorname{dim}\left(K_{B}^{*}\right)_{s}-1$.

ii) If $U_{n_{1 i}}$ is the open subset of $U_{s}$ whose corresponding k-points $(B)$ satisfy $\max n_{1, i} \leq n-\operatorname{reg}\left(I_{B}\right)+s$, then $p^{-1}\left(p_{\text {res }}\left(q_{\text {res }}^{-1}\left(U_{n_{1 i}}\right)\right)\right)=q_{\text {res }}^{-1}\left(U_{n_{1 i}}\right)$ and $p_{\text {res }}$ is unramified and injective on $q_{r e s}^{-1}\left(U_{n_{1 i}}\right)$. Moreover if $s \geq 2 \operatorname{reg}\left(I_{B}\right)-n$, then $U_{n_{1 i}}=$ $U_{s}$.

iii) If $U_{n_{2 i}}$ is the open subset of $U_{s}$ whose k-points $(B)$ satisfy $\max n_{2, i} \leq$ $n-\operatorname{reg}\left(I_{B}\right)+s$, then $p^{-1}\left(p_{\text {res }}\left(q_{\text {res }}^{-1}\left(U_{n_{2 i}}\right)\right)\right)=q_{\text {res }}^{-1}\left(U_{n_{2 i}}\right)$ and the restriction of $p_{\text {res }}$ to $q_{\text {res }}^{-1}\left(U_{n_{2 i}}\right)$ is an isomorphism onto an open subscheme of $\operatorname{GradAlg}^{H_{A}}(R)$. In particular if $s \geq 2 \operatorname{reg}\left(I_{B}\right)-n+1$, then $U_{n_{2 i}}=U_{s}$ and the morphism pres in (18) is an isomorphism onto an open subscheme of $\operatorname{GradAlg}^{H_{A}}(R)$.

Proof. i) To see the smoothness of $q_{r e s}$, we combine Proposition 4(i) and Lemma 15 (see also the proof of Proposition 131). To show that the fibers of $q_{\text {res }}$ are (geometrically) connected, it suffices to see that two quotients $B \rightarrow A_{1}$ and $B \rightarrow A_{2}$, given as in (13) by two regular sections $\sigma_{i}$ of $\left(K_{B}^{*}\right)_{s}$, correspond to two $k$-points of a flat irreducible family $U^{\prime} \subset \mathbb{A}^{1}(k)=\operatorname{Spec}(k[t])$ contained in the fiber $q_{r e s}^{-1}((B))$. This is, however, quite trivial because we can easily show that $t \sigma_{1}+(1-t) \sigma_{2}$ is a regular section of $\left(K_{B[t]}^{*}\right)_{s}$ for any $t$ in some open set of $\mathbb{A}^{1}(k)$ containing $t=0$ and $t=1$.

ii) It is straightforward to get ii) from Lemma 7b) because $K_{B}(-s)_{\max n_{1, i}}=0$ by assumption.

iii) Note that $p$ is universally injective ("radiciel") on the set $U$ of Lemma 7 because the proof of the injectivity of $\left.p\right|_{U}$ in Lemma $7 \mathrm{~b}$ ) extends to $F$-valued points where $F$ is any field extension of $k$. Hence by [13, Thm.17.9.1, it suffices to prove that $p_{\text {res }}$ is etale on $q_{\text {res }}^{-1}\left(U_{n_{2 i}}\right)$. By (ii) above, it suffices to prove smoothness. Looking to Remark $14 \mathrm{a}$ ), we see that ${ }_{-s} \operatorname{Ext}_{B}^{1}\left(I_{B} / I_{B}^{2}, K_{B}\right)=0$ by assumption. The first sentence of the proof of Theorem[16 shows that $I_{B}$ is generically syzygetic. Hence we get the smoothness of $p_{\text {res }}$ by Proposition 4 (ii) and the proof of Theorem $5 \mathrm{~B}$ ).

Recall that a quotient $A$ of $R$ has the Weak Lefschetz property if there is a linear form $l$ and an Artinian reduction $A_{L}:=A /(L)$ of $A$ such that the multiplication $l:\left(A_{L}\right)_{v} \rightarrow\left(A_{L}\right)_{v+1}$ is either injective or surjective for every $v$ (cf. the text before (17)). Letting "general member or $k$-point" mean "any member or $k$-point in a suitable open dense subset", we get

Theorem 24. Let $s \geq 2 \operatorname{reg}\left(I_{B}\right)-n+1$. Then the correspondence (18) determines a well-defined injective application $\pi$ from the set of irreducible components $W$ of $U_{s}$ (whose members $B$ are codimension c Cohen-Macaulay quotients of $R$ satisfying the defining conditions of $U_{s}$ above) to the set of irreducible components $V$ of $\operatorname{GradAlg}^{H_{A}}(R)$ whose general members are Gorenstein codimension $c+1$ quotients $A$ of $R$ satisfying the Weak Lefschetz property. In this application the generically smooth components correspond. Indeed if $m_{W}$ (resp. $m_{V}$ ) is the maximal ideal of the local ring of a general k-point of $W$ (resp. of $V=\pi(W)$ ), then $\operatorname{dim} m_{V} / m_{V}^{2}-\operatorname{dim} V=\operatorname{dim} m_{W} / m_{W}^{2}-\operatorname{dim} W$, and we have

$$
\operatorname{dim} V=\operatorname{dim} W+\operatorname{dim}\left(K_{B}^{*}\right)_{s}-1 .
$$

Proof. By Proposition 23(i) and [17, Prop. 1.8, $q_{\text {res }}^{-1}(W)$ is an irreducible component of $q^{-1}\left(U_{s}\right)_{\text {reg }}$. The application is therefore well-defined, and it is injective by 
Proposition 23(iii). Since $q_{r e s}$ is smooth and $p_{r e s}$ is an open embedding, we easily get the dimension formulas.

It remains to see that $A$ has the Weak Lefschetz property. Indeed we claim that any $A^{\prime}$ which corresponds to a point in the image $p_{r e s}\left(q^{-1}\left(U_{s}\right)_{r e g}\right)$ has the Weak Lefschetz property. First suppose $\operatorname{dim} B^{\prime}=1$, with $\left(B^{\prime} \rightarrow A^{\prime}\right) \in q^{-1}\left(U_{s}\right)_{\text {reg }}$. Since $\operatorname{reg}\left(I_{B^{\prime}}\right)=\sigma\left(B^{\prime}\right)$ where $\sigma\left(B^{\prime}\right)$ is defined by $\min \left\{i \mid \Delta H_{B^{\prime}}(i)=0\right\}$ (e.g. [35], Sect. 2), it follows that the multiplication by a regular element of $B^{\prime}$ of degree one induces a bijection $\left(B^{\prime}\right)_{t-1} \rightarrow\left(B^{\prime}\right)_{t}$ for $t \geq \operatorname{reg}\left(I_{B^{\prime}}\right)$, hence $\left(A^{\prime}\right)_{t-1} \rightarrow\left(A^{\prime}\right)_{t}$ is surjective for $t \geq \operatorname{reg}\left(I_{B^{\prime}}\right)$. Let $t<\operatorname{reg}\left(I_{B^{\prime}}\right)$. Since the assumption $s \geq 2 \operatorname{reg}\left(I_{B}\right)-n+1$ leads to $K_{B^{\prime}}(-s)_{v}=0$ for $v \leq \operatorname{reg}\left(I_{B^{\prime}}\right)+1$, we get $\left(B^{\prime}\right)_{v} \simeq\left(A^{\prime}\right)_{v}$ for $v \leq \operatorname{reg}\left(I_{B^{\prime}}\right)+1$ and certainly for $v<\operatorname{reg}\left(I_{B^{\prime}}\right)$. The latter is precisely what we need to get the injectivity of $\left(A^{\prime}\right)_{t-1} \rightarrow\left(A^{\prime}\right)_{t}$ from the injectivity of $\left(B^{\prime}\right)_{t-1} \rightarrow\left(B^{\prime}\right)_{t}$. Hence $A^{\prime}$ has the Weak Lefschetz property. Moreover if $n=\operatorname{dim} B^{\prime}>1$ we know that the graded Betti numbers of $B^{\prime}$ and of $K_{B^{\prime}}(-n)$ are unchanged by modding out by a $(n-1)$-dimensional space $L^{\prime}$ of general linear forms (cf. the explanation to (17)). Hence we may apply the argument above to the one-dimensional quotient $B^{\prime} /\left(L^{\prime}\right)$, and we are done.

Remark 25. a) Let $\operatorname{dim} B=1$, i.e. let $\operatorname{Proj}(B)$ be a zero-scheme of degree $d$ and let $s \geq 2 \operatorname{reg}\left(I_{B}\right)$. Then we automatically get the vanishing of the Ext $^{1}$-group appearing in the definition of $U_{s}$ by Remark 22. Since the quotients of $U_{s}$ satisfy $K_{B}(-s)_{\operatorname{reg}\left(I_{B}\right)+1}=0$, we get $\operatorname{dim} B_{\operatorname{reg}\left(I_{B}\right)+1}=\operatorname{dim} A_{\operatorname{reg}\left(I_{B}\right)+1}$. Hence we see that the Hilbert function of each $A$ in Theorem 24 is an unimodal SI-sequence with at least three consecutive peaks, that is, $H_{A} \supset(d, d, d)$ (because $A$ has the Weak Lefschetz property; see e.g. [35]). Moreover,

$$
\operatorname{dim} V=\operatorname{dim} W+d-1 .
$$

Indeed by Proposition 23)(iii) we have much more, namely a smooth morphism $q_{\text {res }} \circ p_{\text {res }}^{-1}$ of schemes defined on $p_{\text {res }}\left(q^{-1}\left(U_{s}\right)_{\text {reg }}\right)$ with values in $\operatorname{GradAlg}^{H_{B}}(R)$, allowing us to compare properties of $\operatorname{GradAlg}^{H_{B}}(R)$ at $(B)$ with the corresponding properties of $\operatorname{GradAlg}^{H_{A}}(R)$ at $(A)$ beyond that done in Theorem 24, Note that this correspondence is well studied by Iarrobino-Kanev if the codimension of $B$ is $c=2$ ([21], Sect. 5.3.2), in which case they get a topological fibration defined on the whole parameter space PGor $\left(H_{A}\right)$. In that case they also get the dimension formula above $\left(21\right.$, Cor. 5.50). Since the points of $p_{r e s}\left(q^{-1}\left(U_{s}\right)_{r e g}\right) \subset \operatorname{PGor}\left(H_{A}\right)$ satisfy the Weak Lefschetz property, we cannot in general extend the morphism $q_{\text {res }} \circ p_{\text {res }}^{-1}$ to the whole of PGor $\left(H_{A}\right)$ (cf. [3])!

b) Let $\operatorname{dim} B=1$ and suppose $s \geq 2 \operatorname{reg}\left(I_{B}\right)-1$. Using the arguments of a) it follows that the $h$-vector of $A$ is an SI-sequence with at least two peaks. By Proposition 23ii) the correspondence (18) applies provided the points $(B)$ of $U_{s}$ (or of $U_{n_{1 i}}$ if we only assume $\left.\max n_{1, i} \leq 1-\operatorname{reg}\left(I_{B}\right)+s\right)$ ) satisfy the assumptions of Proposition [13A). These assumptions are satisfied if $B$ is licci (Remark 14k)) or if $I_{B}$ is generically syzygetic (e.g. if $c \leq 3$ ) and satisfies

$$
\left(\mathrm{H}_{2}(R, B, B) \otimes_{B} A\right)_{s}=0 \text { and }{ }_{0} \mathrm{H}_{\mathfrak{m}}^{0}\left(I_{B} \otimes_{R} K_{B}\right)=0
$$

(Remark 140) because the latter vanishing is equivalent to ${ }_{0} \operatorname{Ext}_{B}^{1}\left(I_{B} / I_{B}^{2}, B\right)=0$ by Gorenstein duality. Hence we get by Proposition [13A) and Theorem 11 that to each irreducible component $W$ of say $U_{s}$ whose general member is licci or as above, then (18) determines an irreducible closed subset $V$ of codimension 
${ }_{-s} \operatorname{ext}_{B}^{1}\left(I_{B} / I_{B}^{2}, K_{B}\right)$ in PGor $\left(H_{A}\right)$ such that PGor $\left(H_{A}\right)$ is generically smooth along $V$, and $\operatorname{dim} V=\operatorname{dim} W+d-1$. If $c=2$ this result is known (21], Sect. 5.3.3 and Cor. 5.50).

Example 26. Here we will use Theorem 24 and Proposition 8 to give classes of examples in which the scheme $\operatorname{GradAlg}^{H}(R)$ has several irreducible components. To simplify the analysis, we will use the classification of ACM (arithmetically CohenMacaulay) curves on a general rational ACM surface appearing in 29]. So our examples will yield irreducible components of $\operatorname{GradAlg}^{H}(R)$ of Gorenstein codimension 4 quotients of dimension one. It is not obvious that the corresponding Artinian reductions lead to irreducible components of PGor $(H)$ of Artinian Gorenstein codimension 4 quotients.

First we explicitly describe three irreducible components of $\operatorname{Hilb}^{p}\left(\mathbb{P}^{4}\right)$ with the same Hilbert function (in fact their graded Betti numbers are the same, and are the minimum possible consistent with the Hilbert function). Recalling that a general Castelnuovo surface $F \subset \mathbb{P}^{4}$ is obtained by blowing up 8 general points in $\mathbb{P}^{2}$, thus that $\operatorname{Pic}(F) \simeq \mathbb{Z}^{9}$, we write the hyperplane section and the canonical divisor as $H=\left(4 ; 2,1^{7}\right)$ and $K=\left(-3 ;-1^{8}\right)$, respectively. Consider the three families of curves, $C \in\left|C_{0}+t H\right|$, of [29], p. 73, given by

$$
\left(4 t+1 ; 2 t, t^{7}\right),\left(4 t+2 ; 2 t, t+1^{4}, t^{3}\right),\left(4 t+3 ; 2 t, t+2, t+1^{6}\right)
$$

having $C_{0} \cdot K=(-3,-2,-1)$, respectively. We always have $\left(d_{0}, g_{0}\right):=\left(\operatorname{deg}\left(C_{0}\right)\right.$, $\left.\operatorname{gen}\left(C_{0}\right)\right)=(4,0)$ and $H^{2}=5$. By Proposition 8 any such $C$ is unobstructed provided $t \geq 3$ and

$$
\operatorname{dim}_{(C)} \operatorname{Hilb}^{p}\left(\mathbb{P}^{4}\right)=31-C_{0} \cdot K+\left(11 t+5 t^{2}\right) / 2,
$$

where $p$ is the Hilbert polynomial of $C$. Since the three families have different $C_{0} \cdot K$, they must correspond to three different components of $\mathrm{Hilb}^{p}\left(\mathbb{P}^{4}\right)$.

We claim that all curves $C=\operatorname{Proj}(B)$ of the three families have the same Hilbert function, and hence that their quotients $B$ belong to the same $\operatorname{GradAlg}^{H}(R)$. Indeed looking to the sequence

$$
0 \rightarrow \mathcal{J}_{C / F} \simeq \mathcal{O}_{F}(-C) \rightarrow \mathcal{O}_{F} \rightarrow \mathcal{O}_{C} \rightarrow 0
$$

and the corresponding one for $C_{0}$, we get $h^{1}\left(\mathcal{O}_{C}(v)\right)=h^{1}\left(\mathcal{O}_{C_{0}}(v-t)\right)=0$ for $v \geq t$. It is well known that the homogeneous ideal of $F$ has three minimal generators of degrees 2,3,3 and two relations of degrees 4,4. Combining $h^{0}\left(\mathcal{J}_{C / F}(v)\right)=h^{0}\left(\mathcal{J}_{C_{0} / F}(v-t)\right)$ and $s\left(C_{0} / F\right)=2$ with $h^{1}\left(\mathcal{O}_{C}(t)\right)=0$, we see that the additional minimal generators of $I_{B}$ sit all in degree $t+2$. Hence we get that all $C$ of the three families have the same Hilbert function and the same regularity, $\operatorname{reg}\left(I_{B}\right)=t+2$, i.e. we get the claim. Indeed the members of the three families satisfy $H_{B}(v)=h^{0}\left(\mathcal{O}_{C}(v)\right)=d v+1-g$ for $v \geq t$ and $H_{B}(v)=h^{0}\left(\mathcal{O}_{F}(v)\right)$ for $v<t$, and we easily find the minimal resolution to be

$0 \rightarrow R(-t-4)^{5} \rightarrow R(-t-3)^{10} \oplus R(-4)^{2} \rightarrow R(-t-2)^{5} \oplus R(-3)^{2} \oplus R(-2) \rightarrow I_{B} \rightarrow 0$.

Now, thanks to (9), the corresponding Cohen-Macaulay algebras $B$ are unobstructed as graded algebras and $\operatorname{dim}_{(B)} \operatorname{GradAlg}^{H}(R)=\operatorname{dim}_{(C)} \operatorname{Hilb}^{p}\left(\mathbb{P}^{4}\right)$. The three families must therefore correspond to three generically smooth irreducible components of $\operatorname{GradAlg}^{H_{B}}(R)$ of different dimensions whose general members $B$ are two-dimensional Cohen-Macaulay codimension 3 quotients of $R$. 
By Theorem 24, we get three generically smooth irreducible components $V$ of $\operatorname{GradAlg}^{H_{A}}(R)$ whose general members are one-dimensional Gorenstein codimension 4 quotients $A$ of $R$. Indeed Theorem 24 applies for $s>2 \operatorname{reg}\left(I_{B}\right)-\operatorname{dim} B=$ $2 t+2$ provided we can show ${ }_{s} \operatorname{Ext}_{B}^{1}\left(S_{2}\left(K_{B}\right), K_{B}\right)=0$ for such $s$. Looking to the arguments of Remark 18, it suffices to prove $s>(4 g-4) / d$ which is straightforward since $\left(d_{0}, g_{0}\right)=(4,0), d=d_{0}+5 t$ and $2 g=2 g_{0}+2 t d_{0}+5 t^{2}-3 t$ by the adjunction formula $2 g-2=C \cdot(C+K)$. Now, inserting these values for $d$ and $g$ into the expression $\operatorname{dim}\left(K_{B}^{*}\right)_{s}=d s+3-3 g$ of Remark [18, we get, for every $t \geq 3$ and every $s>2 t+2$, three irreducible components $V$ of $\operatorname{GradAlg}^{H_{A}}(R)$ satisfying

$\operatorname{dim} V=\operatorname{dim}_{C} \operatorname{Hilb}^{p}\left(\mathbb{P}^{4}\right)+\operatorname{dim}\left(K_{B}^{*}\right)_{s}-1=33-2 t-5 t^{2}-C_{0} \cdot K+s(4+5 t)$.

It is not difficult to compute the Hilbert function $H_{A}$ from the Hilbert function $H_{B}$. Indeed the $h$-vector of $A$, i.e. the $h$-vector of an Artinian reduction of $A$, is just

$$
(1,4,9,14,19, \ldots, 4+5 t, 4+5 t, 4+5 t, \ldots, 19,14,9,4,1) .
$$

The simplest case $t=3$ and $s=9$ yields the $h$-vector $(1,4,9,14,19,19,19,14,9,4,1)$.

Remark 27. We can use the classification of ACM curves on a general rational ACM surface in $\mathbb{P}^{4}$ in [29] to get many more reducible schemes $\operatorname{GradAlg}^{H_{A}}(R)$ whose general members are Gorenstein codimension 4 algebras. For instance on a Castelnuovo surface, look to the linear systems $C \in\left|C_{0}+t H\right|$ of [29], p. 73, where $C_{0}$ is a minimal curve and $t \geq 0$. For both $\left(d_{0}, g_{0}\right)=(5,1)$ and $(6,2)$ there are at least two linear systems with different $C_{0} \cdot K$. Here $s\left(C_{0} / F\right)=2$ and Proposition 8 applies for $t \geq 3$. Since $\mathrm{H}^{1}\left(\mathcal{O}_{C_{0}}(1)\right)=0$ one may see that $\operatorname{reg}\left(I_{B}\right)=t+3(C=\operatorname{Proj}(B))$ and that Theorem 24 applies for every $s>2 t+4$. The $h$-vectors of the simplest case $t=3$ and $s=11$ of the two families are

$$
(1,4,9,14,19,20,20,20,19,14,9,4,1) \text { and }(1,4,9,14,19,21,21,21,19,14,9,4,1) .
$$

For both $\left(d_{0}, g_{0}\right)=(2,0)$ and $(3,0)$ there are also two linear systems with different $C_{0} \cdot K$. Now $s\left(C_{0} / F\right)=1$ and $\mathrm{H}^{1}\left(\mathcal{O}_{C_{0}}\right)=0$ and Proposition 8 applies for $t \geq 4$ and Theorem 24 for $s>2 \operatorname{reg}\left(I_{B}\right)-2=2 t+2$. The $h$-vectors of the simplest case $t=4$ and $s=11$ are

$$
(1,4,9,14,19,22,22,22,19,14,9,4,1) \text { and }(1,4,9,14,19,23,23,23,19,14,9,4,1) .
$$

Putting the analysis on a Castelnuovo surface together we get that $\operatorname{GradAlg}{ }^{H_{A}}(R)$ has at least two generically smooth irreducible components whose general members are one-dimensional Gorenstein codimension 4 quotients $A$ of $R$ in the following range of the $h$-vector:

$$
(1,4,9,14, \ldots, 5 \alpha-1, \beta, \beta, \ldots, \beta, 5 \alpha-1,5 \alpha-6, \ldots, 9,4,1),
$$

where $5 \alpha-1 \leq \beta \leq 5 \alpha+4, \alpha \geq 3, \beta \geq 19$, and with at least three consecutive $\beta$ 's (three peaks).

Of course the part $(1,4,9,14, \ldots, 5 \alpha-1)$ of the $h$-vector comes from the Castelnuovo surface. On a cubic surface there are two linear systems with $\left(d_{0}, g_{0}\right)=(1,0)$ and with different $C_{0} \cdot K$, giving rise to reducible $\operatorname{GradAlg}^{H_{A}}(R)$ for every $t \geq 3$ and $s>2 t$. For $(t, s)=(3,7)$ the $h$-vector is $(1,4,7,10,10,10,7,4,1)$. Increasing $t$ to 4 and $s$ to 10 we get precisely the $h$-vector of Boij's example [3], which is different from ours because our algebras have the Weak Lefschetz property (see also [22], Thm. 3.9). There are also quite a lot of linear systems on a Bordiga surface 
with which we could make a similar analysis. All examples we get in this case have a symmetric $h$-vector starting with $(1,4,10,16,22, \ldots)$.

We end this section by using Theorem [16 to essentially reprove the smoothness and give a formula for the dimension of $\operatorname{GradAlg}^{H}(R)$ for (e.g. Artinian) Gorenstein codimension 3 quotients equivalent to those in [27, 28] or [6].

Example 28. Recall that if $A \simeq R / I_{A}$ is Gorenstein of codimension 3 with minimal resolution

$$
0 \rightarrow R(-f) \simeq F_{3} \rightarrow F_{2} \rightarrow F_{1} \rightarrow I_{A} \rightarrow 0 .
$$

One knows that $A$ is unobstructed and that $N_{A} \simeq \bigwedge^{2} I_{A}(f)(\operatorname{char}(k) \neq 2)$. It follows that

$$
\operatorname{dim}_{(A)} \operatorname{GradAlg}^{H_{A}}(R)=\operatorname{dim}\left(\bigwedge^{2} F_{1}\right)_{f}-\operatorname{dim}\left(F_{1} \otimes F_{2}\right)_{f}+\operatorname{dim}\left(S_{2}\left(F_{2}\right)\right)_{f} ;
$$

cf. 28, Thm. 2.6 and its Remark. In (20) it is not really necessary to suppose (19) to be minimal because we easily see that (20) is invariant under adding a common free factor to both $F_{1}$ and $F_{2}$.

If $B \simeq R / I_{B}$ is Cohen-Macaulay of codimension 2 (and hence licci), and $A$ is given as in Theorem 16, then we claim the dimension formula for

$$
\operatorname{dim}_{(A)} \operatorname{GradAlg}^{H_{A}}(R)
$$

in Theorem 16 coincides with (20). Note that since every irreducible component of the Hilbert scheme of Gorenstein codimension 3 quotients of $R$ contains a quotient constructed via (13) by [10, this example proves the generic smoothness of $\operatorname{GradAlg}^{H}(R)$ and PGor $(H)$, as well as (20). The argument holds in the case $\operatorname{char}(k)=2$ as well.

First we compute $\operatorname{dim}\left(K_{B}^{*}\right)_{s}$. Indeed the dual of (8) (for $c=2$ ) yields the exact sequence

$$
0 \rightarrow K_{B}^{*}(-n-c) \rightarrow G_{2} \otimes B \rightarrow G_{1} \otimes B \rightarrow I_{B} / I_{B}^{2} \rightarrow 0
$$

in which $\mathrm{H}_{1}$ is the kernel of $G_{1} \otimes B \rightarrow I_{B} / I_{B}^{2}$ by (4). By [2] one knows that

$$
0 \rightarrow \bigwedge^{i+1} G_{2} \rightarrow \bigwedge^{i+1} G_{1} \rightarrow \bigwedge^{i} G_{2} \rightarrow \mathrm{H}_{i} \rightarrow 0
$$

is exact for any $i$. Let $f=s+n+c$. Using the last-mentioned sequence for $i=1$, (21) and the $R$-free resolution of $G_{2} \otimes B$ which we easily deduce from (6), we get

$$
\operatorname{dim}\left(K_{B}^{*}\right)_{s}=\operatorname{dim}\left(\bigwedge^{2} G_{1}\right)_{f}-\operatorname{dim}\left(G_{1} \otimes G_{2}\right)_{f}+\operatorname{dim}\left(S_{2}\left(G_{2}\right)\right)_{f} .
$$

Next we will compute $\delta=\delta(B)_{-s}=\operatorname{dim}\left(G_{1}^{*} \otimes_{R} K_{B}\right)_{-s}-{ }_{-s} \operatorname{hom}_{B}\left(\mathrm{H}_{1}, K_{B}\right)$; cf. Remark 14d). If $r=\operatorname{rank} \mathrm{H}_{1}$, we have by (22) a resolution of $\mathrm{H}_{r-1}$ leading to

$$
\begin{aligned}
0 & \rightarrow G_{2}^{*} \rightarrow\left(\bigwedge^{2} G_{1}\right)^{*} \rightarrow\left(\bigwedge^{2} G_{2}\right)^{*} \rightarrow \mathrm{H}_{r-1} \otimes\left(\bigwedge^{r+1} G_{2}\right)^{*} \\
& \simeq \operatorname{Hom}\left(\mathrm{H}_{1}, K_{B}(n+c)\right) \rightarrow 0 .
\end{aligned}
$$

We have an $R$-free resolution of $K_{B}$ by dualizing (6) and hence a resolution of $G_{1}^{*} \otimes K_{B}$. Combining with (24), we get

$$
\begin{aligned}
-\delta=\operatorname{dim}\left(G_{1} \otimes G_{2}\right)_{-f}^{*}-\operatorname{dim}\left(S_{2}\left(G_{1}\right)^{*}\right)_{-f}-\operatorname{dim}\left(\bigwedge^{2} G_{2}\right)_{-f}^{*} & \\
& +\operatorname{dim}\left(G_{1}\right)_{-f}^{*}-\operatorname{dim}\left(G_{2}\right)_{-f}^{*} .
\end{aligned}
$$


Note that $\operatorname{dim}\left(N_{B}\right)_{0}$ is well known, e.g. one may easily deduce it from the exact sequence

$$
0 \rightarrow G_{1}^{*} \otimes G_{2} \rightarrow\left(\left(G_{1}^{*} \otimes G_{1}\right) \oplus\left(G_{2}^{*} \otimes G_{2}\right)\right) / k \rightarrow G_{2}^{*} \otimes G_{1} \rightarrow N_{B} \rightarrow 0
$$

of 30, Remark 16 ( $k$ is a 1-dimensional subspace). Putting these formulas together, using Theorem [16, we get exactly (20) because we find the following resolution of $I_{A}$ :

$$
0 \rightarrow R(-f) \rightarrow G_{1}^{*}(-f) \oplus G_{2} \rightarrow G_{2}^{*}(-f) \oplus G_{1} \rightarrow I_{A} \rightarrow 0
$$

by applying the mapping cone construction onto (13).

Inside the scheme $\mathrm{Z}(\mathrm{H})$ of Remark 10 we have the open subscheme ZGor $(H)$ consisting of not necessarily graded Gorenstein quotients $R \rightarrow A$ with Hilbert function $H$. ZGor $(H)$ has been studied in different contexts (see [21, p. 126, for references). Since by Remark $14 \mathrm{c}$ ) we easily see that ${ }_{v} \mathrm{H}^{2}(R, A, A)=0$ for $v \geq 0$ provided $B$ is licci, we can use Remark 10 to prove the smoothness and find the dimension of the scheme ZGor $(H)$ at $(A)$. Indeed ${ }_{v} \operatorname{hom}_{R}\left(I_{A}, A\right)$ is found using (10) as in the proof of Theorem 5. Also, if we apply ${ }_{v} \operatorname{Hom}\left(K_{B}(-s),-\right)$ to (13), we get ${ }_{v} \operatorname{hom}\left(K_{B}(-s), A\right)=\operatorname{dim}\left(K_{B}^{*}\right)_{s+v}-\operatorname{dim} B_{v}$. Since $A_{v}=0$ for $v>s$, we have

Proposition 29. Let $B \simeq R / I_{B}$ be licci and generically Gorenstein, and let $A$ be $a$ graded codimension one quotient of $B$, defined by (13) for some s. Then $\mathrm{ZGor}(H)$ is smooth at $(R \rightarrow A)$ and

$$
\operatorname{dim}_{(A)} \mathrm{Z}(\mathrm{H})=\sum_{v \geq 0}^{s}\left(\operatorname{dim}\left(N_{B}\right)_{v}-\delta(B)_{-s+v}\right)+\sum_{v \geq 0}^{s}\left(\operatorname{dim}\left(K_{B}^{*}\right)_{s+v}-\operatorname{dim} B_{v}\right) .
$$

Invoking Remark 14 ), we take the opportunity to state the main result of this section in the non-graded case. The proof is omitted because it is the same as in the graded case.

Theorem 30. Let $B \simeq R / I_{B}$ be Cohen-Macaulay, generically Gorenstein and generically syzygetic of dimension $\geq 1$ and suppose $B$ satisfies

$\operatorname{Ext}_{B}^{1}\left(I_{B} / I_{B}^{2}, B\right)=0, \quad \operatorname{Ext}_{B}^{1}\left(S_{2}\left(K_{B}\right), K_{B}\right)=0 \quad$ and $\quad \operatorname{Ext}_{B}^{2}\left(I_{B} / I_{B}^{2}, K_{B}\right)=0$.

Consider $K_{B}$ as an ideal of $B$ and let $A:=B / K_{B}$. Then $A$ is Gorenstein and strongly unobstructed in the sense $\mathrm{H}^{2}(R, A, A)=0$.

In particular let $B \simeq R / I_{B}$ be licci and generically Gorenstein. Then $A$ is Gorenstein and strongly unobstructed in the sense $\mathrm{H}^{2}(R, A, A)=0$. Indeed licci and generically Gorenstein imply Cohen-Macaulay and generically syzygetic as well as the vanishing of the three Ext-groups above.

\section{Computing the dimension via Liaison}

In this section we show how we can compute $\delta(B)_{-s}={ }_{-s} \operatorname{hom}_{R}\left(I_{B}, K_{B}\right)-$ ${ }_{-s} \operatorname{ext}_{B}^{1}\left(I_{B} / I_{B}^{2}, K_{B}\right)$ and $\operatorname{dim}\left(K_{B}^{*}\right)_{s}$. For instance it turns out that the difference $\operatorname{dim}\left(K_{B}^{*}\right)_{s}-\delta(B)_{-s}$ (which appears in Theorem 16) is quite easy to compute because, after adding a simple term to it, it becomes a liaison invariant among syzygetic ideals $I_{B}$. Moreover $\delta(B)_{-s}$ is computable in terms of $\operatorname{dim}\left(K_{B^{\prime}}^{*}\right)_{s}$ of the linked algebra $B^{\prime}$. To allow any complete intersection linkage of Cohen-Macaulay 
quotients and still get the liaison invariance described above, we need to also define $\delta(B)$ for Cohen-Macaulay quotients $R \rightarrow B$ which satisfy $\mathrm{H}_{2}(R, B, B) \neq 0$ generically. To do this we consider the exact sequence ([38, Sect. 2.1)

$$
0 \rightarrow \mathrm{H}_{2}(R, B, B) \rightarrow S_{2} I_{B} \rightarrow I_{B} \rightarrow I_{B} / I_{B}^{2} \rightarrow 0 .
$$

Let $\psi: \mathrm{H}_{\mathfrak{m}}^{n}\left(\mathrm{H}_{2}(R, B, B)\right) \rightarrow \mathrm{H}_{\mathfrak{m}}^{n}\left(S_{2} I_{B}\right), \operatorname{dim} B=n$, be the induced map.

Definition 31. Let $B=R / I_{B}$ be Cohen-Macaulay of dimension $n$, not necessarily generically syzygetic. Then

$$
\delta^{*}(B)_{v}={ }_{v} \operatorname{hom}_{R}\left(I_{B}, K_{B}\right)-{ }_{v} \operatorname{ext}_{B}^{1}\left(I_{B} / I_{B}^{2}, K_{B}\right)-\operatorname{dim}(\operatorname{im} \psi)_{-v} .
$$

Remark 32 . If $B$ is generically syzygetic, then ${ }_{v} \operatorname{Hom}_{B}\left(\mathrm{H}_{2}(R, B, B), K_{B}\right)=0$, and we get ${ }_{-v} \mathrm{H}_{\mathfrak{m}}^{n}\left(\mathrm{H}_{2}(R, B, B)\right)=0$ by duality and hence $\delta^{*}(B)_{v}=\delta(B)_{v}$, i.e. the "old and the new" definition of $\delta$ coincides.

Using (28) and ${ }_{v} \operatorname{Ext}_{B}^{i}\left(I_{B} / I_{B}^{2}, K_{B}\right) \simeq{ }_{-v} \mathrm{H}_{\mathfrak{m}}^{n-i}\left(I_{B} / I_{B}^{2}\right)^{\vee}$ and letting $h_{\mathfrak{m}}^{j}(-)_{v}=$ $\operatorname{dim}\left({ }_{v} \mathrm{H}_{\mathfrak{m}}^{j}(-)\right)$, one shows

$$
\delta^{*}(B)_{v}=h_{\mathfrak{m}}^{n+1}\left(S_{2} I_{B}\right)_{-v}-h_{\mathfrak{m}}^{n}\left(S_{2} I_{B}\right)_{-v}-h_{\mathfrak{m}}^{n+1}\left(I_{B}\right)_{-v} .
$$

Indeed splitting (28) into two short exact sequences we see that $h_{\mathfrak{m}}^{n-1}\left(I_{B} / I_{B}^{2}\right)_{-v}=$ $h_{\mathfrak{m}}^{n}\left(I_{B}^{2}\right)_{-v}$ and $h_{\mathfrak{m}}^{n}\left(I_{B} / I_{B}^{2}\right)_{-v}=h_{\mathfrak{m}}^{n+1}\left(I_{B}^{2}\right)_{-v}-h_{\mathfrak{m}}^{n+1}\left(I_{B}\right)_{-v}$. Moreover $h_{\mathfrak{m}}^{n+1}\left(I_{B}^{2}\right)_{-v}=$ $h_{\mathfrak{m}}^{n+1}\left(S_{2} I_{B}\right)_{-v}$ and $h_{\mathfrak{m}}^{n}\left(I_{B}^{2}\right)_{-v}=h_{\mathfrak{m}}^{n}\left(S_{2} I_{B}\right)_{-v}-\operatorname{dim}(\operatorname{im} \psi)_{-v}$; thus we get (29) from Definition 31 .

Proposition 33. Let $R$ be a finitely generated polynomial $k$-algebra with canonical module $R(e)$ (so $e=-n-c$ ), and let $B$ and $B^{\prime}$ be graded Cohen-Macaulay quotients, algebraically linked by a complete intersection $R \rightarrow D$ of dimension $n \geq 1$, and with minimal resolution

$$
0 \rightarrow R(-a) \rightarrow \ldots \rightarrow \bigoplus_{i=1}^{c} R\left(-c_{i}\right) \rightarrow I_{D} \rightarrow 0 .
$$

Let $\beta(B, D)_{v}:=\operatorname{dim} B_{v}+\sum_{i=1}^{c} \operatorname{dim}\left(I_{B / D}\right)_{v+c_{i}}$. Then $I_{B / D} \simeq K_{B^{\prime}}(-e-a)$ and

(i) $\operatorname{dim}\left(N_{B}\right)_{0}-\sum_{i=1}^{c} \operatorname{dim} B_{c_{i}}=\operatorname{dim}\left(N_{B^{\prime}}\right)_{0}-\sum_{i=1}^{c} \operatorname{dim} B_{c_{i}}^{\prime}$.

(ii) If $B$ and $B^{\prime}$ are generically Gorenstein (resp. Gorenstein in codimension $\leq 1)$, then ${ }_{v} \operatorname{Ext}_{B}^{i}\left(I_{B} / I_{B}^{2}, B\right)$ and ${ }_{v} \operatorname{Ext}_{B^{\prime}}^{i}\left(I_{B^{\prime}} / I_{B^{\prime}}^{2}, B^{\prime}\right)$ are isomorphic for $i=1$ (resp. $i=2$ ).

(iii) $\delta^{*}(B)_{v-2 e-2 a}=\beta\left(B^{\prime}, D\right)_{v-e-a}-\operatorname{dim}\left(K_{B^{\prime}}^{*}\right)_{v}$ for any $v$. Moreover, if $\beta\left(B^{\prime}, D\right)_{v-e-a}=0$ and $I_{B}$ is generically syzygetic, then

$$
v-2 e-2 a e^{2} t_{B}^{1}\left(I_{B} / I_{B}^{2}, K_{B}\right)=\operatorname{dim}\left(K_{B^{\prime}}^{*}\right)_{v} .
$$

(iv) $\operatorname{dim}\left(K_{B}^{*}\right)_{v}-\delta^{*}(B)_{-v}-\beta(B, D)_{v-e-a}=\operatorname{dim}\left(K_{B^{\prime}}^{*}\right)_{-v+2 e+2 a}-\delta^{*}\left(B^{\prime}\right)_{v-2 e-2 a}$ $-\beta\left(B^{\prime}, D\right)_{-v+e+a}$.

(v) If $I_{B}$ is generically syzygetic (resp. syzygetic in codimension $\leq 1$ ), then ${ }_{v} \operatorname{Ext}_{B}^{2}\left(I_{B} / I_{B}^{2}, K_{B}\right) \hookrightarrow{ }_{v+2 e+2 a} \operatorname{Ext}_{B^{\prime}}^{1}\left(S_{2}\left(K_{B^{\prime}}\right), K_{B^{\prime}}\right)$ is injective (resp. an isomorphism).

(vi) The conclusion of (ii) holds if $B$ is linked to $B^{\prime}$ in possibly several steps. Here the algebras of the intermediate steps need not necessarily be generically Gorenstein. 
Proof. (i) is proved in [27, Prop. 1.7.

(ii) This part, i.e. the liaison invariance of $I_{B} / I_{B}^{2} \otimes K_{B}$, is also known ([5], or [29], Ch. 6). Here the Gorenstein assumption is required for having ${ }_{v} \operatorname{Ext}_{B}^{i}\left(I_{B} / I_{B}^{2}, B\right) \simeq$ ${ }_{v} \operatorname{Ext}_{B}^{i}\left(I_{B} / I_{B}^{2} \otimes K_{B}, K_{B}\right)$. Note that the liaison invariance of $I_{B} / I_{B}^{2} \otimes K_{B}$ takes care of (vi) as well.

To prove (iii), recall that $K_{D} \simeq D(e+a)$ and hence $I_{B / D} \simeq \operatorname{Hom}_{D}(B, D) \simeq$ $K_{B^{\prime}}(-e-a)$, i.e. we get exact sequences

$$
\begin{gathered}
0 \rightarrow I_{D} \cdot I_{B} \rightarrow S_{2} I_{B} \rightarrow S_{2}\left(K_{B^{\prime}}\right)(-2 e-2 a) \rightarrow 0 \\
0 \rightarrow I_{D} \cdot I_{B} \rightarrow I_{D} \rightarrow I_{D} \otimes_{R} R / I_{B} \simeq I_{D} / I_{D}^{2} \otimes_{D} B \simeq \bigoplus_{i=1}^{c} B\left(-c_{i}\right) \rightarrow 0 ;
\end{gathered}
$$

cf. 38, Lemma 4.2.7, to see (30). Since $\beta\left(B^{\prime}, D\right)_{v}:=\operatorname{dim} B_{v}^{\prime}+\sum_{i=1}^{c} \operatorname{dim}\left(I_{B^{\prime} / D}\right)_{v+c_{i}}$ and $I_{B^{\prime} / D} \simeq K_{B}(-e-a)$, we get $\beta\left(B^{\prime}, D\right)_{v}=h_{\mathfrak{m}}^{n}\left(K_{B^{\prime}}\right)_{-v}+\sum_{i} h_{\mathfrak{m}}^{n}(B)_{e-v+a-c_{i}}$ by Gorenstein duality. Using (29) and (30), we have $\operatorname{dim}\left(K_{B^{\prime}}^{*}\right)_{v}=h_{\mathfrak{m}}^{n}\left(S_{2} K_{B^{\prime}}\right)_{-v}=$ $-\delta^{*}(B)_{-2 e+v-2 a}-h_{\mathfrak{m}}^{n+1}\left(I_{B}\right)_{2 e-v+2 a}+h_{\mathfrak{m}}^{n+1}\left(I_{D} I_{B}\right)_{2 e-v+2 a}$ while (31) and

$$
h_{\mathfrak{m}}^{n}\left(K_{B^{\prime}}\right)_{-v+e+a}=h_{\mathfrak{m}}^{n+1}\left(I_{D}\right)_{2 e-v+2 a}-h_{\mathfrak{m}}^{n+1}\left(I_{B}\right)_{2 e-v+2 a}
$$

show

$$
-h_{\mathfrak{m}}^{n+1}\left(I_{B}\right)_{2 e-v+2 a}+h_{\mathfrak{m}}^{n+1}\left(I_{D} I_{B}\right)_{2 e-v+2 a}=\beta\left(B^{\prime}, D\right)_{v-e-a} .
$$

Hence we have proved

$$
\operatorname{dim}\left(K_{B^{\prime}}^{*}\right)_{v}=-\delta^{*}(B)_{-2 e+v-2 a}+\beta\left(B^{\prime}, D\right)_{v-e-a} .
$$

Moreover if $\beta\left(B^{\prime}, D\right)_{v-e-a}=0$, then $\operatorname{dim} B_{v-e-a}^{\prime}=0$, i.e. $v-e-a<0$ and hence $H_{\mathfrak{m}}^{n+1}\left(I_{D}\right)_{2 e-v+2 a}=0$ by duality (e.g. if $\operatorname{dim} R>n+1$, then $h_{\mathfrak{m}}^{n+1}\left(I_{D}\right)_{2 e-v+2 a}=$ $h_{\mathfrak{m}}^{n}(D)_{2 e-v+2 a}=h_{\mathfrak{m}}^{n}\left(K_{D}\right)_{-v+e+a}=0$ by duality). Since $h_{\mathfrak{m}}^{n}\left(K_{B^{\prime}}\right)_{-v+e+a}=0$, it follows that $h_{\mathfrak{m}}^{n+1}\left(I_{D} I_{B}\right)_{2 e-v+2 a}=\beta\left(B^{\prime}, D\right)_{v-e-a}=0$ which easily leads to the final conclusion of (iii).

Note that (32) also shows $\operatorname{dim}\left(K_{B}^{*}\right)_{v}=-\delta^{*}\left(B^{\prime}\right)_{v-2 e-2 a}+\beta(B, D)_{v-e-a}$. Moreover if we replace $v$ by $-v+2 e+2 a$ in (32), we get (iv) by combining these formulas.

To prove (v), we use Gorenstein duality. Indeed

$$
{ }_{v} \operatorname{Ext}_{B}^{2}\left(I_{B} / I_{B}^{2}, K_{B}\right) \simeq{ }_{-v} \mathrm{H}_{\mathfrak{m}}^{n-2}\left(I_{B} / I_{B}^{2}\right)^{\vee} \simeq{ }_{-v} \mathrm{H}_{\mathfrak{m}}^{n-1}\left(I_{B}^{2}\right)^{\vee} \hookrightarrow{ }_{-v} \mathrm{H}_{\mathfrak{m}}^{n-1}\left(S_{2} I_{B}\right)^{\vee},
$$

where the final injection follows from Gorenstein duality and

$$
{ }_{v} \operatorname{Hom}_{B}\left(\mathrm{H}_{2}(R, B, B), K_{B}\right)=0
$$

because $I_{B}$ is generically syzygetic. Note that the injection is an isomorphism if $I_{B}$ is syzygetic in codimension $\leq 1$. Now combining (30) and (31) we get

$$
{ }_{-v} \mathrm{H}_{\mathfrak{m}}^{n-1}\left(S_{2} I_{B}\right)^{\vee} \simeq{ }_{-v} \mathrm{H}_{\mathfrak{m}}^{n-1}\left(S_{2}\left(K_{B^{\prime}}\right)(-2 e-2 a)\right)^{\vee} \simeq{ }_{v+2 e+2 a} \operatorname{Ext}_{B^{\prime}}^{1}\left(S_{2}\left(K_{B^{\prime}}\right), K_{B^{\prime}}\right),
$$

and we conclude easily.

Remark 34. With notations as in Proposition 33, allowing also $n=\operatorname{dim} B \geq 0$, we have

$$
\operatorname{dim}\left(N_{B}\right)_{v}-\sum_{i=1}^{c} \operatorname{dim} B_{v+c_{i}}=\operatorname{dim}\left(N_{B^{\prime}}\right)_{v}-\sum_{i=1}^{c} \operatorname{dim} B_{v+c_{i}}^{\prime} \text { for every integer } v .
$$

This follows from [27, Prop. 1.7, for $v=0$, and from [26], Cor. 2.12 and (2.19), or [29, Prop. 9.20, for $n \geq 2$ and every $v$. Since the arguments of the proof of [27, Prop. 1.7 hold for the linkage appearing in the non-graded deformation functors, 
we may use the proof to get the displayed dimension formula by showing that the isomorphism of their tangent spaces, $A_{D \rightarrow B}^{1} \simeq A_{D \rightarrow B^{\prime}}^{1}$ (whose degree zero parts are given by (10) ), is graded. This isomorphism is, however, concretely studied in 29], pp. 38-39, without using deformations. There, if we take the diagrams (6.11) and (6.12) without sheafification, we get a graded isomorphism $A_{D \rightarrow B}^{1} \simeq A_{D \rightarrow B^{\prime}}^{1}$ from which we get the displayed formula by e.g. the proof of [29], Prop.9.20.

Example 35. We now complete Example 17 for the remaining cases $(0 \leq s \leq$ 2 ) by verifying the assumptions of Proposition [13A), using liaison. Since we in Example 17 proved ${ }_{0} \operatorname{Ext}_{B}^{1}\left(I_{B} / I_{B}^{2}, B\right)=0$, it suffices by Remark 6r) to show ${ }_{0} \operatorname{Ext}_{B}^{2}\left(I_{B} / I_{B}^{2}, K_{B}(-s)\right)=0$. Now if we link $B$ to a $B^{\prime}$ via a complete intersection of three hypersurfaces, all of degree 2, the linked curve $C^{\prime}=\operatorname{Proj}\left(B^{\prime}\right)$ has degree $d^{\prime}=8-4=4$ and genus $g^{\prime}=0$, i.e. we see that $C$ and $C^{\prime}$ are essentially the same curve. Hence ${ }_{v} \operatorname{Ext}_{B^{\prime}}^{1}\left(S_{2}\left(K_{B^{\prime}}\right), K_{B^{\prime}}\right)=0$ for $v \geq 0$ since $B$ satisfies this vanishing. By Proposition 33 it follows that ${ }_{-s} \operatorname{Ext}_{B}^{2}\left(I_{B} / I_{B}^{2}, K_{B}\right) \hookrightarrow$ ${ }_{-s+2} \operatorname{Ext}_{B^{\prime}}^{1}\left(S_{2}\left(K_{B^{\prime}}\right), K_{B^{\prime}}\right)=0$ for $s \leq 2$, which precisely covers the cases of Example 17 which are not already analyzed. So, for $0 \leq s \leq 2$, Proposition [13A) applies! In Example 17 we saw that $\operatorname{dim}\left(K_{B}^{*}\right)_{s}=4 s+3$ for any $s \geq 0$. It remains to compute $\delta(B)_{-s}$. Since $\delta(B)_{v-2}=\beta\left(B^{\prime}, D\right)_{v-1}-\operatorname{dim}\left(K_{B^{\prime}}^{*}\right)_{v}$ and $\beta\left(B^{\prime}, D\right)_{v}=$ $\operatorname{dim} B_{v}^{\prime}+3 \operatorname{dim}\left(I_{B^{\prime} / D}\right)_{v+2}$ by Proposition 33, we get $\left(\delta(B)_{0}, \delta(B)_{-1}, \delta(B)_{-2}\right)$ to be equal to $(15,3,-3)$, and as previously

$$
\operatorname{dim}_{(A)} \operatorname{GradAlg}^{H}(R)=h^{0}\left(\mathcal{N}_{C}\right)+h^{0}\left(\theta_{C}(s)\right)-1-\delta(B)_{-s}=4 s+23-\delta(B)_{-s} .
$$

Hence $\operatorname{dim}_{(A)} \operatorname{GradAlg}^{H}(R)$ equals $(8,24,34)$ for $s=(0,1,2)$, respectively. Note that it is easy, for $s=2$, to see that $\left(K_{B}\right)_{0}=0$ and hence that ${ }_{-2} \operatorname{Hom}\left(I_{B} / I_{B}^{2}, K_{B}\right)$ $=0$ and ${ }_{-2} \operatorname{ext}_{B}^{1}\left(I_{B} / I_{B}^{2}, K_{B}\right)=-\delta(B)_{-2}=3$ by looking to the minimal resolution of $I_{B}$ (or use Proposition 33(iii)). By the interpretation of ${ }_{-s}$ ext $t_{B}^{1}\left(I_{B} / I_{B}^{2}, K_{B}\right)$ of Proposition $13 \mathrm{~A}$ ), we get that the codimension of the Gorenstein quotients given by (13) is 3 .

Example 36. Now we consider the Artinian reduction $A_{L}$ of Example 21 of socle degree $s+1$ to treat the remaining cases of interest $(0 \leq s \leq 2)$. Since $s \leq 2$ and $I_{B}$ has no linear generator, $s+1 \mathrm{H}_{2}\left(R_{L}, B_{L}, B_{L}\right) \hookrightarrow\left(S_{2} I_{B_{L}}\right)_{s+1}=0$. Looking to Example 21 and Remark 14 $\mathrm{b}$ ) we see that all assumptions of Proposition [13A) are satisfied. Now examining $\mathrm{H}^{i}\left(\theta_{C}(v)\right)$ of Example 17 for $v=-1$ a little further and invoking $\left(S_{2} K_{B}\right)_{1}=0$ we get ${ }_{-1} \operatorname{ext}_{B}^{1}\left(S_{2}\left(K_{B}\right), K_{B}\right)=1$ in addition to ${ }_{v} \operatorname{Ext}_{B}^{1}\left(S_{2}\left(K_{B}\right), K_{B}\right)=0$ for $v \geq 0$. By Lemma 19)(i) we get $\operatorname{dim}\left(K_{B_{L}}^{*}\right)_{v}=4$ for $v \geq 1$. Moreover, if we apply $\operatorname{Hom}_{B}\left(I_{B} / I_{B}^{2},-\right)$ to the exact sequence $0 \rightarrow K_{B} \rightarrow$ $K_{B}(1) \rightarrow K_{B_{L}} \rightarrow 0$, we get

$$
\delta\left(B_{L}\right)_{v-1}=\delta(B)_{v}-\delta(B)_{v-1}+{ }_{v} \operatorname{ext}_{B}^{2}\left(I_{B} / I_{B}^{2}, K_{B}\right)-{ }_{v-1} \operatorname{ext}_{B}^{2}\left(I_{B} / I_{B}^{2}, K_{B}\right) .
$$

Since ${ }_{-3} \operatorname{ext}_{B}^{2}\left(I_{B} / I_{B}^{2}, K_{B}\right)={ }_{-1} \operatorname{ext}_{B^{\prime}}^{1}\left(S_{2}\left(K_{B^{\prime}}\right), K_{B^{\prime}}\right)=1$ and ${ }_{-s} \operatorname{Ext}_{B}^{2}\left(I_{B} / I_{B}^{2}, K_{B}\right)$ $=0$ for $s \leq 2$, we get that $\left(\delta\left(B_{L}\right)_{-1}, \delta\left(B_{L}\right)_{-2}, \delta\left(B_{L}\right)_{-3}\right)$ is equal to $(12,6,-4)$ from the values of $\delta(B)_{v}$ in Example 35. It follows that $A_{L}$ is an unobstructed Artinian Gorenstein algebra for $0 \leq s \leq 2$, i.e. PGor $\left(H_{L}\right)$ is smooth at $\left(A_{L}\right)$ by Theorem 11 and

$\operatorname{dim}_{\left(A_{L}\right)} \operatorname{PGor}\left(H_{L}\right)=\operatorname{dim}\left(N_{B_{L}}\right)_{0}+\operatorname{dim}\left(K_{B_{L}}^{*}\right)_{s+1}-1-\delta\left(B_{L}\right)_{-s-1}=15-\delta\left(B_{L}\right)_{-s-1}$. Moreover, for $s=2$, we see that $-\delta\left(B_{L}\right)_{-3}={ }_{-3} e x t_{B_{L}}^{1}\left(I_{B_{L}} / I_{B_{L}}^{2}, K_{B_{L}}\right)=4$ is the codimension of the stratum of Gorenstein quotients given by (13) around $\left(A_{L}\right)$. 
Finally we remark that the conclusions (about $A_{L}$ ) also hold for any Artinian Gorenstein quotient $A$, well-defined by a twist of the canonical module of $B$, with the same graded Betti numbers as $A_{L}$. In this case we can compute the numbers $\delta(B)_{v}$ of a one-dimensional $B$ as we did in Example 35 by essentially the same linkage argument.

Mainly using Proposition 33 twice (for (i) below we utilize (33)), we get

Corollary 37. Let $B$ and $B^{\prime}$ be Cohen-Macaulay quotients of $R$, algebraically linked by a complete intersection $D$ of type $\left(c_{1}, c_{2}, . ., c_{c}\right)$, let $B^{\prime}$ and $B^{\prime \prime}$ be algebraically linked by a complete intersection $D^{\prime}$ of type $\left(c_{1}^{\prime}, c_{2}^{\prime}, . ., c_{c}^{\prime}\right)$, and let $a=\sum c_{i}$, $a^{\prime}=\sum c_{i}^{\prime}$ and $\operatorname{dim} B>0$. Then:

(i) $v+2 a^{\prime} \operatorname{Ext}_{B^{\prime \prime}}^{1}\left(S_{2}\left(K_{B^{\prime \prime}}\right), K_{B^{\prime \prime}}\right) \simeq v+2 a \operatorname{Ext}_{B}^{1}\left(S_{2}\left(K_{B}\right), K_{B}\right)$ for any $v$.

(ii) $v-2 a^{\prime} \operatorname{Ext}_{B^{\prime \prime}}^{2}\left(I_{B^{\prime \prime}} / I_{B^{\prime \prime}}^{2}, K_{B^{\prime \prime}}\right) \simeq{ }_{v-2 a} \operatorname{Ext}_{B}^{2}\left(I_{B} / I_{B}^{2}, K_{B}\right)$ provided $I_{B}$ and $I_{B^{\prime \prime}}$ are syzygetic in codimension $\leq 1$.

(iii) $\delta^{*}\left(B^{\prime \prime}\right)_{v-2 e-2 a^{\prime}}=\delta^{*}(B)_{v-2 e-2 a}-\beta\left(B^{\prime}, D\right)_{v-e-a}+\beta\left(B^{\prime}, D^{\prime}\right)_{v-e-a^{\prime}}$.

(iv) $\operatorname{dim}\left(K_{B^{\prime \prime}}^{*}\right)_{v+2 e+2 a^{\prime}}=\operatorname{dim}\left(K_{B}^{*}\right)_{v+2 e+2 a}-\beta\left(B^{\prime}, D\right)_{v+e+a}+\beta\left(B^{\prime \prime}, D^{\prime}\right)_{v+e+a^{\prime}}$ or equivalently, $\operatorname{dim}\left(K_{B^{\prime \prime}}^{*}\right)_{v+2 e+2 a^{\prime}}=\operatorname{dim}\left(K_{B}^{*}\right)_{v+2 e+2 a}+\operatorname{dim} B_{v+e+a^{\prime}}^{\prime \prime}-$ $\operatorname{dim} B_{v+e+a}+\sum_{i=1}^{c}\left(\operatorname{dim}\left(K_{B^{\prime}}\right)_{v+c_{i}^{\prime}}-\operatorname{dim}\left(K_{B^{\prime}}\right)_{v+c_{i}}\right)$.

We include one example of biliaison starting with the smooth rational curve of Example 17. Of course starting with a licci curve, it is immediate to verify the assumptions of Proposition 13 (see Theorem 16). However, some part of the argument below is still needed to find $\operatorname{dim}\left(K_{B}^{*}\right)_{s}-\delta^{*}(B)_{-s}$ and to see how small we can let the number $s$ in Theorem $16 \mathrm{~B})$ be.

Example 38. If we link the homogeneous coordinate ring $B$ of the smooth rational quartic curve of Example 17 having minimal resolution

$$
0 \rightarrow R(-4)^{3} \rightarrow R(-3)^{8} \rightarrow R(-2)^{6} \rightarrow R \rightarrow B \rightarrow 0
$$

to a $B^{\prime}$ using a complete intersection of type $(2,2,3)$ and further link $B^{\prime}$ to a $B^{\prime \prime}$ via a complete intersection of type $(2,3,4)$ (using the same hypersurface of degree 2 and 3 as in the first link), one may, notably due to $I_{B^{\prime} / D} \simeq K_{B}(-2)$ and the mapping cone construction used twice, see that the minimal resolution of $B^{\prime \prime}$ is

$$
0 \rightarrow R(-7) \oplus R(-6)^{3} \rightarrow R(-5)^{10} \rightarrow R(-4)^{5} \oplus R(-3) \oplus R(-2) \rightarrow R \rightarrow B^{\prime \prime} \rightarrow 0 .
$$

If $A$ is defined by a regular section of $\left(K_{B^{\prime \prime}}^{*}\right)_{s}$, we can easily find the minimal resolution of $A$ and its Hilbert function by using (13). For instance $\operatorname{dim} A_{v}=$ $16 v-19$ for $3 \leq v \leq s-3$ and $\operatorname{dim} A_{v}=16 s-38$ for $v>s$. The $h$-vector of $A$ is in fact

$$
(1,4,8,8,4,1),(1,4,9,14,9,4,1),(1,4,9,15,15,9,4,1),(1,4,9,15,16,15,9,4,1)
$$

for $s=4,5,6,7$ and $(1,4,9,15,16, \ldots, 16,15,9,4,1)$ for $s \geq 8$, respectively. Let us suppose $s>3$ since otherwise $\operatorname{Proj}(A)$ is degenerate. To use Proposition 13, we may argue as in Example 17 to see that $\delta\left(B^{\prime \prime}\right)_{-s}=0$ for $s>7$ by Remark 14a), and that $\mathrm{H}^{1}\left(\theta_{C^{\prime \prime}}(v)\right)=0$ and hence $\operatorname{dim}\left(K_{B^{\prime \prime}}^{*}\right)_{v}=h^{0}\left(\theta_{C^{\prime \prime}}(v)\right)=16 v-57$ for $v \geq 5$, because $C^{\prime \prime}:=\operatorname{Proj}\left(B^{\prime \prime}\right)$ is smooth curve of degree $d^{\prime \prime}=16$ and genus $g^{\prime \prime}=20$. Corollary 37 leads, however, to more precise information. Indeed it is rather straightforward to combine the corollary and the computations of Example 17 and Example 35 to see that the conclusions of Theorem [16 hold for $s \geq 4$, that $\operatorname{dim}\left(K_{B^{\prime \prime}}^{*}\right)_{4}=8$, and that $\left(\delta\left(B^{\prime \prime}\right)_{-4}, \delta\left(B^{\prime \prime}\right)_{-5}, \delta\left(B^{\prime \prime}\right)_{-6}\right)$ is equal to $(7,-6,-6)$ and $\delta\left(B^{\prime \prime}\right)_{-s}=0$ for $s>6$. 
Finally patiently using Proposition $33(\mathrm{i})$ several times, one shows $\operatorname{dim}\left(N_{B^{\prime \prime}}\right)_{0}=$ 62. (It is, however, faster to show ${ }_{0} \operatorname{Ext}_{B^{\prime \prime}}^{i}\left(I_{B^{\prime \prime}} / I_{B^{\prime \prime}}^{2}, B^{\prime \prime}\right)=0$ for $i=1$ and 2 by Proposition 33(ii), using the computations of Example 17 and (15), and then use (15) once more to see $h^{1}\left(\mathcal{N}_{C^{\prime \prime}}\right)=1$ and hence $\operatorname{dim}\left(N_{B^{\prime \prime}}\right)_{0}=5 d^{\prime \prime}+1-g^{\prime \prime}+$ $\left.h^{1}\left(\mathcal{N}_{C^{\prime \prime}}\right)=62\right)$. Putting this together we have by Proposition 13 that $A$ is an unobstructed Gorenstein algebra for $s \geq 4$,

$$
\operatorname{dim}_{(A)} \operatorname{GradAlg}^{H}(R)=\operatorname{dim}\left(N_{B^{\prime \prime}}\right)_{0}+\operatorname{dim}\left(K_{B^{\prime \prime}}^{*}\right)_{s}-1-\delta\left(B^{\prime \prime}\right)_{-s}=16 s+4
$$

for $s \geq 7, \operatorname{dim}_{(A)} \operatorname{GradAlg}^{H}(R)=16 s+10$ for $5 \leq s \leq 6$ and $\operatorname{dim}_{(A)} \operatorname{GradAlg}^{H}(R)=$ 62 for $s=4$. By the interpretation of ${ }_{-s} e x t_{B^{\prime \prime}}^{1}\left(I_{B^{\prime \prime}} / I_{B^{\prime \prime}}^{2}, K_{B^{\prime \prime}}\right)$ of Proposition 13 , we get by Proposition [33(iii) that the codimension of the stratum of Gorenstein quotients given by (13) is 6 for $5 \leq s \leq 6$.

We may treat the Artinian reduction of $A \simeq B^{\prime \prime} / K_{B^{\prime \prime}}(-s)$ in Example 38 satisfactorily by remarking that ${ }_{s+1} \mathrm{H}_{2}\left(R_{L}, B_{L}^{\prime \prime}, B_{L}^{\prime \prime}\right)=0$ for $4 \leq s \leq 6$ (since one may show $\left(S_{2} I_{B_{L}^{\prime \prime}}\right)_{s+1} \simeq\left(I_{B_{L}^{\prime \prime}}^{2}\right)_{s+1}$ by using that the generators of $I_{B_{L}^{\prime \prime}}$ of degree 2 and 3 form a regular sequence), and that $B_{L}^{\prime \prime}$ is unobstructed by Lemma 19)(iii) for any $s>3$. For the latter, we use the liaison invariance of ${ }_{v} \operatorname{Ext}_{B}^{1}\left(I_{B} / I_{B}^{2}, B\right)$ and the fact that $\mathrm{H}^{1}\left(\mathcal{N}_{C}(v)\right)=0$ for $v \geq-1$ for a smooth rational curve $C$. Instead of working out these details, we will finish this subsection by considering two general classes of examples where $B$ is licci. We concentrate on licci codimension 3 quotients, leading to unobstructed Gorenstein codimension 4 quotients by Theorem [16, In one of the cases the use of Proposition 33 is quite effective.

Example 39. Let $B$ be a Gorenstein quotient of $R$ of codimension 3 given by

$$
0 \rightarrow R(-f) \simeq F_{3} \rightarrow F_{2} \rightarrow F_{1} \rightarrow I_{B} \rightarrow 0
$$

(as $A$ in Example 28). In this case $e=-n-3$ and $K_{B} \simeq B(-f+n+3)$ and since $B$ is licci ([38]) and therefore satisfies ${ }_{v} \operatorname{Ext}_{B}^{1}\left(I_{B} / I_{B}^{2}, B\right)=0$ for any $v$ by Proposition 33, we get $\delta(B)_{-s}=\operatorname{dim}\left(N_{B}\right)_{-(f+s-n-3)}$. If $A$ is defined by a section of $\left(K_{B}^{*}\right)_{s}$ as in Theorem 16, then $A$ is Gorenstein and unobstructed and

$$
\operatorname{dim}_{(A)} \operatorname{GradAlg}^{H_{A}}(R)=\operatorname{dim}\left(N_{B}\right)_{0}+\operatorname{dim} B_{f+s-n-3}-1-\operatorname{dim}\left(N_{B}\right)_{-f-s+n+3},
$$

where $\operatorname{dim}\left(N_{B}\right)_{v}$ is given by [28], Thm. 2.6, i.e. by modifying (20) to

$$
\begin{gathered}
\operatorname{dim}\left(N_{B}\right)_{v}=\operatorname{dim}\left(\bigwedge^{2} F_{1}\right)_{f+v}-\operatorname{dim}\left(F_{1} \otimes F_{2}\right)_{f+v}+\operatorname{dim}\left(S_{2}\left(F_{2}\right)\right)_{f+v} \\
+\operatorname{dim}\left(F_{1}\right)_{v}-\operatorname{dim}\left(F_{2}\right)_{v} .
\end{gathered}
$$

Example 40. Let $B$ be an almost complete intersection of $R$ of codimension 3 with a minimal resolution

$$
0 \rightarrow G_{3} \rightarrow G_{2} \rightarrow G_{1}=\bigoplus_{i=1}^{3} R\left(-c_{i}\right) \oplus R(-h) \rightarrow I_{B} \rightarrow 0
$$

and let $B^{\prime}$ be the Gorenstein quotient obtained by linking $B$ to $B^{\prime}$ via a complete intersection whose minimal generators (of degree $c_{i}$ ) corresponds to three of those of $I_{B}$. A standard mapping cone construction and duality yields

$$
0 \rightarrow R(h-a) \rightarrow G_{2}^{*}(-a) \rightarrow G_{3}^{*}(-a) \oplus\left(\bigoplus_{i=1}^{3} R\left(-c_{i}\right)\right) \rightarrow I_{B^{\prime}} \rightarrow 0
$$


and we see that $K_{B^{\prime}} \simeq B^{\prime}(a-h-n-3)$, where $a=\sum c_{i}$. If $A$ is defined by the zero locus of a section of $\left(K_{B}^{*}\right)_{s}$ as in Theorem 16, then $A$ is Gorenstein and unobstructed. Invoking Proposition 33 and $\operatorname{dim}\left(K_{B^{\prime}}^{*}\right)_{-s+2 a-2 n-6}-\delta\left(B^{\prime}\right)_{s-2 a+2 n+6}=$ $\operatorname{dim} B_{-s+h+a-n-3}^{\prime}-\operatorname{dim}\left(N_{B^{\prime}}\right)_{s-h-a+n+3}$ we get

$$
\begin{aligned}
\operatorname{dim}_{(A)} \operatorname{GradAlg}^{H_{A}}(R)= & \operatorname{dim}\left(N_{B^{\prime}}\right)_{0}+\operatorname{dim} B_{-s+h+a-n-3}^{\prime} \\
& -\operatorname{dim}\left(N_{B^{\prime}}\right)_{s-h-a+n+3}-1+\gamma,
\end{aligned}
$$

where

$$
\begin{aligned}
\gamma:=\sum_{i=1}^{3} & \operatorname{dim} B_{c_{i}}-\operatorname{dim} B_{c_{i}}^{\prime}+\operatorname{dim}\left(I_{B / D}\right)_{s-a+n+3+c_{i}} \\
& \left.\quad-\operatorname{dim}\left(I_{B^{\prime} / D}\right)_{-s+a-n-3+c_{i}}\right)+\operatorname{dim} B_{s-a+n+3}-\operatorname{dim} B_{-s-n-3+a}^{\prime} .
\end{aligned}
$$

Using $I_{B / D} \simeq K_{B^{\prime}}(n+3-a) \simeq B^{\prime}(-h)$, we easily express $\gamma$ in terms of the Hilbert function of $B^{\prime}$ (or of $B$ ) and $D$. Moreover $\operatorname{dim}\left(N_{B^{\prime}}\right)_{v}$ is given by the final formula of Example 39. See Example 2 for a more explicit example.

\section{REFERENCES}

[1] M. André. Métode Simpliciale en Algèbre Homologique et Algèbre Commutative. Lecture Notes in Math., no. 32. Springer-Verlag, Berlin, New York, 1967. MR0214644 (35:5493)

[2] L. Avramov and J. Herzog. The Koszul algebra of a codimension 2 embedding. Math. Z. 175 (1980), 249-260. MR0602637 (82g:13011)

[3] M. Boij. Components of the space parametrizing graded Gorenstein Artin algebras with a given Hilbert function. Pacific J. of Math. 187 no. 1 (1999), 1-11. MR.1674301 (2000j:14006)

[4] M. Boij. Gorenstein Artin algebras and points in projective space. Bull. London Math. Soc. 31, no. 1 (1999), 11-16. MR1651033 (99i:13033)

[5] R. Buchweitz and B. Ulrich. Homological properties which are invariant under linkage. Preprint 1983.

[6] A. Conca and G. Valla. Hilbert function of powers of ideals of low codimension. Math. Z. 230 no. 4 (1999), 753-784. MR1686559 (2000e:13022)

[7] D. Eisenbud. Commutative algebra. With a view toward algebraic geometry. Graduate Texts in Math., Vol. 150, Springer-Verlag, New York, 1995. MR1322960 (97a:13001)

[8] A.V. Geramita, T. Harima and Y.S. Shin. Extremal Point Sets and Gorenstein Ideals. Adv. Math. 152 (2000), 78-119. MR.1762121(2001d:13015)

[9] A.V. Geramita, H.J. Ko and Y.S. Shin. The Hilbert function and the minimal free resolution of some Gorenstein ideals of codimension 4, Communications in Algebra 26 (1998), 42854307. MR1661265 (99m:13032)

[10] A.V. Geramita and J. Migliore. Reduced Gorenstein codimension three subschemes of projective space. Proc. Amer. Math. Soc. 125 (1997), 943-950. MR1403128 (97h:14068)

[11] A. Grothendieck. Les schemas des Hilbert. Séminaire Bourbaki, exp. 221 (1960). MR.1611822

[12] A. Grothendieck. Cohomologie Locale des Faisceaux Cohérents et Théorémes de Lefschetz Locaux et Globaux. Augmenté d'un exposé par M. Raynaud. (SGA 2). Advanced Studies in Pure Mathematics, Vol. 2. North-Holland, Amsterdam (1968). MR0476737 (57:16294)

[13] A. Grothendieck. Eléments de la géométrie algebriques IV. Etude locale des schémas et des morphismes de schémas Publ. Math. I.H.E.S. 32 (1967). MR0238860 (39:220)

[14] A. Grothendieck. Groupes de Monodromie en Géométrie Algébrique (SGA 7). Lecture Notes in Math., Vol. 288 (with M. Raynaud and D.S. Rim) (1972) and Vol. 340 (with P. Deligne and N. Katz) (1973) Springer-Verlag, Heidelberg. MR0354656 (50:7134) MR0354657 (50:7135)

[15] T. Harima. Characterization of Hilbert Functions of Gorenstein Artin algebras with the weak Stanley property. Proc. Amer. Math. Soc. 123 (1995), 3631-3638. MR1307527(96b:13014)

[16] R. Hartshorne. Local Cohomology. Lectures Notes in Math., Vol. 41, Springer-Verlag, New York, 1967. MR0224620 (37:219)

[17] R. Hartshorne. Connectedness of the Hilbert Scheme. Publ. Math. I.H.E.S. 29 (1966), 5-48. MR0213368 (35:4232) 
[18] J. Herzog. Deformationen von Cohen-Macaulay Algebren. J. Reine Angew. Math. 318 (1980), 83-105. MR0579384 (81m:13012)

[19] M. Haiman and B. Sturmfels. Multigraded Hilbert Schemes. J. Algebraic Geom. 13 no. 4 (2004), 725-769. MR2073194 (2005d:14006)

[20] C. Huneke. Linkage and the Koszul homology of ideals. Amer. J. Math. 104 no. 5 (1982), 1043-1062. MR0675309 (84f:13019)

[21] A. Iarrobino and V. Kanev. Power sums, Gorenstein Algebras and Determinantal Loci. Lectures Notes in Math., Vol. 1721, Springer-Verlag, New York, 1999. MR1735271|(2001d:14056)

[22] A. Iarrobino and H. Srinivasan. Some Gorenstein Artin algebras of embedding dimension four, I: components of $\operatorname{PGor}(H)$ for $H=(1,4,7, \ldots, 1)$. Preprint 2002.

[23] T. de Jong and D. van Straten. Deformations of normalization of hypersurfaces. Math. Ann. 288 (1990), 527-547. MR1079877 (92d:32050)

[24] J. O. Kleppe. Deformations of graded algebras. Math. Scand. 45 (1979), 205-231. MR0580600 $(82 \mathrm{j}: 13017)$

[25] J. O. Kleppe. Non-reduced components of the Hilbert scheme of smooth space curves, in "Space Curves, Proceedings (Rocca di Papa, 1985)" Lectures Notes in Math., Vol. 1266, Springer-Verlag, New York, 1987. MR0908714 (89a:14010)

[26] J. O. Kleppe. Liaison of families of subschemes in $\mathbb{P}^{n}$, in "Algebraic Curves and Projective Geometry, Proceedings (Trento, 1988)" Lectures Notes in Math., Vol. 1389, Springer-Verlag, New York, 1989. MR1023396 (90m:14011)

[27] J. O. Kleppe. The smoothness and the dimension of PGor $(H)$ and of other strata of the punctual Hilbert scheme. J. Algebra, 200 no. 2 (1998), 606-628. MR1610672 (99k:14007)

[28] J. O. Kleppe and R. Miro-Roig. The dimension of the Hilbert scheme of Gorenstein codimension 3 subschemes. J. Pure Appl. Algebra 127 no. 1 (1998), 73-82. MR1609504 (99a:14002)

[29] J.O. Kleppe, J. Migliore, R. Miro-Roig, U. Nagel and C. Peterson. Gorenstein Liaison, Complete Intersection Liaison Invariants and Unobstructedness. Mem. Amer. Math. Soc. 154 no. 732 (2001). MR1848976 (2002e:14083)

[30] J. O. Kleppe and C. Peterson. Gorenstein algebras and maximal Cohen-Macaulay modules. J. Algebra 238 (2001), 776-800. MR1823784 (2002e:13029)

[31] A. Laudal. Formal Moduli of Algebraic Structures. Lectures Notes in Math., Vol. 754, Springer-Verlag, New York, 1979. MR0551624 (82h:14009)

[32] A. Laudal. A generalized trisecant lemma, in Proceedings, Tromsø, 1977, Lectures Notes in Math., Vol. 687, Springer-Verlag, New York, 1978. MR0527232 (81f:14019)

[33] M. Martin-Deschamps and D. Perrin. Sur la classification des courbes gauches, I. Astérisque, 184-185 (1990). MR:1073438 (91h:14039)

[34] J. Migliore. Introduction to liaison theory and deficiency modules. Progress in Math., Vol. 165, Birkhäuser Boston, Inc., Boston, MA, 1998. MR1712469 (2000g:14058)

[35] J. Migliore and U. Nagel. Reduced arithmetically Gorenstein schemes and simplicial polytopes with maximal Betti numbers. Adv. Math. 180 (2003), 1-63. MR.2019214 (2004k:14082)

[36] J. Migliore and C. Peterson. A construction of codimension three arithmetically Gorenstein subschemes of projective space. Trans. Amer. Math. Soc. 349 no. 9 (1997), 3803-3821. MR1432204 (98d:14060)

[37] D. Mumford. Lectures on Curves on an Algebraic Surface. Annals of Math. Studies, Vol. 59, Princeton Univ. Press, 1966. MR0209285 (35:187)

[38] W. Vasconcelos. Arithmetic of Blowing up Algebras. London Mathematical Society. Lecture Note Series, Vol. 195, Cambridge University Press, 1994. MR:1275840 (95g:13005)

[39] B. Ulrich. Sums of linked ideals. Trans. Amer. Math. Soc. 318 (1990), 1-42. MR0964902 (90f:13012)

Faculty of Engineering, Oslo University College, Postboks 4, St. Olavs plass, N0130 OSLO, NORWAY

E-mail address: JanOddvar.Kleppe@iu.hio.no 